University of Tennessee Health Science Center UTHSC Digital Commons

\title{
$5-2011$
}

\section{Pten Function in Postnatal Brain Development and Tumorigenesis}

Guo Zhu

University of Tennessee Health Science Center

Follow this and additional works at: https://dc.uthsc.edu/dissertations

Part of the Medicine and Health Sciences Commons

\section{Recommended Citation}

Zhu, Guo, "Pten Function in Postnatal Brain Development and Tumorigenesis" (2011). Theses and Dissertations (ETD). Paper 325. http://dx.doi.org/10.21007/etd.cghs.2011.0383.

This Dissertation is brought to you for free and open access by the College of Graduate Health Sciences at UTHSC Digital Commons. It has been accepted for inclusion in Theses and Dissertations (ETD) by an authorized administrator of UTHSC Digital Commons. For more information, please contact jwelch30@uthsc.edu. 


\title{
Pten Function in Postnatal Brain Development and Tumorigenesis
}

\begin{abstract}
PTEN (Phosphatase and Tensin homolog deleted on chromosome 10) is a tumor suppressor gene that is commonly mutated in multiple types of human cancers including endometrial, prostate, breast cancers and glioblastoma multiforme (GBM). PTEN functions as a phospholipid phosphatase antagonizing phosphorylation by phosphatidylinositol-3-OH kinases (PI3Ks) and thus serves as a major negative regulator of the PI3K pathway. Homozygous Pten knock-out mice die during embryogenesis. Conditional Pten knock-out in the nervous system showed that Pten negatively regulates self-renewal and proliferation of neural stem cells, negatively controls neuronal size, is required for proper neuronal positioning and is involved in the development and maintenance of normal function for oligodendrocytes and astrocytes.
\end{abstract}

Neuronal precursors (neuroblasts) are generated every day throughout life in the subventricular zone (SVZ) and migrate through the rostral migratory stream (RMS) to the olfactory bulb where they differentiate into mature interneurons. The signaling pathways instructing when and where these tangentially migrating neuroblasts stop are largely unknown. In this study, we found that the PI3K-Akt-mTorc1 pathway is selectively inactivated in the migrating neuroblasts in the wild-type subventricular zone and rostral migratory stream and activated when they reach the olfactory bulb. Postnatal deletion of Pten in the SVZ resulted in aberrant activation of the PI3K-Akt-mTorc1 pathway and enlarged SVZ and RMS. This was caused by premature termination of migration and differentiation of neuroblasts, and could be rescued by inhibition of mTorc1. Although this is consistent with previous studies in lower organisms showing that Pten was required for directional migration, live imaging of acute brain slices showed that the migrating Pten-deficient neuroblasts were not defective in speed and showed appropriate directional movement. Therefore, the apparent "migration defect" in Pten-deficient neurons was secondary to ectopic differentiation rather than an intrinsic defect in directional migration.

Inactivation of Pten in the neural stem/progenitor cells at birth also revealed a novel perivascular proliferative niche in the cerebellum without full-blown tumor formation. Co-deletion of Pten and Trp53, two commonly mutated tumor suppressor genes in human brain tumors, could synergize in generating high-penetrant medulloblastoma with a growth pattern centering around blood vessels and extensive neuronal differentiation. The Pten and Trp53 double knock-out medulloblastomas spontaneously lost chromosome 7, 13 and 16 and had mutations in the gene Ptch1. Therefore the PI3K and Shh pathways cooperate in generating medulloblastomas.

Our results show Pten is required for appropriate positioning of neuronal differentiation and preventing ectopic neural stem/progenitor proliferation and medulloblastoma genesis.

\author{
Document Type \\ Dissertation \\ Degree Name \\ Doctor of Philosophy $(\mathrm{PhD})$ \\ Program \\ Biomedical Sciences \\ Research Advisor \\ Suzanne J. Baker, Ph.D.
}




\section{Keywords}

PTEN, subventricular zone, cerebellum, medulloblastoma

\section{Subject Categories}

Medicine and Health Sciences

\section{Comments}

Two year embargo expired May 2013 
PTEN FUNCTION IN POSTNATAL BRAIN DEVELOPMENT AND

TUMORIGENESIS

\author{
A Dissertation \\ Presented for \\ The Graduate Studies Council \\ The University of Tennessee \\ Health Science Center
}

In Partial Fulfillment

Of the Requirements for the Degree

Doctor of Philosophy

From The University of Tennessee

By

Guo Zhu

May 2011 
Copyright $(92011$ by Guo Zhu. All rights reserved. 


\section{DEDICATION}

To my wife Lingli Zhang To my father Ya-Zong Zhu To my mother Guo-E Li To my brother Ye Zhu 


\section{ACKNOWLEDGEMENTS}

First and foremost, I would like to thank my mentor Dr. Suzanne Baker for her guidance, support and encouragement during all the years of my graduate school and for providing the opportunity to work on this project. I admire Dr. Baker's elegance, precision and conciseness on science, her love for student and the amount of time spent on student education and her great balance between career and family. I have been feeling lucky to be able to study and work in the Baker laboratory, a comfortable, supporting and encouraging family that had made my transition and journey to a new country enjoyable and less dreadful. I thank Dr. Lionel Chow for making the Nestin$C r e E R^{T 2}$ transgenic mice and for teaching me everything including laboratory techniques and all the knowledge when I just joined the laboratory. I thank Jovan Mitchell and Kristen Cox for genotyping mice and thank all other Baker lab members Junyuan Zhang, Xiaoyan Zhu, Dr. Enrique Torchia, Dr. Melissa Fraser, Dr. Nader Chalhoub, Dr. Raelene Endersby, Dr. Barbara Paugh, Dr. Sherri Rankin, Dr. Claudia Miller and Dr. Troy McEachron for their helps, suggestions and discussions.

I am grateful for the help I got from Dr. David Solecki for brain slice preparation and migration analysis, Drs. Ildar Bayazitov and Stanislav Zakharenko for two-photon microscopy imaging, Dr. Yiai Tong in Dr. Richard Gilbertson's lab for in vivo electroporation. Without the teamwork from all these people, the ex vivo migration analysis would have been mission impossible.

I would also like to thank Dr. David Ellison for his pathological reviews on the brain tumors, the St. Jude Transgenic Core Facility for technical expertise in generating Nestin-CreER ${ }^{T 2}$ mice, the St. Jude Cell and Tissue Imaging Shared Resource for confocal microscopy and the St. Jude Cancer Center Core Cytogenetic Laboratory for FISH analysis. I thank Tak W. Mak (University of Toronto) for providing Pten loxP mice and Pierre Chambon (IGBMC) for Nestin-CreER ${ }^{T 2}$ cDNA.

My gratitude also extends to my committee members Drs. James Morgan, Michael Dyer, Gerard Zambetti, Lawrence Pfeffer and Seema Khurana. I appreciate their time for serving on my committee and their insightful suggestions. 


\begin{abstract}
PTEN (Phosphatase and Tensin homolog deleted on chromosome 10) is a tumor suppressor gene that is commonly mutated in multiple types of human cancers including endometrial, prostate, breast cancers and glioblastoma multiforme (GBM). PTEN functions as a phospholipid phosphatase antagonizing phosphorylation by phosphatidylinositol-3-OH kinases (PI3Ks) and thus serves as a major negative regulator of the PI3K pathway. Homozygous Pten knock-out mice die during embryogenesis. Conditional Pten knock-out in the nervous system showed that Pten negatively regulates self-renewal and proliferation of neural stem cells, negatively controls neuronal size, is required for proper neuronal positioning and is involved in the development and maintenance of normal function for oligodendrocytes and astrocytes.
\end{abstract}

Neuronal precursors (neuroblasts) are generated every day throughout life in the subventricular zone (SVZ) and migrate through the rostral migratory stream (RMS) to the olfactory bulb where they differentiate into mature interneurons. The signaling pathways instructing when and where these tangentially migrating neuroblasts stop are largely unknown. In this study, we found that the PI3K-Akt-mTorc1 pathway is selectively inactivated in the migrating neuroblasts in the wild-type subventricular zone and rostral migratory stream and activated when they reach the olfactory bulb. Postnatal deletion of Pten in the SVZ resulted in aberrant activation of the PI3K-Akt-mTorc1 pathway and enlarged SVZ and RMS. This was caused by premature termination of migration and differentiation of neuroblasts, and could be rescued by inhibition of mTorc1. Although this is consistent with previous studies in lower organisms showing that Pten was required for directional migration, live imaging of acute brain slices showed that the migrating Pten-deficient neuroblasts were not defective in speed and showed appropriate directional movement. Therefore, the apparent "migration defect" in Pten-deficient neurons was secondary to ectopic differentiation rather than an intrinsic defect in directional migration.

Inactivation of Pten in the neural stem/progenitor cells at birth also revealed a novel perivascular proliferative niche in the cerebellum without full-blown tumor formation. Co-deletion of Pten and Trp53, two commonly mutated tumor suppressor genes in human brain tumors, could synergize in generating high-penetrant medulloblastoma with a growth pattern centering around blood vessels and extensive neuronal differentiation. The Pten and Trp53 double knock-out medulloblastomas spontaneously lost chromosome 7,13 and 16 and had mutations in the gene Ptch1. Therefore the PI3K and Shh pathways cooperate in generating medulloblastomas.

Our results show Pten is required for appropriate positioning of neuronal differentiation and preventing ectopic neural stem/progenitor proliferation and medulloblastoma genesis. 


\section{TABLE OF CONTENTS}

CHAPTER 1. GENERAL INTRODUCTION ....................................................................1

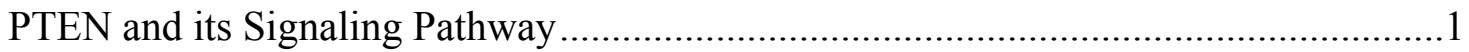

Identification of PTEN ................................................................................

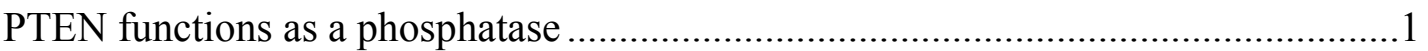

PTEN negatively regulates PI3K/AKT/mTOR pathway ...................................

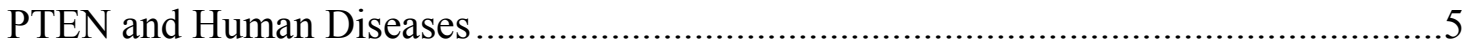

PTEN Knock-out Mouse Models outside of the Central Nervous System.....................5

PTEN in Neuropathology and Neural Development ............................................6

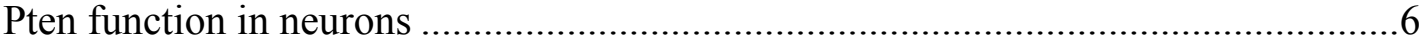

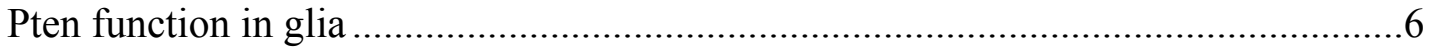

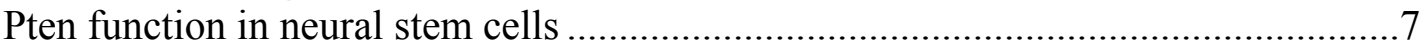

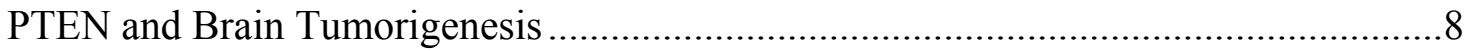

Postnatal brain development and neural stem cells in adulthood .............................8

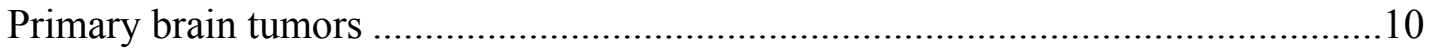

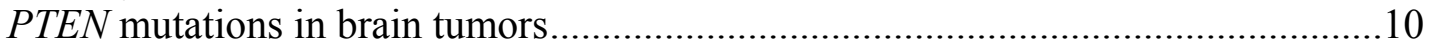

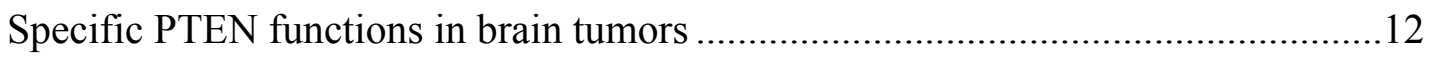

CHAPTER 2. PTEN DELETION IN THE SUBVENTRICULAR ZONE

CAUSES MTORC1-DEPENDENT ECTOPIC DIFFERENTIATION OF

NEUROBLASTS WITHOUT DISRUPTING DIRECTIONAL MIGRATION........15

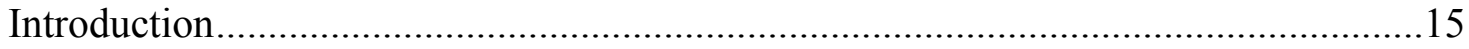

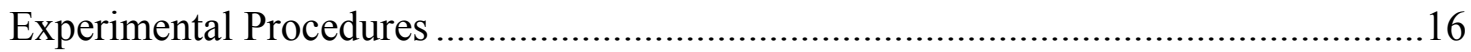

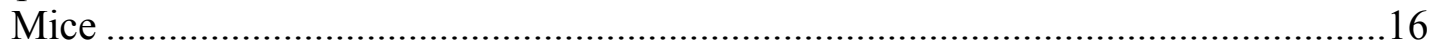

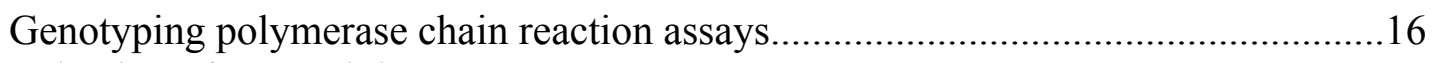

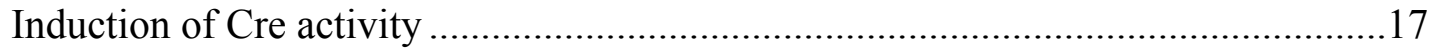

5-Bromo-2'-deoxyuridine injection and rapamycin treatment ............................... 17

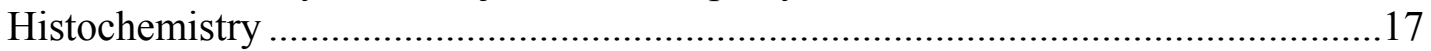

Immunohistochemistry and immunofluorescence .............................................18

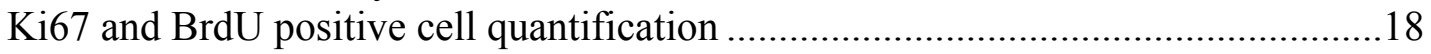

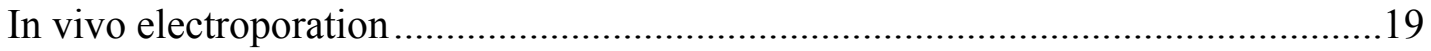

Brain slice preparation, time-lapse live imaging and migration analysis .................19

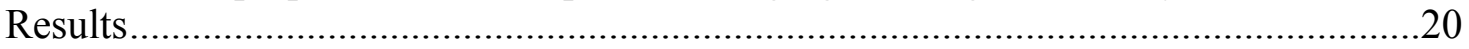

PI3K pathway activity in the wild-type SVZ-RMS-OB .........................................20

Postnatal deletion of Pten caused ectopic differentiated neurons in an expanded

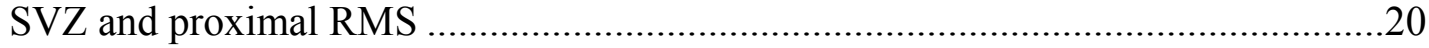

Pten loss did not induce substantial changes in proliferation or survival in the

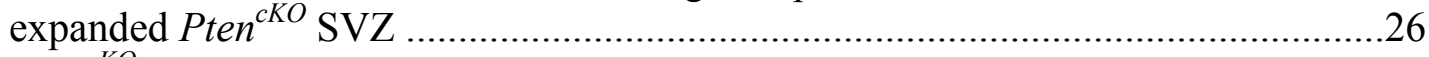

$\mathrm{Pten}^{c K O}$ neuroblasts terminated tangential migration prematurely in the SVZ

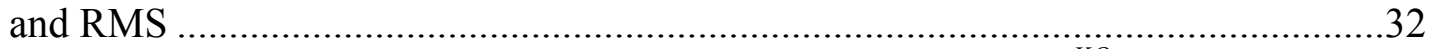

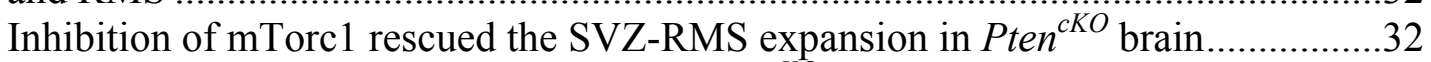

Ex vivo time-lapse live imaging showed $\operatorname{Pten}^{c K O}$ neuroblasts had normal

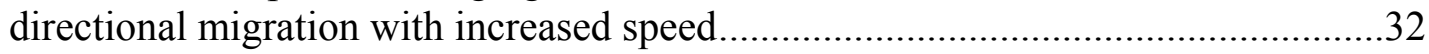

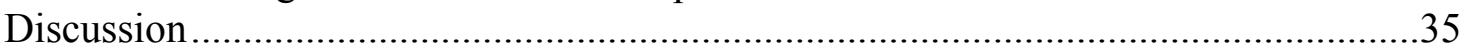


Pten function in neuronal migration and differentiation..............................................35

PI3K-Akt-mTorc1 pathway regulation in the SVZ-RMS-OB ...................................40

\section{CHAPTER 3. CO-DELETION OF PTEN AND TRP53 SYNERGIZES IN GENERATING MEDULLOBLASTOMA WITH A GROWTH PATTERN} CENTERING AROUND BLOOD VESSELS...........................................................42

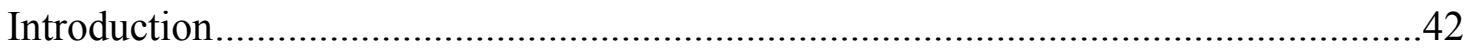

Experimental Procedures .............................................................................43

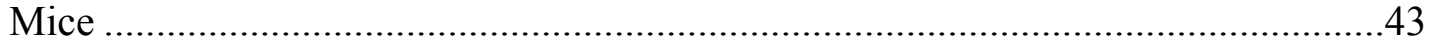

Genotyping polymerase chain reaction assays......................................................4

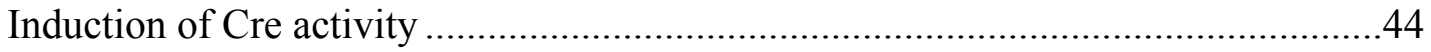

Immunohistochemistry and immunofluorescence …………..................................44

Array comparative genomic hybridization analysis...............................................4

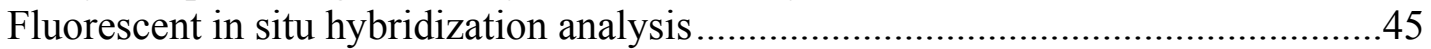

Quantitative real-time polymerase chain reaction assay ............................................45

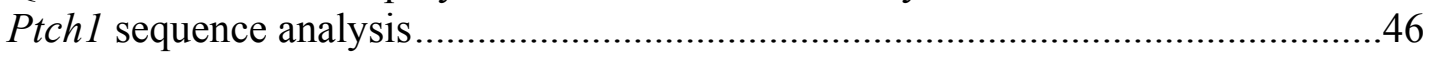

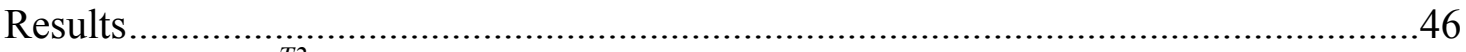

Nestin-CreER ${ }^{T 2}$ targets cerebellum at birth ..............................................................

Deletion of Pten at birth resulted in disorganization and hypertrophy of cerebellum which mimics human Lhermitte-Duclos disease ......................................48

Pten inactivation at birth revealed novel ectopic perivascular proliferative

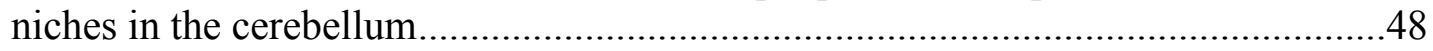

Co-deletion of Pten and Trp53 at birth resulted in medulloblastoma with unusual vascularization .......................................................................................

Pten;Trp53 double knock-out medulloblastoma showed a growth pattern centering around blood vessels ................................................................................51

Pten;Trp53 double knock-out medulloblastoma spontaneously lost

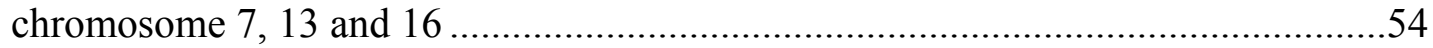

Spontaneous deletion and mutation of Ptchl occurred in Pten;Trp 53 double

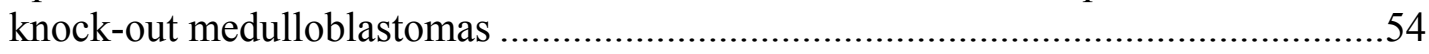

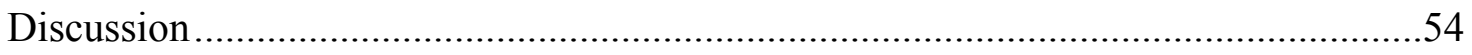

\section{CHAPTER 4. GENERAL DISCUSSION AND FUTURE DIRECTIONS .................59}

Pten and Postnatal Brain Development ......................................................................59

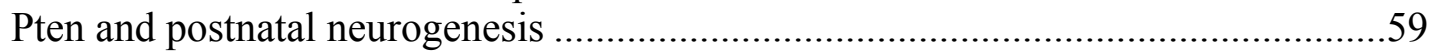

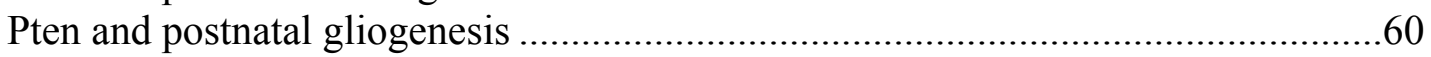

Remaining questions on Pten and development ……….......................................61

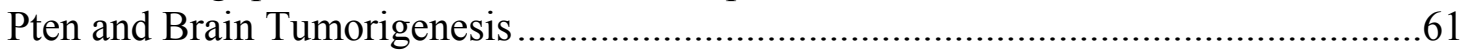

Cooperativity between Pten and other tumor suppressors .........................................61

Unified function of Pten in neurogenesis and medulloblastoma genesis?..................62

Why is the perivascular niche only seen in the cerebellum? .....................................62

Cells of origin of brain tumors...............................................................................63

Future directions for Pten;Trp 53 double knock-out medulloblastoma........................63

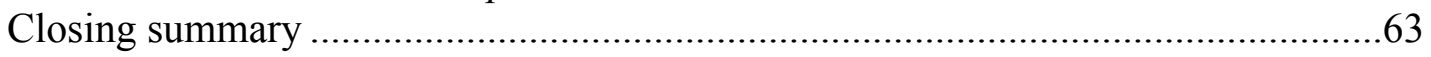

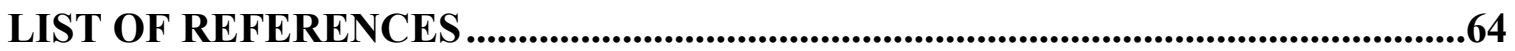


APPENDIX. SUPPLEMENTARY DATA...........................................................................83

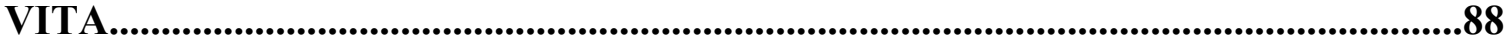




\section{LIST OF FIGURES}

Figure 1-1. Schematic PTEN secondary protein structure and biochemical function.......2

Figure 1-2. Schematic PI3K signaling pathway. ...................................................

Figure 1-3. Postnatal development of mouse brain. .................................................

Figure 1-4. Distributions of primary brain and CNS tumor by histology. ....................11

Figure 2-1. The PI3K-mTorc1 pathway was inactive in migrating SVZ neuroblasts,

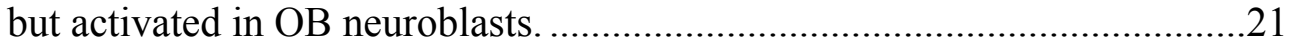

Figure 2-2. Nestin-CreER targeted neural stem/progenitor cells in the SVZ.................22

Figure 2-3. Postnatal Pten deletion caused ectopic differentiated neurons in an expanded SVZ.............................................................................24

Figure 2-4. Expansion and ectopic neuronal differentiation in the RMS.....................25

Figure 2-5. Ectopic cells in the expanded cKO SVZ expressed markers of mature neurons.

Figure 2-6. Reduced diameter of the terminal RMS and decreased granule cell

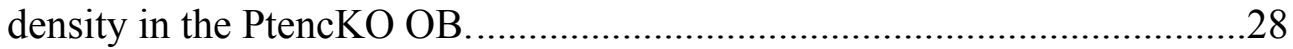

Figure 2-7. Pten $^{c K O}$ neuroblasts terminated tangential migration prematurely in the SVZ

Figure 2-8. mTorc1 inhibition did not affect proliferation or apoptosis in the SVZ......30

Figure 2-9. $\quad$ Pten $^{c K O} \mathrm{SVZ}$ neural progenitors were sensitive to irradiation and showed no change in apoptosis compared to control mice.

Figure 2-10. mTorc1 inhibition rescued the expansion of Pten $^{c K O}$ SVZ .........................33

Figure 2-11. EYFP+ cells in the Pten ${ }^{c K O}$ RMS and OB were Pten-null..........................36

Figure 2-12. Ex vivo time-lapse live imaging showed $\operatorname{Pten}^{c K O}$ neuroblasts had normal directional migration with faster speed.

Figure 2-13. The arrested EYFP+ cells in the Pten $^{c K O}$ RMS were in the neuronal lineage.

Figure 3-1. Nestin-CreER ${ }^{T 2}$ targets cerebellum at birth.

Figure 3-2. Deletion of Pten at birth resulted in disorganization and hypertrophy of cerebellum. 
Figure 3-3. Pten inactivation at birth reveals novel perivascular proliferative niches in the cerebellum.

Figure 3-4. Co-deletion of Pten and Trp53 at birth resulted in medulloblastoma with unusual vascularization.

Figure 3-5. Pten;Trp53 double knockout medulloblastoma showed a growth pattern centering around blood vessels.

Figure 3-6. Pten;Trp53 double knockout medulloblastoma spontaneously lost chromosome 7, 13 and 16 .

Figure 3-7. Spontaneous deletion and mutation of Ptch1 in Pten;Trp53 double knockout medulloblastoma.

Figure A-1. Increased number and size of astrocytes in the expanded Pte ${ }^{c K O}$ SVZ......83

Figure A-2. Deletion of Pten synergizes with Trp53 mutations to drive high-grade gliomas in adult brain arising from the SVZ.

Figure A-3. Nestin-CreER ${ }^{T 2} ;$ Pten $^{c K O} ;$ Tp $53^{c H E T}$ mice developed highly infiltrative gliomas throughout brain.

Figure A-4. Highly infiltrative gliomas developed in Nestin-CreER ${ }^{T 2}$ Pten $^{c K O}$; Tp $53^{\text {cHET }}$ mice. 


\section{LIST OF ABBREVIATIONS}

aCGH

$\mathrm{aCSF}$

bHLH

BAC

BrdU

BRRS

CB

CNS

CS

DAPI

DMSO

DPI

EGL

FISH

FRB

GABA

GAP

GBM

GCL

GTP

hPLAP

HBSS

IGL

IRES

LDD

LV

mTORC

ML

MMAC

OB

PBD

PBS

PCR

PDK1

PDZ

PEST

PFA

$\mathrm{PH}$

PHTS

PI3Ks

PIP2

PIP3 array comparative genomic hybridization

artificial cerebrospinal fluid

basic helix loop helix

bacterial artificial chromosome

5-Bromo-2'-deoxyuridine

Bannayan-Riley-Ruvalcaba syndrome

cerebellum

central nervous system

Cowden syndrome

4',6-diamidino-2-phenylindole

dimethyl sulfoxide

days post induction

external granule layer

fluorescent in situ hybridization

FKBP-rapamycin-binding

$\gamma$-Aminobutyric acid

GTPase-activating protein

glioblastoma multiforme

granule cell layer

guanosine triphosphate

human placental alkaline phosphatase

Hank's balanced salt solution

internal granule layer

internal ribosomal entry site

Lhermitte-Duclos disease

lateral ventricle

mammalian target of rapamycin complex

molecular layer

mutated in multiple advanced cancers

olfactory bulb

phosphatidylinositol-4,5-bisphosphate binding domain

phosphate buffered saline

polymerase chain reaction

phosphoinositide-dependent kinase 1

postsynaptic density protein-Drosophila disc large tumor suppressor-zonula occludens 1 protein

proline, glutamic acid, serine, threonine

paraformaldehyde

pleckstrin homology

PTEN hamartoma tumor sydrome

phosphatidylinosito-3-OH kinases

phosphatidylinositol-4,5-bisphosphate

phosphatidylinositol-3,4,5-trisphosphate 
PNET

PNS

PSL

PTEN

RMS

RTKs

RT-PCR

SGZ

SHH

SVZ

TEP1

TM

TUNEL

WHO primitive neuroectodermal tumours

peripheral nervous system

Proteus-like syndrome

phosphatase and tensin homolog deleted on chromosome 10

rostral migratory stream

receptor tyrosine kinases

reverse transcriptase polymerase chain reaction

subgranular zone

sonic hedgehog

subventricular zone

transforming growth factor $\beta$-regulated and epithelial cell enriched phosphatase 1

tamoxifen

terminal deoxynucleotidyl transferase-mediated nick end labeling World Health Organization 


\title{
CHAPTER 1. GENERAL INTRODUCTION
}

\author{
PTEN and its Signaling Pathway
}

\section{Identification of PTEN}

PTEN (Phosphatase and Tensin homolog deleted on chromosome 10) also known as MMAC (mutated in multiple advanced cancers) or TEPI (transforming growth factor $\beta$-regulated and epithelial cell enriched phosphatase 1) was originally identified in 1997 as a putative tumor suppressor gene on chromosome 10q23.3, a genomic region commonly deleted in several types of human cancers including endometrial, prostate, breast cancers and glioblastoma multiforme (GBM) (Li and Sun 1997; Li et al. 1997; Steck et al. 1997).

\section{PTEN functions as a phosphatase}

The structure of PTEN protein organizes into four functional domains: N-terminal phosphatidylinositol-4,5-bisphosphate (PIP2)-binding domain (PBD), a phosphatase domain, a $\mathrm{C} 2$ domain for membrane recruitment and a $\mathrm{C}$-terminus containing two PEST sequences for proteolytic degradation and a PDZ-binding motif for protein-protein interaction (Figure 1-1A), (Georgescu et al. 1999; Lee et al. 1999; Georgescu et al. 2000; Vazquez et al. 2001; Das et al. 2003). As indicated in its structure, PTEN shares sequence homology with the family of protein-tyrosine phosphatases and can dephosphorylate serine, threonine, and tyrosine residues on peptide substrates in vitro (Myers et al. 1997). However, no protein substrate has been convincingly identified in vivo so far and the best characterized function of PTEN is associated with its lipid phosphatase activity with which PTEN catalyzes dephosphorylation of phosphatidylinositol-3,4,5-trisphosphate (PIP3) at position 3 on the inositol ring to generate PIP2 (Maehama and Dixon 1998). This lipid phosphatase activity is direct antagonism to that of phosphatidylinositol-3-OH kinases (PI3Ks) which phosphorylates PIP2 to PIP3 and activate downstream pathway (Figure 1-1B).

\section{PTEN negatively regulates $\mathrm{PI3K} / \mathrm{AKT} / \mathrm{mTOR}$ pathway}

PI3Ks are an evolutionarily conserved family of lipid kinases that phosphorylate the 3'-hydroxyl group of phosphatidylinositol and phosphoinositides, the crucial regulators of a variety of cellular processes (Engelman et al. 2006). PIP2, one kind of substrates for PI3Ks, is phosphorylated in response to activation of receptor tyrosine kinases (RTKs) or G-protein coupled receptors on the plasma membrane creating PIP3 (Engelman et al. 2006). PIP3 acts as a second messenger recruiting the serine/threonine kinase AKT to the membrane via its pleckstrin homology $(\mathrm{PH})$ domain. AKT is phosphorylated at threonine 308 by another serine/threonine phosphoinositide-dependent 
A
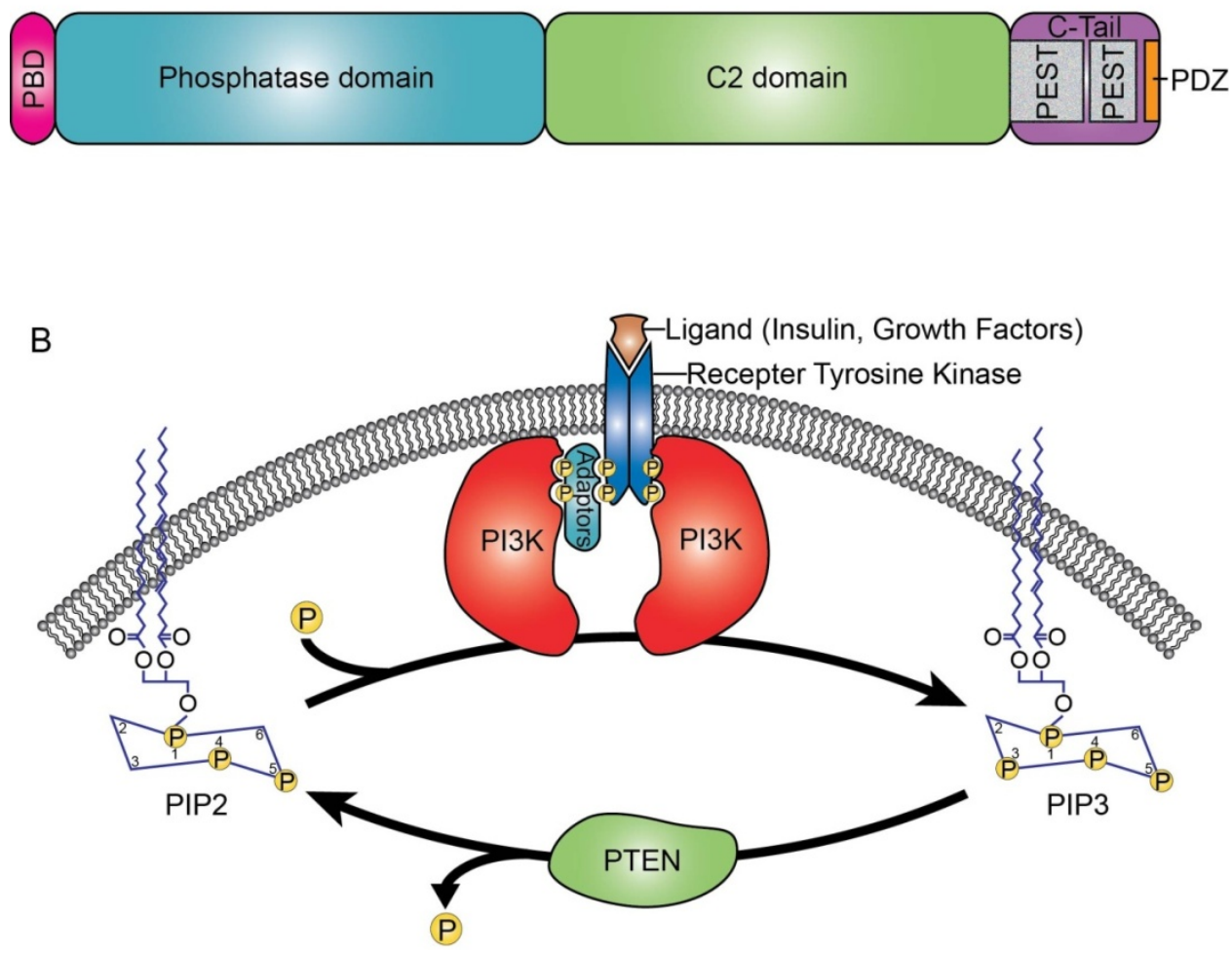

Figure 1-1. Schematic PTEN secondary protein structure and biochemical function.

(A) The structure of PTEN protein organizes into four functional domains: N-terminal PIP2-binding domain (PBD), a phosphatase domain, a C2 domain for membrane recruitment, and a $\mathrm{C}$-terminus containing two PEST sequences for proteolytic degradation and a PDZ-binding motif for protein-protein interaction. (B) PTEN functions as a phosphatase which dephosphorylates membrane-bound phospholipid PIP3 at position 3 on the inositol ring to generate PIP2. This dephosphorylation is a reverse reaction to the phosphorylation by PI3K downstream RTK activation. Modified with permission. Chalhoub, N. and Baker, S.J. 2009. PTEN and the PI3-kinase pathway in cancer. Annu Rev Pathol 4: 127-150. Courtesy of Dr. Suzanne Baker. 
kinase 1 (PDK1) recruited by binding to PIP3 as well (Alessi et al. 1997). To be fully activated, AKT needs also to be phosphorylated at serine 473 by the mammalian target of rapamycin complex 2 (mTORC2) (Sarbassov et al. 2005). By converting PIP3 back to PIP2, PTEN antagonizes PI3K activity, limits and ultimately terminates PI3K-AKT signaling in cells and this lipid phosphatase ability has been shown to be essential for PTEN tumor suppressor function (Sansal and Sellers 2004) (Figure 1-2).

Activated AKT is able to phosphorylate a diverse set of substrates such as GSK3 $\beta$, p27KIP1, MDM2, BAD, FOXOs, IKK, TSC2 and PRAS40 to regulate cell cycle progression, cell survival, cell growth and cell metabolism (Engelman et al. 2006; Shaw and Cantley 2006). Among the variety of pathways downstream of AKT, mTORC1 (one of two complexes nucleating mTOR, the other is mTORC2) is essential in regulating cell growth through coordinating anabolism including macromolecule synthesis and nutrient storage and catabolism like autophagy and utilization of energy stores in response to growth factors, nutrients, energy and oxygen level etc. environmental cues (Sengupta et al. 2010). mTORC1 is regulated by direct modification of its components or modulation of Rheb, a small GTPase that binds and activates mTORC1 when bound to GTP. AKT phosphorylates PRAS40 and prevents its binding and inhibition of mTORC1 (Sancak et al. 2007; Vander Haar et al. 2007). AKT could also activate mTORC1 by phosphorylating and suppressing TSC2 which, together with its partner TSC1, is a GTPase-activating protein (GAP) for Rheb (Sancak et al. 2007) (Figure 1-2).

mTORC1 promotes protein synthesis through two of its substrates, the S6 kinases (S6Ks) and 4E-BPs which enhance ribosome assembly and mRNA translation. mTORC1 inhibits autophagy by modulating Atg1/ULK activity in the presence of nutrients and growth factors. mTORC1 also controls numerous metabolic pathways including glycolysis, sterol and lipid biosynthesis as well as nucleotide metabolism (Sengupta et al. 2010). Upon activation by growth factors, mTORC1 limits the extent of upstream growth factor signaling by a "negative feedback loop". The best characterized mechanism of the "negative feedback loop" is through S6K1 phosphorylation of IRS1 which interferes with IRS1 binding to insulin receptor and promotes its degradation, thus preventing overactivation of the pathway (Harrington et al. 2004; Shah and Hunter 2006). Many mTORC1 functions were first discovered by use of the small-molecule rapamycin, an allosteric inhibitor of mTOR. Rapamycin forms a complex with protein FKBP12 which binds to FRB domain of mTOR and somehow only inhibits mTORC1 activity in acute treatment (Huang et al. 2003). For long-term or high dose treatment, rapamycin could also inhibit mTORC2 activity by interfering with its assembly (Sarbassov et al. 2006). Inhibition of mTORC1 by rapamycin releases the "negative feedback loop" and results in enhanced PI3K signaling to AKT (O'Reilly et al. 2006).

Deletion of PTEN will lead to unopposed and constitutive activation of the PI3KAKT-mTORC1 pathway. Increases in phosphorylation of AKT serine 473 and threonine 308, phospho-S6 serine 235/236 (substrate of S6K) and phospho-4EBP1 threonine 37/46 are often used as the readouts of activated PI3K-AKT-mTORC1 pathway. 


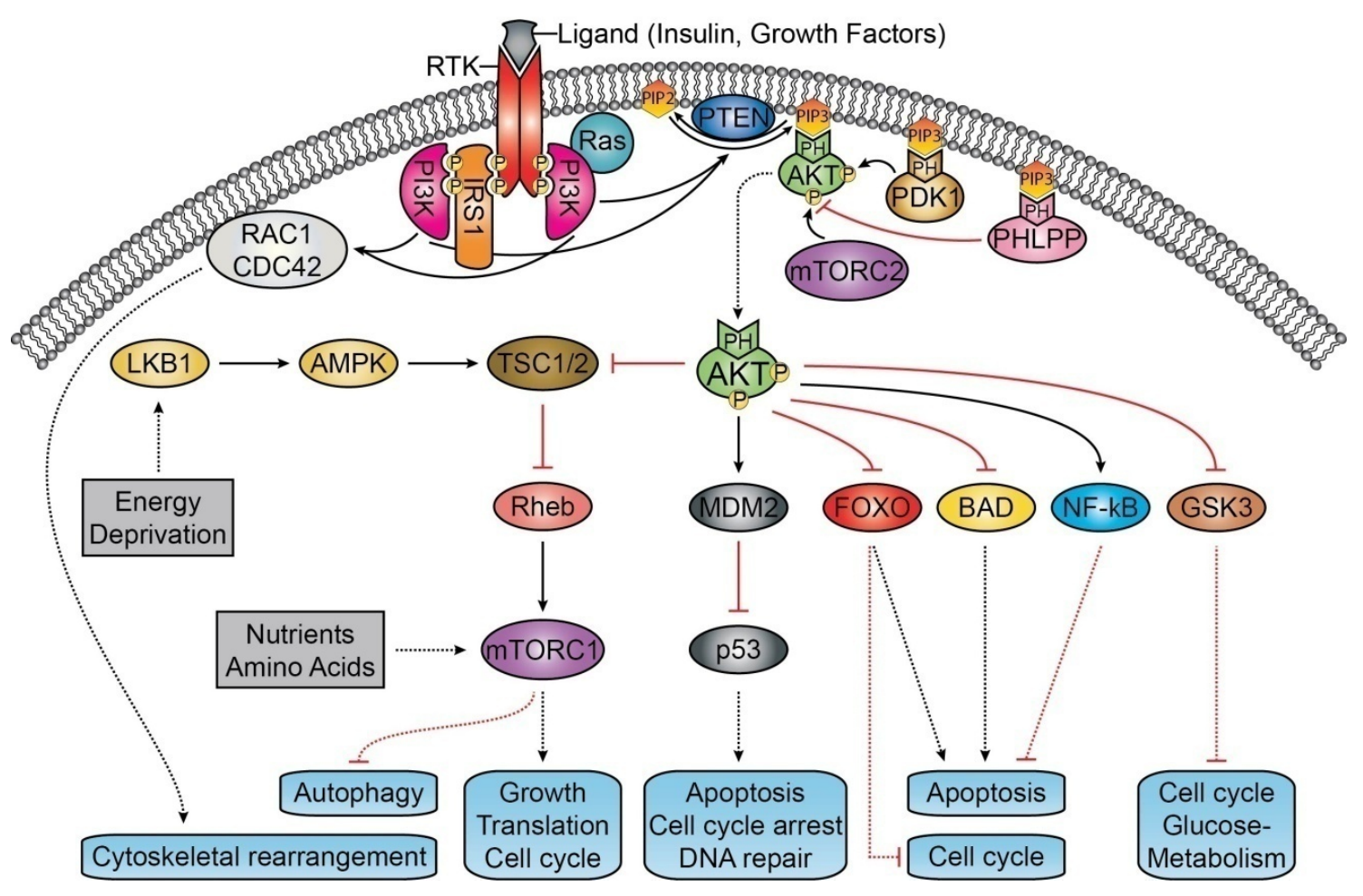

Figure 1-2. Schematic PI3K signaling pathway.

The RTK is activated when binding to its ligand and recruits PI3K which phosphorylates membrane-bound PIP2 to PIP3. PIP3 recruits AKT and PDK1 to the membrane through their PH domain. AKT is fully activated by phosphorylations by PDK1 and mTORC2. Activated AKT phosphorylates a diverse set of substrates such as TSC1/2, MDM2, FOXO, BAD, NF-kB and GSK3 to regulate processes including cell growth, cell-cycle apoptosis, cell metabolism and autophagy. By converting PIP3 back to PIP2 antagonizing PI3K activity, PTEN limits and terminates PI3K-AKT signaling. Reprinted with permission. Chalhoub, N. and Baker, S.J. 2009. PTEN and the PI3-kinase pathway in cancer. Annu Rev Pathol 4: 127-150. Courtesy of Dr. Suzanne Baker. 


\section{PTEN and Human Diseases}

Germline PTEN mutations have been found to occur in several inherited cancer and developmental syndromes including $80 \%$ of Cowden syndrome (CS), $60 \%$ of Bannayan-Riley-Ruvalcaba syndrome (BRRS), up to $20 \%$ of Proteus syndrome (PS), $50 \%$ of Proteus-like syndrome (PSL) (Eng 2003) and 83\% of Lhermitte-Duclos disease (LDD) (Zhou et al. 2003). These seemingly unrelated hereditary syndromes are now categorized under a unifying molecular-based diagnosis PTEN Hamartoma Tumor Syndrome (PHTS) since they share the clinical manifestation of multiple hamartomas and have overlapping mutational spectra of PTEN (Eng 2003). Several important clinical manifestations of PHTS patients include macrocephaly, multiple hamartomas and cancer predisposition in Cowden disease to breast, thyroid, endometrial, colorectal, and renal cancers (Riegert-Johnson et al. 2010).

Besides germline mutation, PTEN is also found to be frequently mutated in a wide variety of sporadic human cancers. The list of human cancers that harbor PTEN mutation has expanded from endometrial, prostate, breast cancers and GBM initially reported in 1997 to more than ten types (http://www.sanger.ac.uk/genetics/CGP/cosmic/), placing it as the second most frequently mutated gene in human cancer. In no doubt this list will keep expanding with the advancement of whole genome sequencing technology.

\section{PTEN Knock-out Mouse Models outside of the Central Nervous System}

Pten function in development and tumor suppression has been well characterized using traditional germline and conditional gene knock-out strategies. Homozygous deletion of Pten resulted in embryonic lethality by day 6.5-9.5 because of disrupted development of germ layers, while heterozygous mutant mice are predisposed to a variety of tumors including lymphomas, intestinal polyps, endometrial hyperplasia, prostate intraepithelial neoplasia and thyroid neoplasms (Di Cristofano et al. 1998; Suzuki et al. 1998; Podsypanina et al. 1999). This spectrum of neoplasias is similar to those in PHTS patients.

Cre-LoxP system has allowed people to bypass the embryonic lethality of $\mathrm{Pten}^{-/}$ mice and to study Pten function in a tissue-specific manner. Deletion of Pten in T cells resulted in hyperproliferation, auto-immunity and T-cell lymphoma (Suzuki et al. 2008). Pten deletion in skin lead to epidermal hyperplasia, hyperkaratosis, squamous papillomas, squamous cell carcinomas, sebaceous carcinomas and sweat gland adenocarcinomas (Suzuki et al. 2008). Hepatocyte-specific Pten knock-out caused massive hepatomegaly, steatohepatisis, liver adenomas and hepatocellular carcinomas (Suzuki et al. 2008). Deletion of Pten in urothelial cells resulted in urothelial hyperplasia and hypertrophy and transitional cell carcinomas (Suzuki et al. 2008). Most of mice with bronchioalveolar epithelium-specific inactivation of Pten died of hypoxia at birth and the rest developed spontaneous lung adenocarcinomas (Suzuki et al. 2008). Pten is also required for normal blood vessel remodeling and suppressing tumor angiogenesis (Suzuki et al. 2008). Pten has an essential role in restricting the activation of hematopoietic stem 
cells and preventing leukaemogenesis. Inactivation of Pten in the bone marrow resulted in a short-term expansion but long-term decline of hematopoietic stem cells and eventual leukemia formation (Yilmaz et al. 2006; Zhang et al. 2006). Oocyte-specific deletion of Pten causes premature activation of the primordial follicle pool and premature ovarian failure (Reddy et al. 2008). Conditional Pten knock-out mice have also been generated that develop teratomas, pancreatic cancers, cholangiocellular carcinomas and leiomyosarcomas (Suzuki et al. 2008).

\section{PTEN in Neuropathology and Neural Development}

\section{Pten function in neurons}

PTEN inactivation in the cerebellum causes LDD or dysplastic gangliocytoma of the cerebellum, a hamartomatous lesion in the cerebellum. LDD patients manifest with progressive macrocephaly, seizure, ataxia and mental retardation. The typical pathological feature of LDD is the diffusely hypertrophic and disorganized cerebellar folia with replacement of the Purkinje and granule cells by hypertrophic ganglionic neurons (Abel et al. 2005). The features of LDD were recapitulated by mouse models in which Pten was conditionally deleted in cerebellar granule cells during development. Pten deletion resulted in progressive increase in brain size, seizures, neuronal hypertrophy and migration defect demonstrating that Pten regulates neuronal size and migration (Backman et al. 2001; Kwon et al. 2001). PHTS patients also exhibit autistic behavior and mental retardation with decreased or delayed learning (Goffin et al. 2001; Eng 2003). Interestingly, a mouse model deleting Pten in differentiated neurons in the cerebral cortex and hippocampus showed similar autistic features to human patients including abnormal social interaction, anxiety-like behavior and decreased learning (Kwon et al. 2006). These neurological and behavioral manifestations could be presumably explained by the abnormalities in neuronal structure and function caused by Pten deficiency such as hypertrophic and ectopic dendrites, increased dendritic spine density, increased axonal synapses, severe myelination defects, weakened synaptic transmission and synaptic plasticity (Kwon et al. 2006; Fraser et al. 2008).

\section{Pten function in glia}

The axons of neurons are usually ensheathed by myelin which is formed by oligodendrocytes in the central nervous system (CNS) or Schwann cells in the peripheral nervous system (PNS). Abnormalities in myelination underlie a variety of human diseases such as multiple sclerosis, Guillain-Barré Syndrome, central pontine myelinosis, inherited demyelinating diseases like Leukodystrophy and Charcot Marie Tooth (Suter and Scherer 2003). One histopathological feature in LDD patients is the abnormal myelination in the cerebellar molecular layer (Abel et al. 2005) suggesting Pten might be involved in the regulation of myelination. Recent studies in mouse models just started to reveal Pten function in the oligodendrocytes and Schwann cells. Pten deletion in either 
developing or differentiated oligodendrocytes and Schwann cells resulted in dramatic hypermyelination (Fraser et al. 2008; Goebbels et al. 2010; Harrington et al. 2010). The hypermyelination was caused by oligodendrocyte hypertrophy and increased thickness of myelin sheath but not change in the number of oligodendrocyte in the CNS. In the PNS, however, Pten loss caused an increased number of Schwann cells and de novo myelination of normally non-myelinated nerve fibers as well as increased thickness of myelin sheath. Rapamycin treatment strongly impeded the volume increase of the white matter indicating that the AKT/mTOR pathway is one critical downstream effector in the differentiation of oligodendrocytes (Goebbels et al. 2010). There was also massive axon degeneration associated with progressive myelin sheath abnormalities. However, there was no detectable improvement in remyelination following white matter injury. These studies indicate that Pten is required to regulate myelin thickness and preserve axon integrity but dispensable during myelin repair (Harrington et al. 2010). Pten is also required for the Dlg1-mediated myelination brake whose failure causes Schwann cell hypermyelination with focally folded myelin, a hallmark of one type of Charcot-MarieTooth disease (Cotter et al. 2010). In addition, Pten also inhibits oligodendrocyte progenitor cell proliferation and promotes their differentiation (Paintlia et al. 2010).

Astrocytes are another large population of glial cells that provide structural support, regulate water balance and ion distribution and maintain the blood-brain barrier etc (Rowitch and Kriegstein 2010). Only very few studies have been conducted on Pten function in the development of astrocytes in their physiological environment. A mouse model inactivating Pten in both astrocytes and neurons during development showed increased cell size and proliferation in the astrocytes in contrast to the increase in cell size only in the neurons (Fraser et al. 2004). However, whether or not the hypertrophy and hyperproliferation in the astrocytes are cell-autonomous still needs further investigation because astrocytosis (an abnormal increase in the number of astrocytes) is a known phenomenon of astrocytes in response to environmental stresses such as neuron damage. Deletion of Pten in astrocytes in the adult brain resulted only in a slightly increased Gfap expression (Chow et al. 2011).

\section{Pten function in neural stem cells}

Inactivation of Pten by a Nestin promoter driven Cre in the neural stem/progenitor cells at midgestation demonstrated that mutant mice exhibit enlarged, histoarchitecturally disrupted brains, which resulted from increased cell proliferation, decreased cell death and enlarged cell size. Neurosphere cultures further showed increased proliferation and self-renewal capacity for Pten deficient neural stem/progenitor cells with no deficit in differentiation (Groszer et al. 2001; Groszer et al. 2006). These studies indicate Pten negatively controls proliferation and self-renewal of neural stem/progenitor cells during early brain development. However, the Nestin-Cre;Pten ${ }^{\text {loxP/loxP }}$ mice die soon after birth precluding investigation of Pten function in neural stem/progenitor cells in early postnatal days and adulthood. 


\section{PTEN and Brain Tumorigenesis}

\section{Postnatal brain development and neural stem cells in adulthood}

The bulk of brain structure changes relatively little after birth except the cerebellum, hippocampus and subventricular zone (SVZ) where neurogenesis and neuronal migration continue in postnatal days and adults. Although the establishment of the cerebellar territory and generation of Purkinje neurons and deep cerebellar nuclei happen during embryogenesis, most granule neurons are generated postnatally. Granule neuron progenitors migrate superficially from rhombic lip to form the external granule layer (EGL) on the surface of the cerebellum at embryogenesis. These cells continue proliferating and generate granule cells which descend into the cerebellum and form the internal granule layer (IGL) until postnatal day 21 in mouse and up to year 2 in human (Figure 1-3A). Active postnatal development makes cerebellum very susceptible to mutagenesis. It is the most common site of origin for pediatric solid tumors, accounting for $15.6 \%$ of all primary brain and CNS tumors in childhood (CBTRUS 2010).

Neural stem cells or neural precursor cells in adulthood are mainly restricted to the hippocampus and SVZ in the brain where neurogenesis occurs throughout life. In mouse, it has been shown that thousands of new neuronal precursors are born every day in the SVZ and migrate through the rostral migratory stream (RMS) to the olfactory bulb (OB) where they differentiate into interneurons and are integrated into local circuits. Migrating neuronal precursor cells or neuroblasts $(\mathrm{Dcx}+)$ are differentiated from Mash1+ transit amplifying cells which in turn are generated by Gfap+ neural stem cells in the SVZ (Alvarez-Buylla and Garcia-Verdugo 2002) (Figure 1-3B, C). SVZ has also been found to be conserved in human brain (Quinones-Hinojosa et al. 2006; Curtis et al. 2007). However whether or not the RMS is present in human brain is still controversial (Sanai et al. 2004; Curtis et al. 2007; Guerrero-Cazares et al. 2011). In hippocampus, new neurons are continously generated in the subgranular zone (SGZ) of dentate gyrus and migrate outward for a short distance to be incorporated into dentate gyrus. Neural stem/progenitor cells in the SVZ-RMS-OB and the SGZ are thought to play an important role in damage repair and the process of memory formation (Gage 2002; Ming and Song 2005;

Ghashghaei et al. 2007). They might also serve as cells of origin for brain tumors because of their great proliferative capacity.

Unlike the neurogenesis which occurs before gliogenesis during brain development and peaks at embryonic day 14 (E14), astrocytes and oligodendrocytes, two major glial cells in the brain are generated mainly postnatally peaking at P2 and P14, respectively (Sauvageot and Stiles 2002). Mature astrocytes and oligodendrocytes are able to proliferate in physiological condition or pathologically in response to injury. They are thought to be the other candidate cells of origin for primary brain tumors besides neural stem/progenitor cells. It is still largely unclear whether gliomas arise from neural stem/progenitor cells or from fully differentiated astrocytes or oligodendrocytes, the two most possible cell sources maintaining proliferation capacity in the brain for malignant 


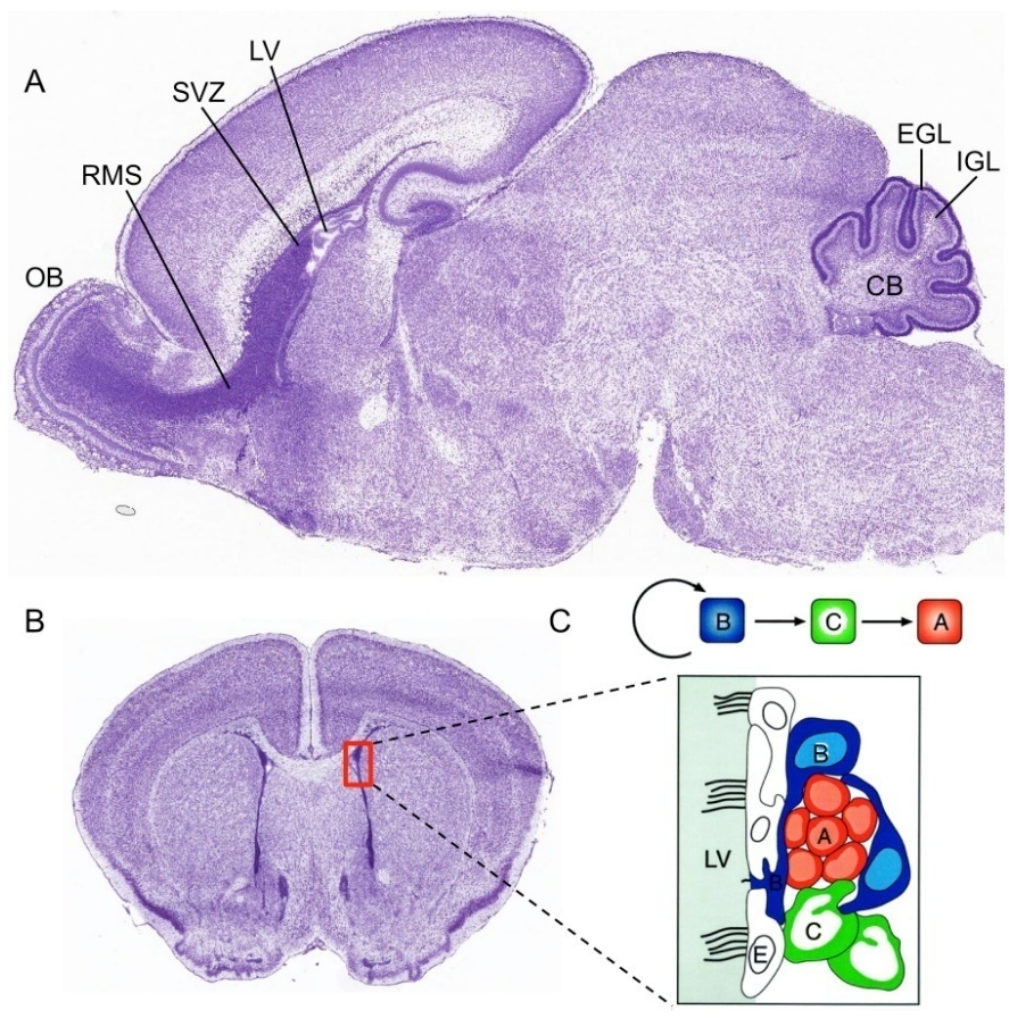

Figure 1-3. Postnatal development of mouse brain.

(A) Nissl staining of saggital mouse brain at age P4. Neurogenesis is still actively ongoing in postnatal brain in two places. First, new neuronal progenitors (or neuroblasts) arise from neural stem cells in the subventricular zone (SVZ) in the lateral walls of lateral ventricles (LV), migrate through the rostral migratory steam (RMS) to the olfactory bulb (OB) where they terminally differentiate and are integrated into local neural circuits. Second, granule cell progenitors actively proliferate in the external granule layer (EGL) of the cerebellum (CB), then migrate inward and differentiate forming the internal granule layer (IGL) during early postnatal days. (B) Nissl staining of coronal mouse brain of age P28 shows the SVZ. (C) Cell lineage in the SVZ. Neural stem cells (B cells) give rise to transit amplifying cells ( $\mathrm{C}$ cells) which in turn generate neuroblasts (A cells). $\mathrm{E}$ cells are ependymal cells lining the LV. Panel C is Modified with permission. AlvarezBuylla, A. and Garcia-Verdugo, J.M. 2002. Neurogenesis in adult subventricular zone. $J$ Neurosci 22(3): 629-634. 
transformation. The identification of cell origins of gliomas will not only improve our knowledge but also help develop therapeutic strategies of this disease.

\section{Primary brain tumors}

In the United States in the year 2010, there was an estimated 62,930 new cases of primary non-malignant and malignant brain and CNS tumors (CBTRUS 2010). Primary malignant brain and CNS tumors account for 1.4 percent of all cancers, 2.3 percent of all cancer deaths and 21.3 percent of pediatric cancers (ACS 2010). Meningioma, gliomas and pituitary tumor are the most common histology types of primary brain tumors in adults. Gliomas including astrocytoma, glioblastoma, oligodendroglioma, ependymoma and mixed gliomas etc. represent $32 \%$ of all primary brain tumors and $80 \%$ of malignant primary brain tumors. GBM (WHO grade IV) is the most malignant form and accounts for $53.8 \%$ of all gliomas (CBTRUS 2010) (Figure 1-4A). The median survival of GBM patients ranges from 9 to 12 month despite combined treatment of surgery, radiotherapy and chemotherapy. The survival rate for GBM has not been improved and no effective agents have emerged for over two decades (Furnari et al. 2007).

In children, brain tumors are the most common type of solid tumor and the incidence is about 4.7 per 100,000 person-years in the US. Pilocytic astrocytoma, malignant glioma and medulloblastoma are the most common individual histologies. Gliomas are the most frequent primary brain tumors in childhood accounting for about $50 \%$ of all tumors and $70 \%$ of malignant tumors. Embryonal tumors including medulloblastoma (WHO grade IV) are the second most common malignant form and account for $13.7 \%$ of all pediatric brain tumors among 0-14 years olds (CBTRUS 2010) (Figure 1-4B). While the 5-year survival rate of medulloblastoma patients has achieved $60 \%$ after treated with radio- and chemotherapy, the patients often suffer from side effects such as cognitive impairment and psychiatric disorders.

Overall, malignant glioma (WHO grade III and IV) and medulloblastoma, the most common primary brain tumors in adults and children, pose a big challenge to healthcare and await novel therapies.

\section{PTEN mutations in brain tumors}

One genetic abnormality that is frequently seen in adult and pediatric brain tumors is the loss of chromosome 10q where PTEN is located. Losses of chromosome $10 \mathrm{q}$ have been reported to occur in 60-80\% of GBM (Ohgaki and Kleihues 2007) and 25-30\% of medulloblastoma (McCabe et al. 2006; Rossi et al. 2006; Lo et al. 2007).

PTEN is reported to be mutated in $15-40 \%$ of GBM but is rare in low-grade gliomas (Ohgaki and Kleihues 2009) suggesting PTEN mutation is a late event in glioblastomagenesis. This is in contrast to some other mutations such as TP53 which occurs at the same frequencies in both low and high grade gliomas. Besides PTEN, 88\% 

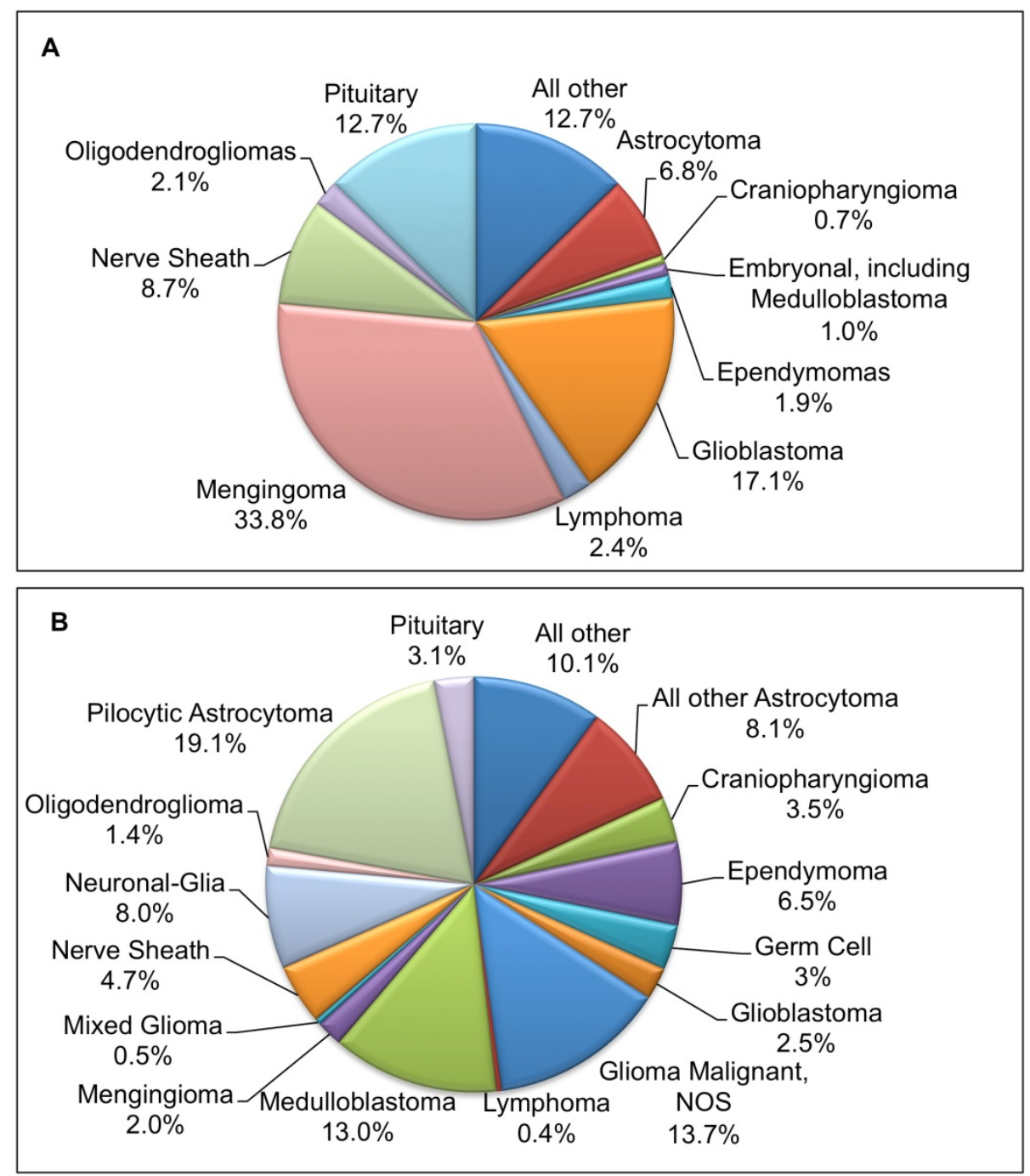

Figure 1-4. Distributions of primary brain and CNS tumor by histology. (A) Distribution of all primary brain and CNS tumors by histology. (B) Distribution of childhood primary brain and CNS tumors by histology (Ages 0-14). Source: CBTRUS. 2010. CBTRUS statistical report: primary brain and central nervous system tumors diagnosed in the United States in 2004-2006. Central Brain Tumor Registry of the United States, Hinsdale. 
of GBM samples harbored at least one genetic abnormality in the RTK/PI3K pathway including EGFR mutation and amplification (45\%), ERBB2 mutation (8\%), PDGFRA amplification (13\%), PIK3CA (15\%), NF1 mutation (18\%) and other less common genetic changes (TCGA 2008). RTK/PI3K pathway together with p53 (87\%) and RB $(78 \%)$ tumor suppressor pathways comprised the three core pathways that were altered in GBM (Parsons et al. 2008; TCGA 2008).

Numerous human genomic analyses and mouse modeling studies have underscored the pivotal role of the sonic hedgehog $(\mathrm{SHH})$ pathway in medulloblastoma pathogenesis. The binding of SHH to its receptor PTCH1 relieves its inhibition on SMO and allows the release of the transcription factor Gli from the inhibitory complex containing SUFU trans-activating downstream effector genes in the nucleus. SHH signaling plays an important role in driving proliferation of granule cell progenitors in the EGL of cerebellum. Inactivating mutations of $P T C H 1$ and $S U F U$ and activating mutations of $S M O$ have been found in $15-25 \%$ of sporadic medulloblastoma (Raffel et al. 1997; Reifenberger et al. 1998; Taylor et al. 2002; Parsons et al. 2011). The second signaling pathway that is frequently altered in the development of medulloblastoma is the Wnt pathway in which mutations in $A P C, A X I N$ or $C T N N B 1$ occur in $20 \%$ of the samples (Zurawel et al. 1998; Huang et al. 2000; Baeza et al. 2003; Parsons et al. 2011). Recent whole genome sequencing analysis also revealed new genetic alterations in this disease such as mutations in the histone-lysine N-methyltransferase MLL2 and MLL3 (20\%) and PTEN (3/88) as well as other genetic changes like TP53 (6/88), MYC (3/88) and OTX2 $(2 / 88)$ which have been implicated in medulloblastoma previously. Interestingly, three of the medulloblastomas with PTEN mutation also had either TP53 or PTCH1 mutation suggesting potential co-operational effect among them (Parsons et al. 2011).

\section{Specific PTEN functions in brain tumors}

Numerous studies in the past using glioma cell lines and xenograft models have revealed various aspects of PTEN tumor suppressor functions. For instance, re-expression of PTEN in the PTEN-deficient glioblastoma cell line could suppresses proliferation in vitro by arresting cells in the G1 phase of the cell cycle through a p27Kip1-dependent manner (Furnari et al. 1998; Gottschalk et al. 2001). Reintroduction of PTEN into human glioma cell lines could inhibit the in vitro invasiveness which is associated with reduced activity of matrix metalloproteinases including MMP2 and MMP9 (Koul et al. 2001; Kubiatowski et al. 2001). PTEN reconstitution also decreased the growth of orthotopic transplants of human glioma cell lines in nude mouse brain by reducing angiogenesis. This was due to PTEN function in induction of thrombospondin1 expression which inhibited angiogenesis and inhibition of HIF-1 $\alpha$ that induces angiogenesis (Wen et al. 2001; Pore et al. 2006). However most human glioma cell lines have been maintained and passaged in tissue culture for decades. They have adapted to animal serum that is different from the brain environment they originally arise from. These cell lines often do not show the classical histologic appearance of human gliomas any more (Lee et al. 2006a) and are not predictive for response for clinical trials (Voskoglou-Nomikos et al. 
2003). In addition, cell culture system is unable to model cancer invasion, angiogenesis, metastasis and the role of microenvironment in drug response.

Because of the limitations of cell line studies, genetically engineered mouse models with a close genetic resemblance to human disease have been utilized to better model human brain tumors. Several genetic mouse models of human glioma and medulloblastoma by knocking out Pten in combination with engineering of other diseaserelevant genes have been published in recent years that provide new insights into these diseases as well as Pten tumor suppressor function in the brain tumors. Specific deletion of Trp53 and Pten with Cre/loxP system in the mouse CNS generated spontaneous highgrade gliomas resembling human GBM. Inactivation of p53 and Pten promotes maintenance of mouse neural and glioma stem/progenitors in an undifferentiated state with high renewal potential by increasing Myc expression (Zheng et al. 2008). Haploinsufficiency of Pten could accelerate development of grade III astrocytomas generated by concomitant deletion of Trp53 and Nfl and loss of Pten heterozygosity promoted progression into GBM (Kwon et al. 2008). Further study demonstrated specific inactivation of Trp53, Nf1 and Pten in neural stem/progenitor cells in the SVZ was both necessary and sufficient to induce astrocytoma formation suggesting neural stem/progenitor as cells of origin for human GBM (Alcantara Llaguno et al. 2009). Another study provides similar evidence by showing that brain tumors arose from deleting Rb;Trp53, Rb;Trp53;Pten or Pten;Trp53 in adult SVZ neural stem cells but not in mature astrocytes. This study also demonstrates that genetic mutations might specify brain tumor phenotypes since inactivation of Pten;Trp53 gave rise to gliomas whereas deletion of $R b$; Trp 53 or $R b$;Trp53;Pten generated primitive neuroectodermal tumours (PNET) (Jacques et al. 2010). However, in another study all these combinations of tumor suppressor inactivation give rise to high-grade gliomas but not PNET (Chow et al. 2011). This discrepancy might be explained by the different strategies of gene inactivation between these two studies. The high-grade gliomas generated by Chow et al. (2011) mimic the expression profiling of human disease and arise from within and outside of the proliferative niches which demonstrates the relevance of the genetically engineered mouse models to human gliomas and also suggest that cells other than neural stem cells could serve as the origin of human gliomas.

In a mouse medulloblastoma model driven by Shh overexpression, Pten loss is shown to confer radiation resistance to cancer stem cells in the perivascular niche that is responsible for the tumor recurrence after irradiation (Hambardzumyan et al. 2008). Pten loss could also promote tumorigenesis in a mouse model of medulloblastoma driven by constitutive activation of SmoA1 and is associated with poor prognosis with clinical patients (Castellino et al. 2010). Interestingly, Pten is spontaneously lost in combination with biallelic inactivation of Ptch1 in the mouse medulloblastoma initiated with disrupted homologous recombination indicating a cooperation effect of PI3K and Shh pathway in medulloblastoma pathogenesis (Frappart et al. 2009).

Although past work has revealed various Pten functions in brain development and tumorigenesis, many questions still remain. How does loss of Pten cause the ectopic positioning of neurons? Is Pten involved in regulating directional migration as it is in 
Dictyostelium? How does the migration of Pten-null neuronal progenitors change compared to their wild-type counterparts? How will inactivation of Pten affect the neural stem/progenitor cells postnatally? Is loss of Pten in the neural stem/progenitors sufficient for brain tumorigenesis? What are other cells of origins for brain tumors besides neural stem cells in the SVZ? In the present study, we deleted Pten as well as other tumor suppressor genes in neural stem/progenitors using Nestin-CreER ${ }^{T 2}$ transgenic mice to address these questions. 


\title{
CHAPTER 2. PTEN DELETION IN THE SUBVENTRICULAR ZONE CAUSES MTORC1-DEPENDENT ECTOPIC DIFFERENTIATION OF NEUROBLASTS WITHOUT DISRUPTING DIRECTIONAL MIGRATION
}

\begin{abstract}
Introduction
In rodent brain, thousands of new neuronal precursors arise every day in the SVZ, the postnatal neural stem cell niche in the lateral walls of the lateral ventricles. Neuroblasts migrate from the SVZ through the rostral migratory stream (RMS) to the olfactory bulb (OB) where they differentiate into interneurons and are integrated into local circuits. Multiple cell types reside in this niche including Gfap-expressing neural stem cells which generate Mash1-expressing transit amplifying cells. Transit amplifying cells further differentiate into migrating neuronal precursor cells or neuroblasts that express the microtubule associated protein, Dcx (Alvarez-Buylla and Garcia-Verdugo 2002). In human brain, the SVZ niche harboring neural stem cells is conserved (Quinones-Hinojosa et al. 2006; Curtis et al. 2007), however the presence and cellular composition of the RMS remains somewhat controversial (Sanai et al. 2004; Curtis et al. 2007; Guerrero-Cazares et al. 2011). Although work conducted during recent years has unraveled many signals regulating the tangential migration from the SVZ to the OB, the extracellular cues and signaling pathways instructing when and where these tangentially migrating interneurons stop are largely unknown.

PI3Ks are an evolutionarily conserved family of lipid kinases that phosphorylate the 3'-hydroxyl group of phosphatidylinositol and phosphoinositides, essential regulators of diverse cellular processes including proliferation, survival, apoptosis, autophagy, growth, metabolism and cell migration (Engelman et al. 2006). Downstream of PI3K, Akt-mTorc1 signaling is a crucial branch of the pathway controlling protein synthesis, cell growth, autophagy and metabolism. Deregulation of the PI3K-Akt-mTorc1 pathway is associated with a variety of human genetic diseases, for instances, inherited mutations in TSC1 or TSC2 cause tuberous sclerosis; NF 1 mutation results in neurofibromatosis type 1; and PTEN mutations underlie CS, BRRS, LDD and Proteus syndrome (Inoki et al. 2005; Rosner et al. 2008). Patients with these inheritable diseases frequently manifest neurological abnormalities indicating that proper PI3K-Akt-mTorc1 pathway activity is important in normal neural development and/or function. Conditional mouse models knocking out the individual component genes in the brain recapitulate human diseases and further underscore the importance of PI3K-Akt-mTorc1 pathway regulation of neural development. Mice with neuron-specific ablation of Nfl have relatively normal neuronal development but display a reduced cerebral cortex and extensive reactive astrogliosis (Zhu et al. 2001). Deletion of Nf1 in astrocytes resulted in glial hyperplasia and optic nerve glioma (Bajenaru et al. 2003; Zhu et al. 2005) which is a hallmark of neurofibromatosis type1 patients. Mice deleted of Tsc1 or Tsc2 in neural progenitor or neurons demonstrated disrupted cortical and hippocampal lamination, ectopic dysplastic neurons as well as abnormal myelination and astrocytosis which recapitulate the neuropathology in tuberous sclerosis patients (Meikle et al. 2007; Way et al. 2009).
\end{abstract}


Pten, an important tumor suppressor gene, is a major negative regulator of the PI3K pathway. It functions as a lipid phosphatase antagonizing the activity of PI3Ks by catalyzing dephosphorylatation of PIP3 to PIP2. Pten plays a number of roles in brain development including negatively regulating self-renewal and proliferation of neural stem cells (Groszer et al. 2001) and controlling neuronal size (Backman et al. 2001; Kwon et al. 2001). A pronounced phenotype in the brain of Pten knock-out mice is the disrupted lamination and ectopic differentiation of neurons, which had been attributed to failed neuronal migration (Backman et al. 2001; Groszer et al. 2001; Kwon et al. 2001; Marino et al. 2002; Yue et al. 2005). However, the underlying mechanism of how Pten is involved in neuronal migration is currently unknown.

In the present study, we demonstrate that Pten inhibits downstream activation of mTorc1 in normal migrating neuroblasts in the RMS. Deletion of Pten in the SVZ and RMS caused ectopic differentiation of neuroblasts without disrupting the directional migration in the RMS.

\section{Experimental Procedures}

\section{Mice}

Nestin-CreER ${ }^{T 2}$ mice (Cicero et al. 2009) were generated using a transgene containing the Nestin promoter and second intron (Zimmerman et al. 1994) to direct expression of $\mathrm{CreER}^{\mathrm{T} 2}$ (Metzger et al. 1995; Feil et al. 1996; Feil et al. 1997), an internal ribosomal entry site (IRES), and human placental alkaline phosphatase (hPLAP) specifically in progenitor cells in the central nervous system. To map tamoxifen induction of Cre activity, Nestin-CreER ${ }^{T 2}$ transgenic mice were bred with $R 26 R-L a c Z$ (Soriano 1999) or R26R-EYFP (Srinivas et al. 2001) mice to generate Nestin-CreER ${ }^{T 2} ; R 26 R$-LacZ or Nestin-CreER ${ }^{T 2} ; R 26 R-E Y F P$ mice. Cre reporter activity was detected by X-gal staining or EYFP IF staining on frozen tissue sections. Pten conditional knock-out mice (Nestin-CreER $^{T 2}$; Pten ${ }^{\text {loxP/loxP }}$, or R26R-EYFP;Pten ${ }^{\text {loxP/loxP }}$ for Cre electroporation experiments) were generated by intercrossing Nestin-CreER ${ }^{T 2}$ or $R 26 R-E Y F P$ transgenic mice to Pten ${ }^{\operatorname{lox} P}$ mice (Suzuki et al. 2001), a gift from Tak Mak (University of Toronto, Toronto). For all analyses including Nestin-CreER ${ }^{T 2}$, the transgene was hemizygous to avoid possible variation from transgene dosage. Littermate Pten ${ }^{\text {loxP/loxP }}$ mice without the Nestin-CreER ${ }^{T 2}$ transgene were used as control and processed the same way as Pten ${ }^{\text {cKO }}$ mice. All procedures were reviewed and approved by the Animal Care and Use Committee at St. Jude Children's Research Hospital.

\section{Genotyping polymerase chain reaction assays}

Polymerase chain reaction (PCR) was used to genotype mice. Primers used are: Cre, forward primer: 5'AGCGATCGCTGCCAGGAT; reverse primer: 5'ACCAGCGTTTTCGTTCTGCC; expected product: 157 base pairs. Pten, forward 
primer: 5'TTATCTGGATCAACTTTGGGCC; reverse primer:

5'TCCCACCAATGAACAAACAGT; expected products: 146 (wild-type) and 244

(loxP) base pairs.

\section{Induction of Cre activity}

Tamoxifen (TM) (Sigma) was dissolved at $20 \mathrm{mg} / \mathrm{ml}$ or $5 \mathrm{mg} / \mathrm{ml}$ at $37^{\circ} \mathrm{C}$ in corn oil (Sigma) for Cre induction in adult mice or pups respectively. Dissolved TM was sterilized by filtering and stored at $4^{\circ} \mathrm{C}$ in the dark for up to 10 days. Pups were injected with $3 \mathrm{mg} / 40 \mathrm{~g}$ TM introperitoneally (i.p.) daily from P0-1 or P11-12 as indicated. Adult mice were injected with $9 \mathrm{mg} / 40 \mathrm{~g}$ TM i.p. daily from P28-30. An equivalent volume of sterile filtered corn oil alone was used for vehicle injections. Multiple injections in the same mouse were separated by 24 hours.

\section{5-Bromo-2'-deoxyuridine injection and rapamycin treatment}

5-Bromo-2'-deoxyuridine (BrdU) (Sigma) was dissolved at $5 \mathrm{mg} / \mathrm{ml}$ in sterile phosphate buffered saline (PBS) and stored at $-20^{\circ} \mathrm{C}$. BrdU was injected i.p. at a dose of $50 \mu \mathrm{g} / \mathrm{g}$ body weight every 2 hours for 5 injections for short-term BrdU labeling or every 12 hours for 6 times from P4-P6 for BrdU birthdating experiment.

Rapamycin (LC Laboratories) was dissolved at $20 \mathrm{mg} / \mathrm{ml}$ in sterile dimethyl sulfoxide (DMSO), stored at $-20^{\circ} \mathrm{C}$ and diluted in $5.2 \%$ Tween 80 as working solution before use. Rapamycin was injected i.p. at $1.5 \mu \mathrm{g} / \mathrm{g}$ body weight daily from P8-P31 of age. An equivalent volume of sterile DMSO+Tween 80 alone was used for vehicle injections. When TM was given on the same day, the two drugs were separated by 6 hours.

\section{Histochemistry}

Mice were anesthetized and perfused transcardially with PBS followed by $2 \%$ paraformaldehyde (PFA) in PBS. Following dissection, tissues were post-fixed overnight in $2 \% \mathrm{PFA}$ in PBS at $4{ }^{\circ} \mathrm{C}$, and then equilibrated in $25 \%$ sucrose in PBS for an additional 24 hours at $4^{\circ} \mathrm{C}$. Tissues were embedded in TBS embedding media (Triangle Biomedical Sciences) on dry ice and cryosectioned at thickness of $12 \mu \mathrm{m}$. Tissue slides were equilibrated at room temperature for 20 minutes then washed three times in PBS prior to staining.

For detection of $\beta$-galactosidase activity, slides were washed in Rinse A $(100 \mathrm{mM}$ $\mathrm{NaPO}_{4}, \mathrm{pH} 7.3 ; 2 \mathrm{mM} \mathrm{MgCl} 2 ; 5 \mathrm{mM}$ EGTA) for 30 min followed by Rinse B (100 mM $\mathrm{NaPO}_{4} \mathrm{pH} 7.3 ; 2 \mathrm{mM} \mathrm{MgCl} 2 ; 0.01 \%$ sodium deoxycholate; $\left.0.02 \% \mathrm{NP}-40\right)$ for $5 \mathrm{~min}$. Slides were then stained at $37^{\circ} \mathrm{C}$ for overnight in Developing Buffer $(1 \mathrm{mg} / \mathrm{ml} \mathrm{X-gal;} 100$ $\mathrm{mM} \mathrm{NaPO}_{4} ; \mathrm{pH} 7.3 ; 2 \mathrm{mM} \mathrm{MgCl} ; 0.01 \%$ sodium deoxycholate; $0.02 \% \mathrm{NP}-40 ; 5 \mathrm{mM}$ 
$\left.\mathrm{K}_{3} \mathrm{Fe}(\mathrm{CN})_{6} ; 5 \mathrm{mM} \mathrm{K}{ }_{4} \mathrm{Fe}(\mathrm{CN})_{6}\right)$. After washing in PBS, slides were counterstained with Nuclear Fast Red (Vector Labs).

\section{Immunohistochemistry and immunofluorescence}

Cryosections were prepared as described above. For paraffin sections, mice were processed the same way as above except the PFA concentration was 4\%PFA. Following perfusion, tissues were post-fixed for an additional 24 hours in 4\% PFA, embedded in paraffin, and cut into $5 \mu \mathrm{m}$ sections. Primary antibodies used for immunostaining were against GFP (1:1000, Invitrogen A6455; 1:2000, Abcam \#13970), Pten (1:100 for immunohistochemistry (IHC), 1:500 for immunofluorescence (IF) with Tyramide amplification, Cell Signaling \#9559), p-Akt S473 (1:50, Cell Signaling \#9271), p-S6 S235/236 (1:500, Cell Signaling \#2211), Dcx (1:4000 for IHC, 1:500 for IF, Chemicon AB5910), Mash1 (1:100, BD \#556604), NeuN (1:500, Chemicon), Map2 (1:5000, Sternberger SMI52), Calretinin (1:2000, Chemicon AB149), Gfap (1:200, Sigma G3893), Ki67 (1:5000, Novocastra NCL-Ki67p), BrdU (1:1000, AbDSerotec OBT0030CX) and active Caspase 3 (1:1000, BD \#559565). For IHC, microwave antigen retrieval was performed for all antibodies and biotinylated secondary antibodies were used in conjunction with horseradish peroxidase-conjugated streptavidin (Elite ABC, Vector Laboratories). Tyramide Signal Amplification (Perkin Elmer) was used to improve Pten IF and p-Akt S473 signal. Color development was conducted with substrates NovaRed, DAB or VIP (Vector Laboratories) and counterstaining with hematoxylin or methyl green (Vector Laboratories), respectively. For IF, Alexa Fluor 488, 647 (Invitrogen) and cyanine 3, 5 (Jackson Immunoresearch) conjugated secondary antibodies were employed along with Vectashield mounting media containing 4',6diamidino-2-phenylindole (DAPI) (Vector Laboratories). Terminal deoxynucleotidyl transferase-mediated nick end labeling (TUNEL) staining was conducted with the ApopTag peroxidase in situ apoptosis detection kit (Chemicon S7100) according to the manufacturer's protocol.

\section{Ki67 and BrdU positive cell quantification}

Ki67 or BrdU immunostaining from anatomically matched sections were chosen for quantification. For proliferation analyses, the Bioquant system (R\&M Biometrics) was used to count all the Ki67+ cells in the SVZ. For the BrdU birthdating experiment, BrdU+ cell number and granule cell density in a field of 20x objective in the same areas of the OB granule cell layer (GCL) or total number of BrdU+ cell in the SVZ were counted with ImageJ v1.44 cell counter. Four controls and four Pten ${ }^{c K O}$ brains were analyzed for each group and positive cells were counted from three sections from each brain for each staining. Student's t-test was used for statistical significance analysis. 


\section{In vivo electroporation}

In vivo electroporation was performed using a similar approach to previous reports (Boutin et al. 2008; Chesler et al. 2008). 2 ul of endotoxin-free $p C A G$-Cre expression plasmid $(0.65 \mu \mathrm{g} / \mu \mathrm{l}$, in PBS containing $1 \%$ fast green $)$ was injected into the lateral ventricle of $\mathrm{P} 2$ pups with a $30 \mathrm{G}$ needle Hamilton syringe using the midpoint between Bregma and Lambda and $1 \mathrm{~mm}$ lateral to the midline suture as landmarks for injection. Fast green dye allowed visual confirmation of correct injection into lateral ventricles. Only successfully injected animals were subjected to five square-wave electrical pulses (50 ms separated by $950 \mathrm{~ms}$ intervals, 150v). Electroporated pups recovered on a $37^{\circ} \mathrm{C}$ heating pad before returning to the mother.

\section{Brain slice preparation, time-lapse live imaging and migration analysis}

Mouse brains were quickly removed and placed in sterile filtered cold $\left(4^{\circ} \mathrm{C}\right)$ dissecting Complete Hank's Balanced Salt Solution (Complete HBSS) containing 1x HBSS (GIBCO), $2.5 \mathrm{mM}$ Hepes (pH 7.4, GIBCO), $30 \mathrm{mM}$ D-glucose, $1 \mathrm{mM} \mathrm{CaCl}_{2}, 1$ $\mathrm{mM} \mathrm{MgSO}_{4}$, and $4 \mathrm{mM} \mathrm{NaCO}_{3}$ (Polleux and Ghosh 2002). After dissection, brains were embedded in 3\% low-melting point agarose (Promega) and cut into $300 \mu \mathrm{m}$ thick sections saggitally with a vibratome. Slices containing the RMS were identified by EYFP expression, equilibrated for 2 hours in artificial cerebrospinal fluid (aCSF) containing 125 $\mathrm{mM} \mathrm{NaCl}, 2.5 \mathrm{mM} \mathrm{KCl}, 2 \mathrm{mM} \mathrm{CaCl}, 2 \mathrm{mM} \mathrm{MgCl}_{2}, 1.25 \mathrm{mM} \mathrm{NaH}_{2} \mathrm{PO}_{4}, 26 \mathrm{mM}$ $\mathrm{NaHCO}_{3}$, and $20 \mathrm{mM}$ D-glucose (285-295 mOsm) with $95 \% \mathrm{O}_{2} / 5 \% \mathrm{CO}_{2}$ at room temperature and then transferred into the submerged recording chamber and superfused (1-2 $\mathrm{ml} / \mathrm{min})$ with warm $\left(36-37^{\circ} \mathrm{C}\right)$ aCSF. Time-lapse live imaging was acquired by twophoton laser-scanning microscopy equipped with an Ultima imaging system (Prairie Technologies), a Ti:sapphire Chameleon Ultra femtosecond-pulsed laser (940 nm) (Coherent), and a 20x 0.95 numerical aperture water-immersion infrared objective (Olympus). Z stacks (512 x 512 pixels, $0.079 \mu \mathrm{m} /$ pixel, $60-100$ sections per stack, $1 \mu \mathrm{m} / \mathrm{section})$ were collected at the elbow of the RMS every 5 minutes for 3-6 hours.

Average projections of 20 consecutive sections in the middle of z-stacks of timelapse live imaging were analyzed using SlideBook v5.0 (Intelligent Imaging Innovations). Cells that exhibited bipolar morphology and moved at least two cell body lengths were counted as "bipolar migrating cells". All other cells were considered "stationary or non-polar cells". For each movie, all the EYFP+ cells were manually tracked but only those that could be observed more than 5 consecutive frames were included in the analysis. The average speeds (total displacement / time) or endpoint speeds (the distance between the first and last timepoints of the path / time) were binned in $0.005 \mu \mathrm{m} /$ second interval to obtain a distribution. $\chi^{2}$ analysis or Student's t-test was used to assess if the distributions or means, respectively, of average speeds (or endpoint speeds) were significantly different. 


\section{Results}

\section{PI3K pathway activity in the wild-type SVZ-RMS-OB}

To understand how PI3K signaling is regulated in the SVZ-RMS-OB, we examined expression of several key components of the pathway. Levels of Pten expression were heterogeneous in the SVZ and all of the cells expressing Pten were Dcx+ neuroblasts (Figure 2-1A). In the SVZ and proximal RMS, the majority of cells expressing $\mathrm{p}-\mathrm{S} 6$, a downstream indicator of PI3K/mTorc1 pathway activity, were Mash1+ transit amplifying cells, and not Dcx+ migrating neuroblasts (Figure 2-1B and data not shown). In contrast, in the terminal RMS at the OB core, a substantial population of Dcx+ type A cells expressed p-S6 (Figure 2-1C). This indicates that the PI3K pathway was not strongly active in migrating neuroblasts until these cells reached their destination in the OB.

\section{Postnatal deletion of Pten caused ectopic differentiated neurons in an expanded SVZ and proximal RMS}

To evaluate the function of the PI3K pathway regulator Pten in postnatal neural stem/progenitors, we generated Nestin-CreER ${ }^{T 2}$ mice with inducible Cre activity in the SVZ (Figure 2-2), and bred them to Pten ${ }^{\operatorname{lox} P / \operatorname{lox} P}$ (Suzuki et al. 2001) to generate inducible Pten conditional knockout mice (hereafter $\operatorname{Pten}^{c K O}$ ). Induction of Pten deletion in adult SVZ by TM injection in Pten ${ }^{c K O}$ mice at P30-P32 did not cause obvious neurological abnormalities throughout a normal lifespan except for occasional seizures in some aged animals. Analysis of all Pten ${ }^{c K O}$ brains $(n=24)$ showed dramatic expansion of the SVZ (Figure 2-3A) and proximal RMS (Figure 2-4). The expansion was most pronounced at the dorsal junction of the SVZ abutting the corpus callosum and the ventral tip where it formed a bulb-like structure. The expansion started to appear as early as 20 days post induction (DPI) and was similar at 60, 150, 300, and 540 DPI without progressive enlargement at later time points. IHC analysis showed the expanded SVZ was uniformly negative for Pten staining with marked elevation of $\mathrm{p}$-Akt indicating overall activation of the PI3K pathway in the entire region (Figure 2-3B, C).

When conditional Pten deletion was induced in newborn Pten $^{c K O}$ mice by TM injection at $\mathrm{P} 0$ and $\mathrm{P} 1$, similar SVZ and RMS expansion were consistently detected by P10-30 in the absence of other obvious abnormalities (data not shown), although the ventral expansion of the SVZ was not detected at this age.

Close examination of the cell morphology in the SVZ revealed that in contrast to wild-type control brains in which the SVZ was mainly composed of well-organized spindle-like progenitor cells with scant cytoplasm, the expanded SVZ in Pten $^{c K O}$ mice contained a central region of cells with large round nuclei interspersed with isolated progenitor cells, and with clusters of progenitor cells at the edge (Figure 2-3A). This expanded region of the SVZ and RMS was composed of Dcx+ and NeuN+ cells which 


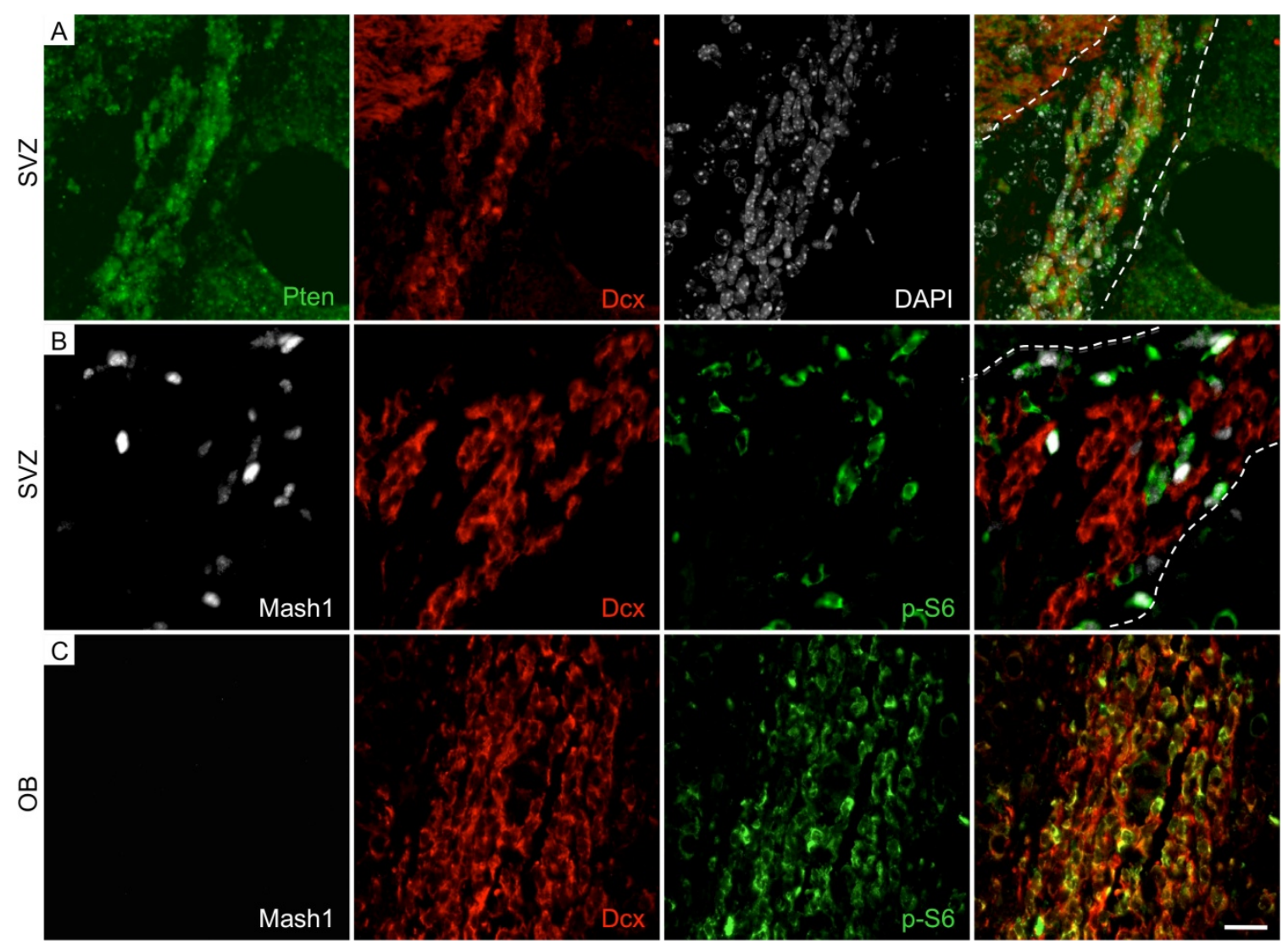

Figure 2-1. The PI3K-mTorc1 pathway was inactive in migrating SVZ neuroblasts, but activated in $O B$ neuroblasts.

IF staining in the SVZ (A and B) and OB (C) of coronal brain sections from 6-month old wild-type mice. (A) Pten (green) and Dcx (red) double IF labeling of the SVZ. Nuclei in the SVZ are shown with DAPI (white). Pten was expressed in Dcx+ cells in the SVZ (overlay of all channels in the far right panel). (B and C) Mash1 (white), Dcx (red) and pS6 (green) triple IF labeling of the SVZ (B) and OB (C), overlay shown in far right panels. p-S6 was expressed in Mash1+ transit amplifying cells but not Dcx + migrating neuroblasts in the SVZ (B) while in the OB, a substantial population of Dcx+ type A cells expressed p-S6 (C). The white dashed lines in the overlay mark the boundary of the SVZ in panels A and B. Scale bar: $20 \mu \mathrm{m}$. 
Figure 2-2. Nestin-CreER targeted neural stem/progenitor cells in the SVZ. (A) Schematic diagram of the Nestin-CreER transgene used to target neural stem/progenitor cells. (B and C) X-gal staining of sagittal (C and B left and center panels) or coronal (B, right panel) sections from Nestin-CreER;Rosa26R-LacZ reporter mice. (D) EYFP IF of SVZ from Nestin-CreER;Rosa26R-EYFP reporter mice. The mice were injected with vehicle or tamoxifen (TM) daily between P30-32 (B) or between P0-1 (C and D) and brains were collected 5 days after the first injection. The most uniform Cre activity was induced in the SVZ, RMS and the inner layer of the DG where neural stem/progenitors reside. The vast majority of cells in the SVZ were positive for the Cre reporters X-gal or EYFP. Cre reporter activity was also detected at low levels in scattered cells throughout other brain regions outside of neurogenic niches. This activity outside of the neural stem cell niche was more robust when Cre was induced in neonates $(C)$. (E) Gfap (red) + EYFP (green), Mash1 (red) + EYFP (green) and Dcx (red) + EYFP (green) double IF staining in the SVZ showed overlap of EYFP+ cells with the neural stem cells $($ Gfap +$)$, transit amplifying cells (Mash1+) or neuroblasts $(\mathrm{Dcx}+)$ in the far right panels. Scale bar: $\mathrm{D}=100 \mu \mathrm{m}, \mathrm{E}=10 \mu \mathrm{m}$. Nuclei were visualized with DAPI (blue). 


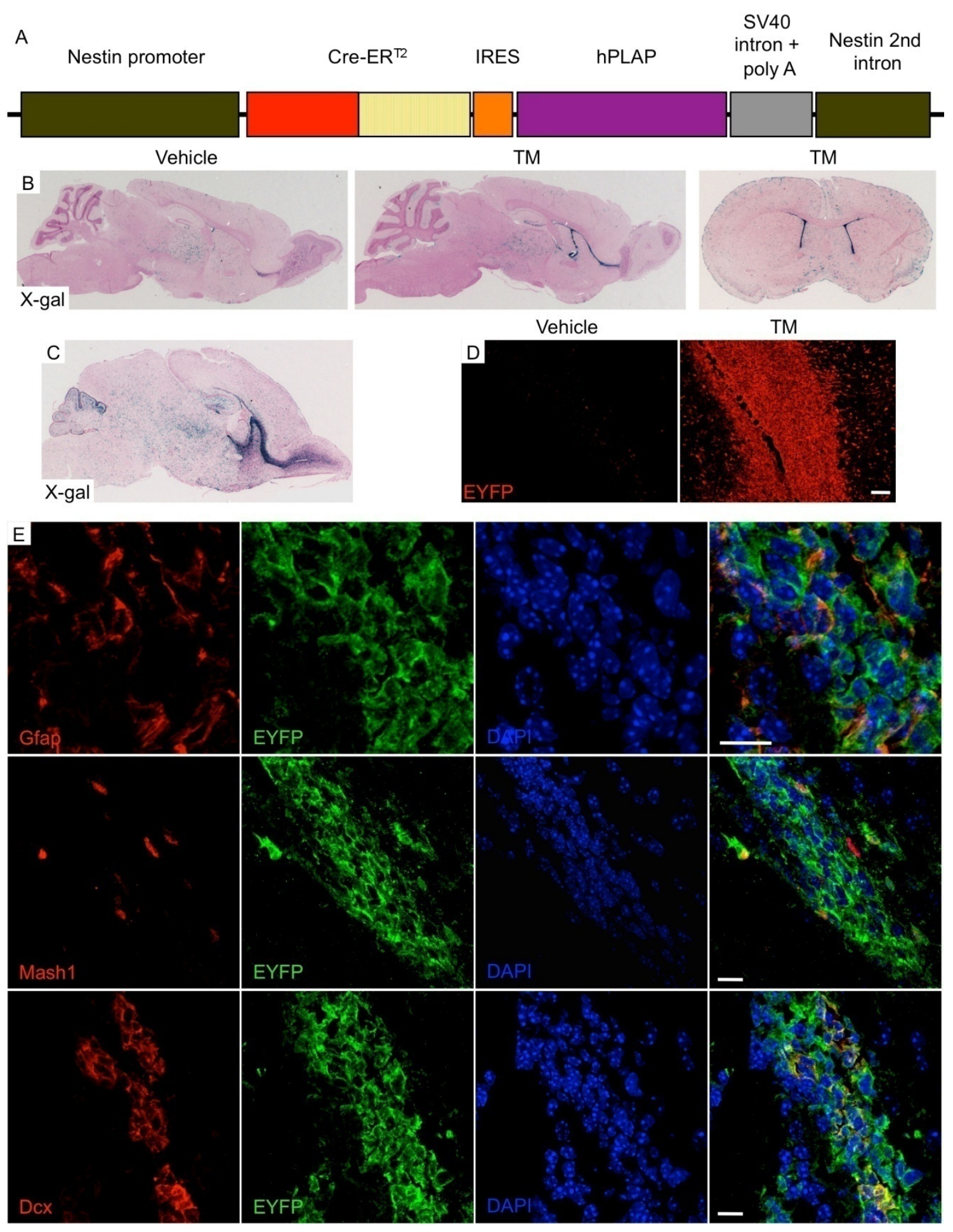



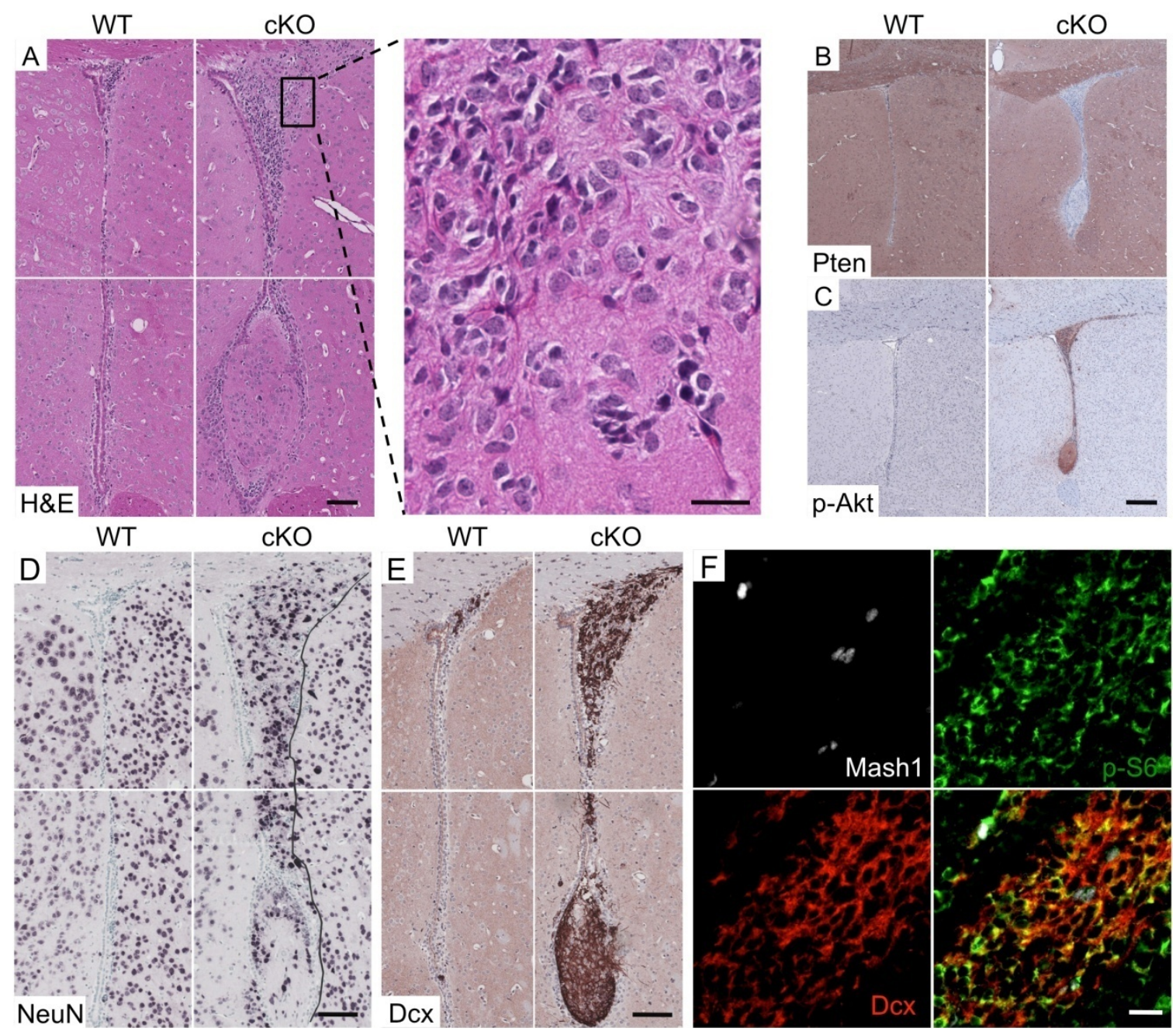

Figure 2-3. Postnatal Pten deletion caused ectopic differentiated neurons in an expanded SVZ.

Representative H\&E (A), IHC (B-E) and IF staining (F) of the SVZ from matched coronal brain sections of mice injected with TM on P30-32 and analyzed more than 70 days later $(\mathrm{n}=24)$. A, D and $\mathrm{E}$ upper and lower panels show dorsal and ventral SVZ, respectively. (A) H\&E staining showed that the Pten ${ }^{c K O}$ (cKO) SVZ was expanded compared to wild-type (WT). Black box on the cKO panel indicates the expanded SVZ region shown at higher magnification on the right panel. Scale bar: left panel $=100 \mu \mathrm{m}$, higher mag panel $=10 \mu \mathrm{m}$. (B-E) IHC staining of WT and cKO SVZ for Pten (B), p-Akt (C), NeuN (D) and Dcx (E). The expanded cKO SVZ was deficient for Pten, strongly positive for p-Akt and composed of NeuN+ and Dcx+ cells in contrast to the WT SVZ which had only thin layers of Dcx + cells and lacked NeuN + cells. In panel D, the NeuN+ cells adjacent to the wild-type SVZ were from surrounding striatum and the black line marks the edge of the expanded SVZ in cKO brain. Scale bar: B and C $=300 \mu \mathrm{m}, \mathrm{D}$ and $\mathrm{E}=100 \mu \mathrm{m}$. (F) Mash1 (white), p-S6 (green) and Dcx (red) triple IF labeling of the expanded cKO SVZ showed a substantial overlap of $\mathrm{p}-\mathrm{S} 6+$ and $\mathrm{Dcx}+$ cells in the right bottom panel. Scale bar: $20 \mu \mathrm{m}$. 


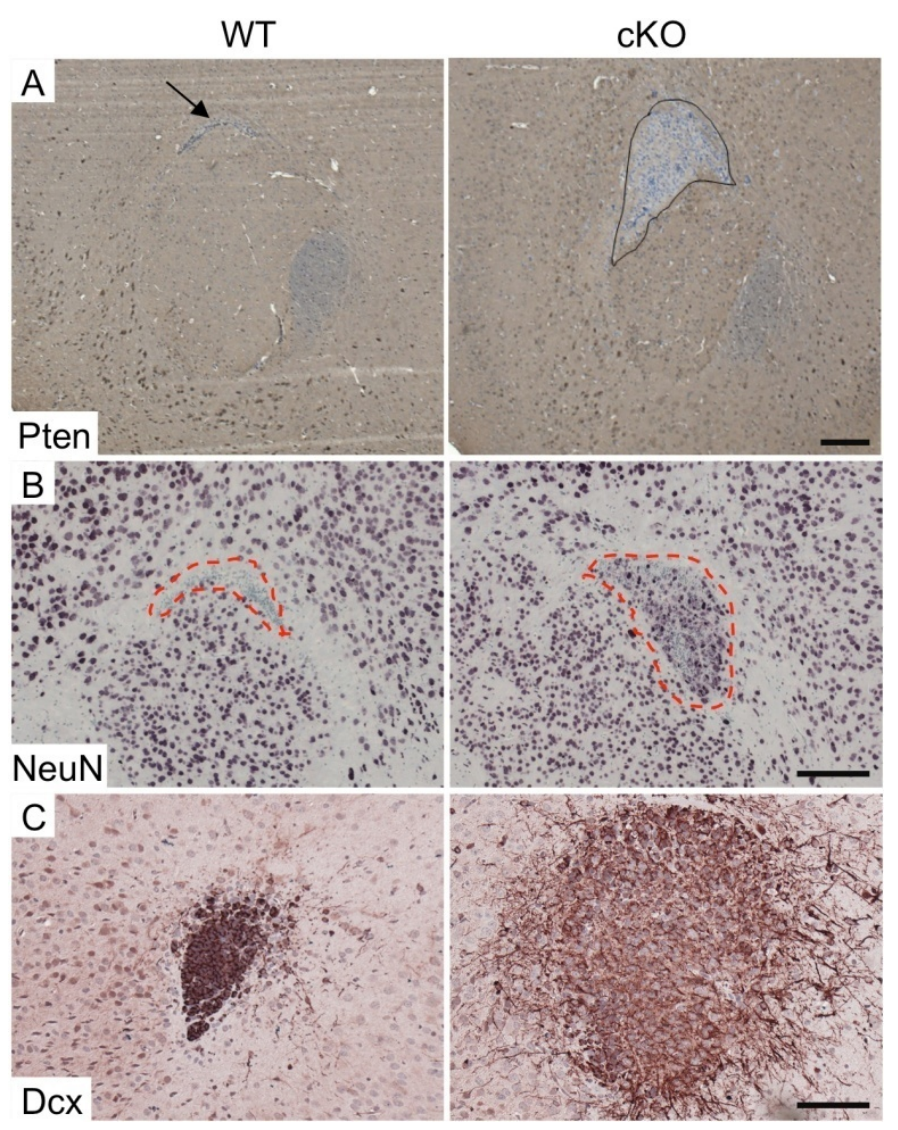

Figure 2-4. Expansion and ectopic neuronal differentiation in the RMS. IHC staining of the RMS from matched coronal brain sections of mice injected with TM on P30-32 and analyzed at 6 months of age (A and B) or mice injected with TM on P0-1 and analyzed at 18 days of age (C). (A) Pten IHC staining of both the WT and cKO RMS showed the cKO RMS was expanded and deficient for Pten. The arrow points to a thin layer of RMS in the WT whereas the solid black line outlines the cKO RMS. Scale bar: $200 \mu \mathrm{m}$. (B) NeuN IHC staining of both the WT and cKO RMS showed the cKO RMS was expanded and composed of NeuN+ cells, whereas the WT RMS was a thin layer devoid of NeuN+ cells. Red dashed lines outline both the WT and cKO RMS. Scale bar: $100 \mu \mathrm{m}$. (C) Dcx IHC staining of WT and cKO RMS showed expansion of the cKO RMS and formation of projections, whereas in the WT control, DCX expression was in a small rim tightly surrounding nuclei. Scale bar: $100 \mu \mathrm{m}$. 
mark migrating neuroblasts and differentiated neurons respectively (Figure 2-3D, E and Figure 2-4B, C). p-S6 staining in the Dcx+ neuroblasts of the Pten ${ }^{c K O} \mathrm{SVZ}$ (Figure 2-3F) shows aberrant activation of the PI3K pathway in contrast to the absence of $\mathrm{p}$-S6 expression in SVZ neuroblasts in wild-type mice (Figure 2-1B). The Dcx+ cells in the expanded SVZ and RMS showed evidence of neuronal differentiation including abnormal formation of projections (Figure 2-3E and Figure 2-4C), and Map2 expression, which marks dendrites in polarized neurons (Figure 2-5). The expression of Dcx decreased and NeuN expression increased in the ectopic cells as mice aged from 150 DPI to 300 DPI (data not shown). The expression of mature neuronal markers in Dcx + cells in the SVZ indicates ectopic differentiation of neuroblasts that normally remain undifferentiated until they reach the OB. Furthermore, the ectopic cells also expressed calretinin (Figure 2-5), a marker expressed in mature GABAergic interneurons of the OB arising from neuroblasts that migrate from the SVZ through the RMS to populate the GCL and the periglomerular cell layer of the OB (Kohwi et al. 2005; Kohwi et al. 2007; AlvarezBuylla et al. 2008).

Consistent with a failure of migrating neuroblasts to reach the $\mathrm{OB}$, the diameter of the terminal RMS at the core of the OB and the cell density in the GCL were markedly reduced in $\mathrm{Pten}^{c K O}$ mice (Figure 2-6 and Figure 2-7A, C).

\section{Pten loss did not induce substantial changes in proliferation or survival in the expanded Pten $^{c K O} \mathrm{SVZ}$}

To determine how loss of Pten affected the proliferation in the intact SVZ in vivo, we used both Ki67 IHC and short-term BrdU labeling, which gave consistent results (Figure 2-8A and data not shown). Because the cell numbers in the SVZ of Pten ${ }^{c K O}$ mice were increased due to the presence of ectopic post-mitotic differentiated cells, considering the percentage of proliferating cells would underestimate the proliferation capacity of progenitors in the Pten ${ }^{c K O}$ SVZ. Therefore, we counted the absolute numbers of Ki67+ cells from matched SVZs of both wild-type and Pten ${ }^{c K O}$ mice. There was no statistically significant difference in the proliferation rate in the Pten ${ }^{c K O} \mathrm{SVZ}$ compared to control littermates (Figure 2-8A, C).

Activated PI3K signaling can also enhance cell survival, therefore we used active caspase 3 and TUNEL staining to determine if Pten loss altered apoptosis in the SVZ (Figure 2-9A). The endogenous level of apoptosis was very low, and not significantly different in wild-type and Pten ${ }^{c K O}$ SVZ. Consistent with the resistance of differentiated neurons to radiation induced death (Frappart and McKinnon 2008), the expanded portion of the Pten $^{c K O}$ SVZ containing differentiated neurons as well as the parenchyma surrounding the SVZ were resistant to irradiation-induced apoptosis (Figure 2-9B). However, the progenitor cells of both wild-type and $\operatorname{Pten}^{c K O}$ underwent apoptosis 5 hours after exposure to $4 \mathrm{~Gy}$ of irradiation. Therefore, Pten-deficient neuroblasts did not show enhanced survival. 


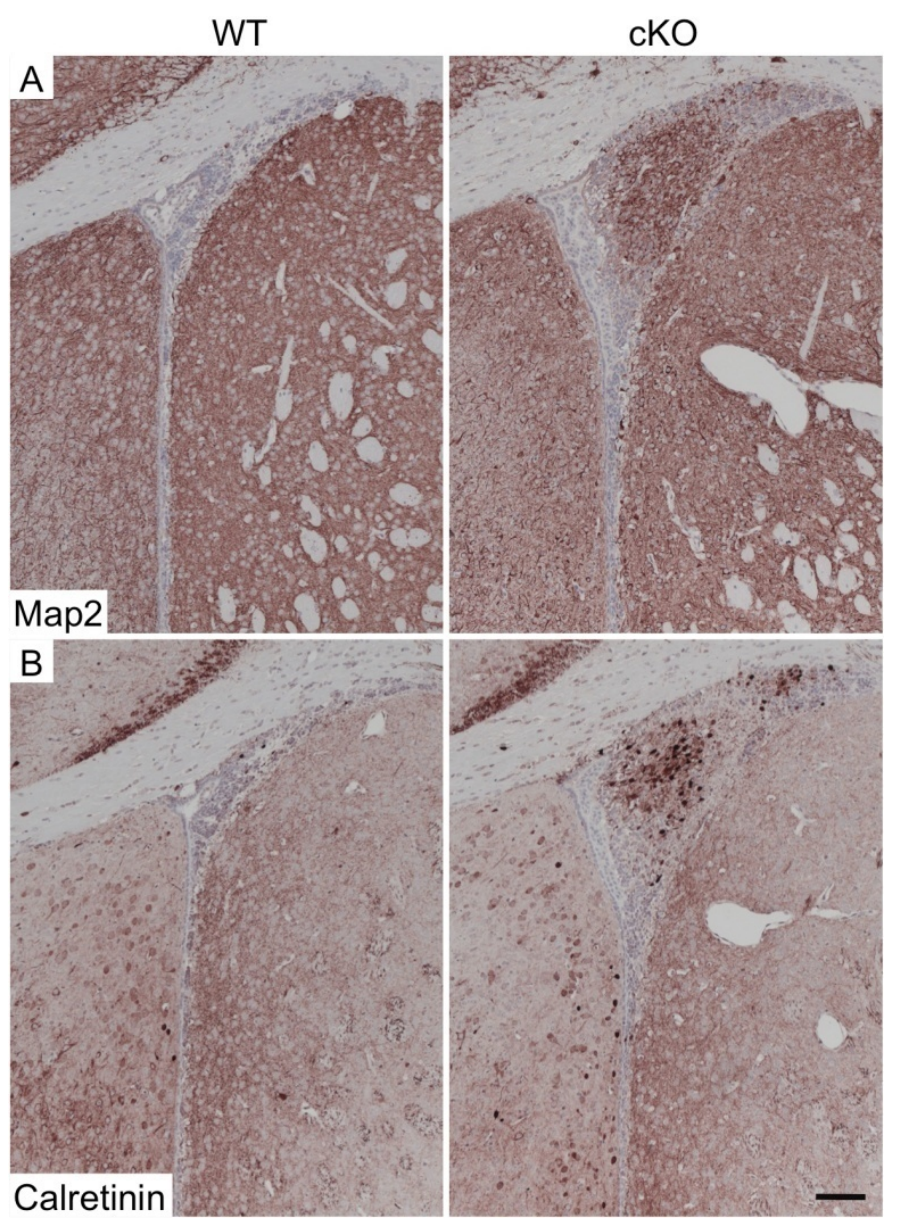

Figure 2-5. Ectopic cells in the expanded cKO SVZ expressed markers of mature neurons.

Map2 (A) and Calretinin (B) IHC staining of the SVZ from matched coronal brain sections of WT (left) or cKO (right) mice injected with TM on P0-1 and analyzed at 18 days of age showed strong expression of these markers of neuronal differentiation in the expanded cKO SVZ. Scale bar: $100 \mu \mathrm{m}$. 


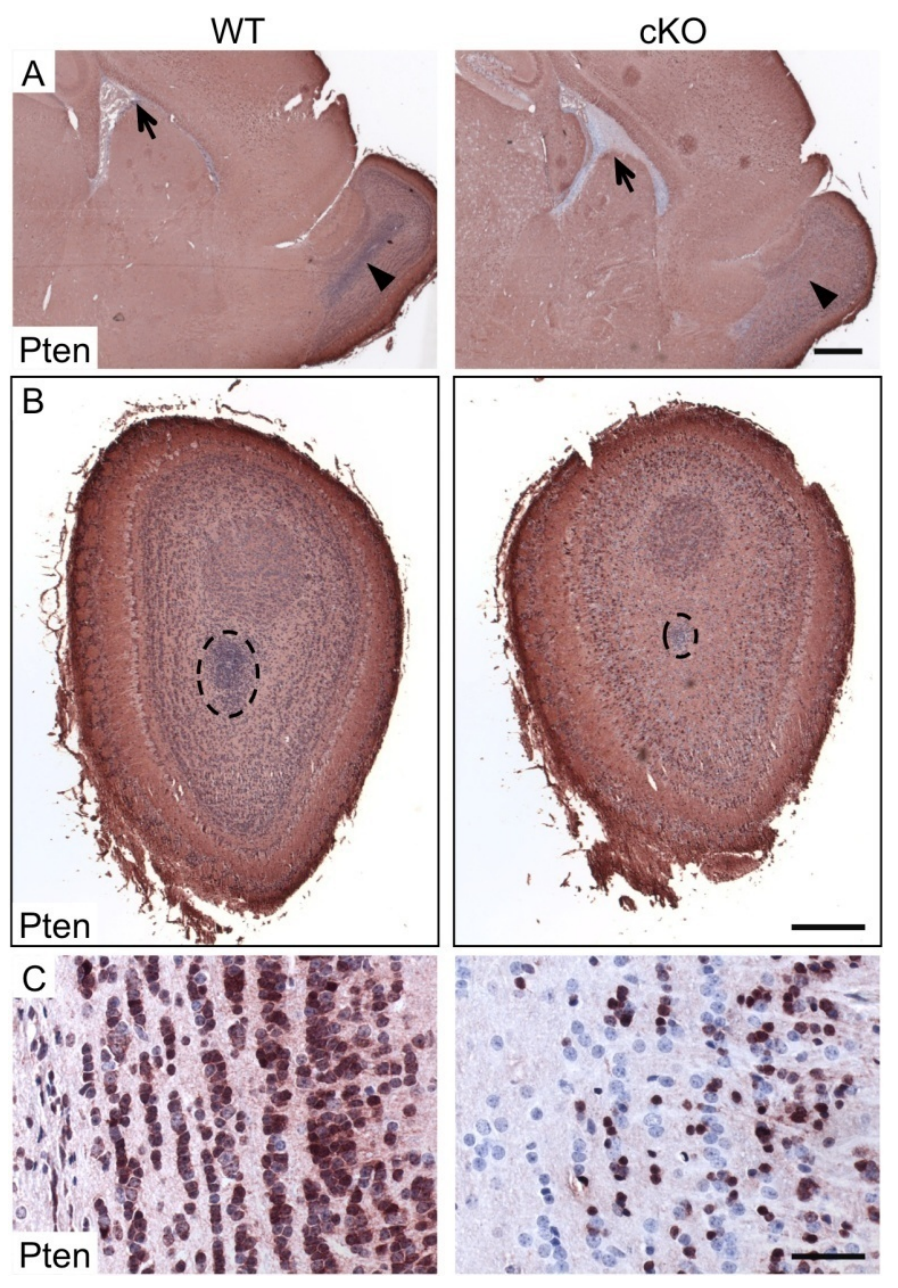

Figure 2-6. Reduced diameter of the terminal RMS and decreased granule cell density in the PtencKO OB.

Pten IHC staining from matched saggital brain sections of the forebrain (A) or coronal brain section of the OB (B and C) from WT and cKO mice injected with TM on P0-1 and analyzed at P18 (A and B) or injected with TM on P30-32 and analyzed at 11 months of age (C). The cKO SVZ was expanded and deficient for Pten (indicated by arrows in panel A). The diameter of the terminal RMS in the $\mathrm{cKO} O \mathrm{OB}$ was diminished (indicated by arrowheads in panel $A$ and dashed circles in panel B). The cell density of the GCL in the cKO OB was reduced (C). Scale bar: $A=500 \mu \mathrm{m}, \mathrm{B}=300 \mu \mathrm{m}, \mathrm{C}=100 \mu \mathrm{m}$. 

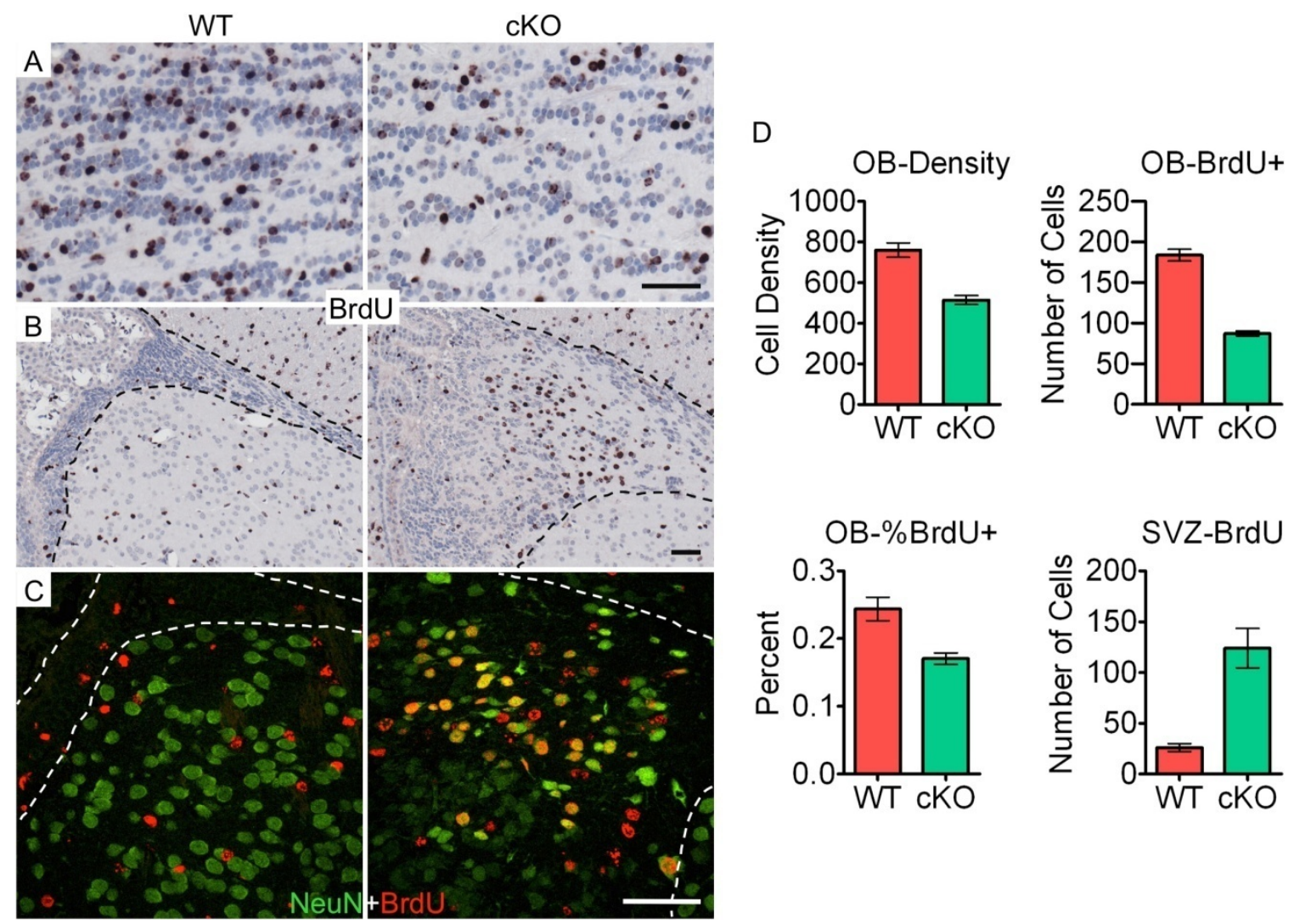

\section{Figure 2-7. Pten $^{c K O}$ neuroblasts terminated tangential migration prematurely in} the SVZ.

Immuno-staining (A-C) and quantification of BrdU+ cells (D) of matched saggital brain sections from WT (left) and cKO (right) mice. Cre activity was induced by tamoxifen injection at $\mathrm{P} 0$ and $1, \mathrm{BrdU}$ was injected on $\mathrm{P} 4,5$, and 6 , and tissue was analyzed at P18. (A and B) BrdU IHC staining. (A) In the OB, there were reduced numbers of BrdU+ cells in the granule cell layer of the cKO OB compared to WT. (B) In the SVZ, there was a significant accumulation of BrdU+ cells in the expanded cKO SVZ. (C) BrdU (red) and NeuN (green) double IF labeling of the SVZ from WT and cKO mice showed a substantial number of BrdU+ cells in the expanded cKO SVZ were $\mathrm{NeuN}+$, while there were no NeuN+ cells in the WT SVZ. Black dashed lines in panel B and white dashed lines in panel C mark the boundary of the SVZ. Scale bar: $50 \mu \mathrm{m}$. (D) Quantification of the granule cell density in OB (OB-Density, $p=0.001$ ), BrdU+ cell in the OB (OB$\mathrm{BrdU}+, \mathrm{p}<0.0001)$ and the percentage of $\mathrm{BrdU}+$ cells in the $\mathrm{OB}(\mathrm{OB}-\% \mathrm{BrdU}+, \mathrm{p}=0.008)$ were all significantly reduced in the GCL of the $\mathrm{cKO} O \mathrm{OB}$, whereas the total number of BrdU+ cells in the cKO SVZ (SVZ-BrdU, $p=0.003$ ) was markedly increased $(n=4$, for each genotype). 

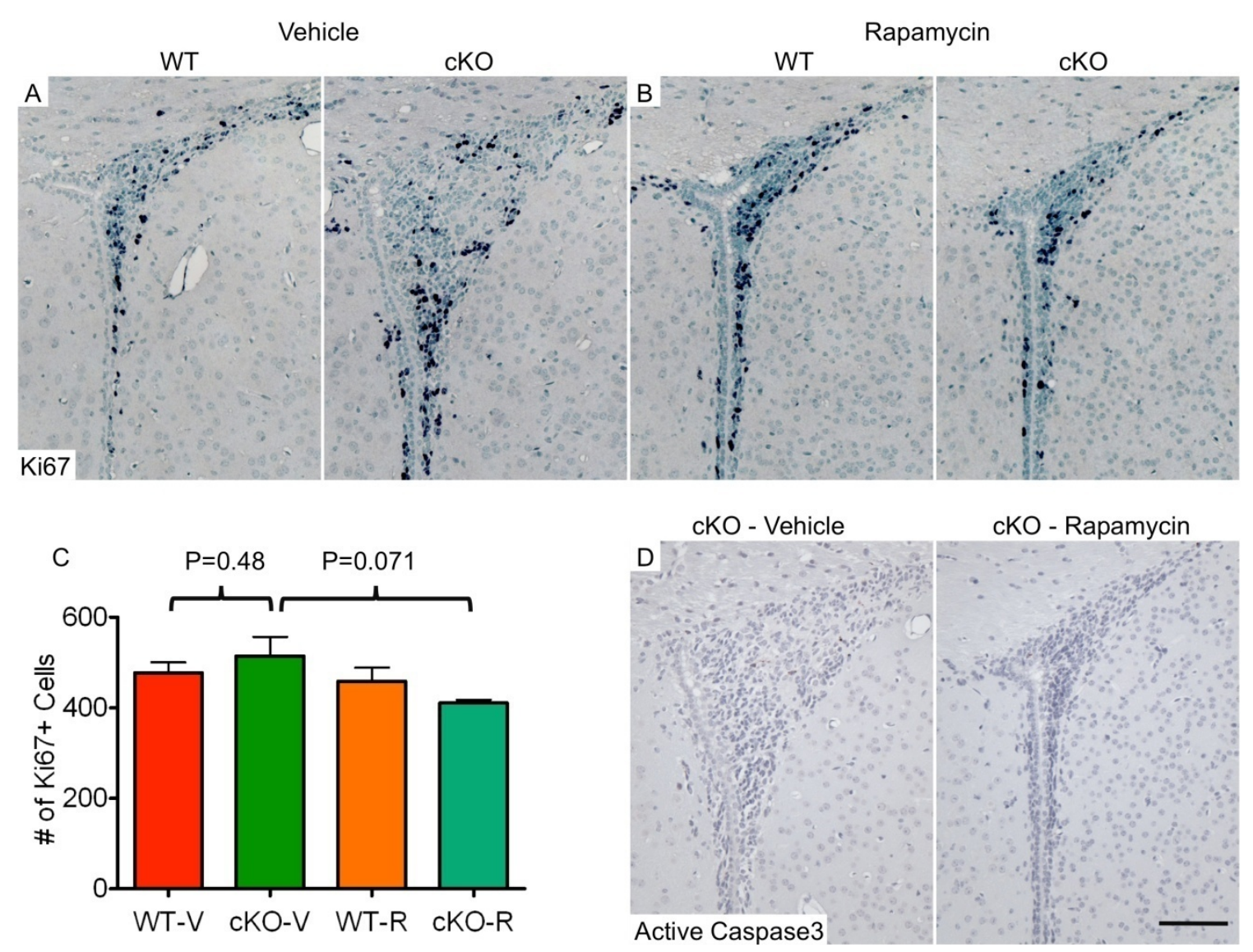

Figure 2-8. $\quad$ mTorc1 inhibition did not affect proliferation or apoptosis in the SVZ. Ki67 IHC staining in the SVZ of matched coronal brain sections from WT or cKO mice treated with $(\mathrm{A})$ vehicle or $(\mathrm{B})$ rapamycin. Mice were treated with rapamycin or vehicle from P8-P31, Cre activity was induced by TM injection at P11 and P12, and tissue was analyzed at P31. Scale bar: $100 \mu \mathrm{m}$. (C) Quantification of Ki67+ cells in the SVZ showed no significant difference between vehicle-treated control and $\mathrm{cKO}$ or between vehicle and rapamycin-treated cKO. (D) active Caspase 3 IHC staining of the SVZ from vehicle or rapamycin-treated cKO mice. There were few active Caspase $3+$ cells in either vehicle or rapamycin-treated cKO SVZ, and no difference was detected. Scale bar: $100 \mu \mathrm{m} . \mathrm{n}=3$ mice per genotype. 

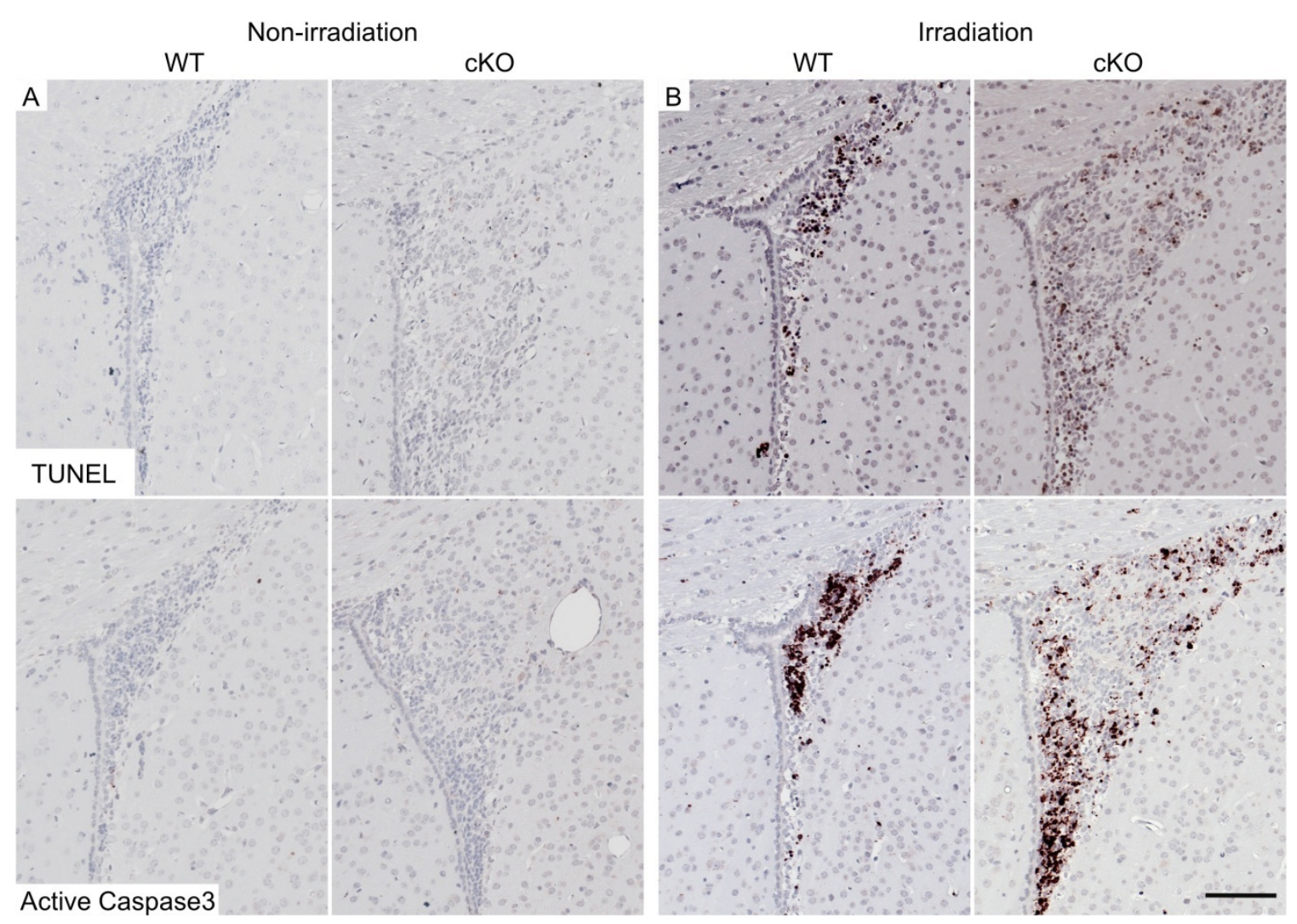

Figure 2-9. Pten ${ }^{c K O} \mathrm{SVZ}$ neural progenitors were sensitive to irradiation and showed no change in apoptosis compared to control mice.

(A) TUNEL (top panels) and active caspase 3 (bottom panels) IHC staining of the SVZ in matched coronal sections from mice injected with TM on P0-1 and analyzed at 18 days old. The endogenous levels of apoptosis were very low in the SVZ and no significant difference in apoptosis was detectable between untreated WT and cKO mice. (B) The same IHC staining as in panel A. Mice were irradiated with 4 Gy and tissue collected 5 hrs later. The progenitor cells in both WT and cKO SVZ underwent apoptosis after irradiation whereas the parenchyma surrounding the SVZ and the expanded part of the cKO SVZ were resistant to irradiation-induced apoptosis. Scale bar: $100 \mu \mathrm{m}$. 


\section{Pten $^{\text {cKo }}$ neuroblasts terminated tangential migration prematurely in the SVZ and RMS}

To determine if the ectopic neurons arose from proliferative cells in the SVZ that underwent premature termination of tangential migration along the SVZ-RMS-OB, we used BrdU birth dating. Pten deletion was induced by TM injection in newborn mice. Four days after TM induction, to allow sufficient time for Pten deletion and loss of Pten protein, BrdU was injected to label proliferating cells in the SVZ. As expected, the majority of labeled cells in control mice migrated into the GCL of the OB by 15 days after the BrdU pulse (Petreanu and Alvarez-Buylla 2002) (Figure 2-7A). In comparison, there was a significant accumulation of BrdU+ cells in the expanded SVZ and greatly reduced numbers of BrdU+ cells, and reduced granule cell density in the OB GCL in the Pten $^{c K O}$ mice (Figure 2-7A,B,D). A high percentage of BrdU+ cells in the expanded SVZ showed neuronal morphology and expressed NeuN, indicating that the ectopic differentiated neurons arose from previously proliferating cells in the SVZ (Figure 2-7C). In control mice, there were few BrdU+ cells remaining in the SVZ, and none of them had neuronal morphology or expressed NeuN. They may correspond to slowcycling neural stem cells or local proliferating glia (Figure 2-7C).

\section{Inhibition of mTorc1 rescued the SVZ-RMS expansion in $\operatorname{Pten}^{c K O}$ brain}

To determine if inhibition of a downstream effector in the aberrantly activated PI3K pathway could prevent the early termination of tangential migration and ectopic differentiation of Pten ${ }^{c K O}$ neuroblasts in the SVZ, we treated the mice with rapamycin, an inhibitor of mTorc1 activity. We previously showed that rapamycin required several days of administration to maximally block mTorc1 signaling in brain (Kwon et al. 2003). Therefore, we pre-treated the mice daily with rapamycin for three days, then induced the deletion of Pten with TM at P11-12 and continued rapamycin treatment until P31. Rapamycin treatment completely prevented the expansion of the SVZ in Pten ${ }^{c K O}$ mice (Figure 2-10A), although the SVZ remained Pten-null in rapamycin treated mice (Figure 2-10B). Rapamycin selectively blocked mTorc1 activity, as shown by lack of p-S6, a downstream indicator of mTorc1 activity (Figure 2-10C) without altering levels of p-Akt in the Pten-deficient SVZ (Figure 2-10D). Neither apoptosis nor proliferation was affected by rapamycin treatment (Figure 2-8).

\section{Ex vivo time-lapse live imaging showed $\operatorname{Pten}^{c K O}$ neuroblasts had normal directional migration with increased speed}

To understand how Pten loss affected the movement of tangentially migrating neuroblasts, we used time-lapse live imaging on acute brain slices. We transfected the expression construct $p C A G-C r e$ into the SVZ by in vivo electroporation at age $\mathrm{P} 2$ and visualized transfected cells by expression of the EYFP Cre reporter in $R 26 R-$

EYFP;Pten ${ }^{w t / w t}$ (wild-type) or R26R-EYFP;Pten ${ }^{\text {loxP/loxP }}\left(\right.$ Pten $\left.^{c K O}\right)$ mice. Neuroblast migration was imaged with two-photon microscopy at the "elbow" of the RMS, at the 
Figure 2-10. mTorc1 inhibition rescued the expansion of Pten ${ }^{c K o} \mathrm{SVZ}$.

Representative IHC staining for Dcx (A), Pten (B), pS6 (C), and pAkt (D) in the SVZ of matched coronal brain sections from vehicle or rapamycin treated WT or $\mathrm{cKO}$ mice. Mice were treated with rapamycin or vehicle from P8-P31, Cre activity was induced by TM injection at P11 and P12, and tissue was analyzed at P31. Rapamycin completely rescued the enlargement of cKO SVZ to restore the size and morphology seen in WT mice ( $\mathrm{n}=4$ in each group). The $\mathrm{SVZ}$ of rapamycin treated cKO mice remained Pten-null (B) and continued to show elevated pAkt (D), but showed suppression of pS6 (C), a downstream indicator of mTorc 1 activity. Scale bar: $50 \mu \mathrm{m}$. 


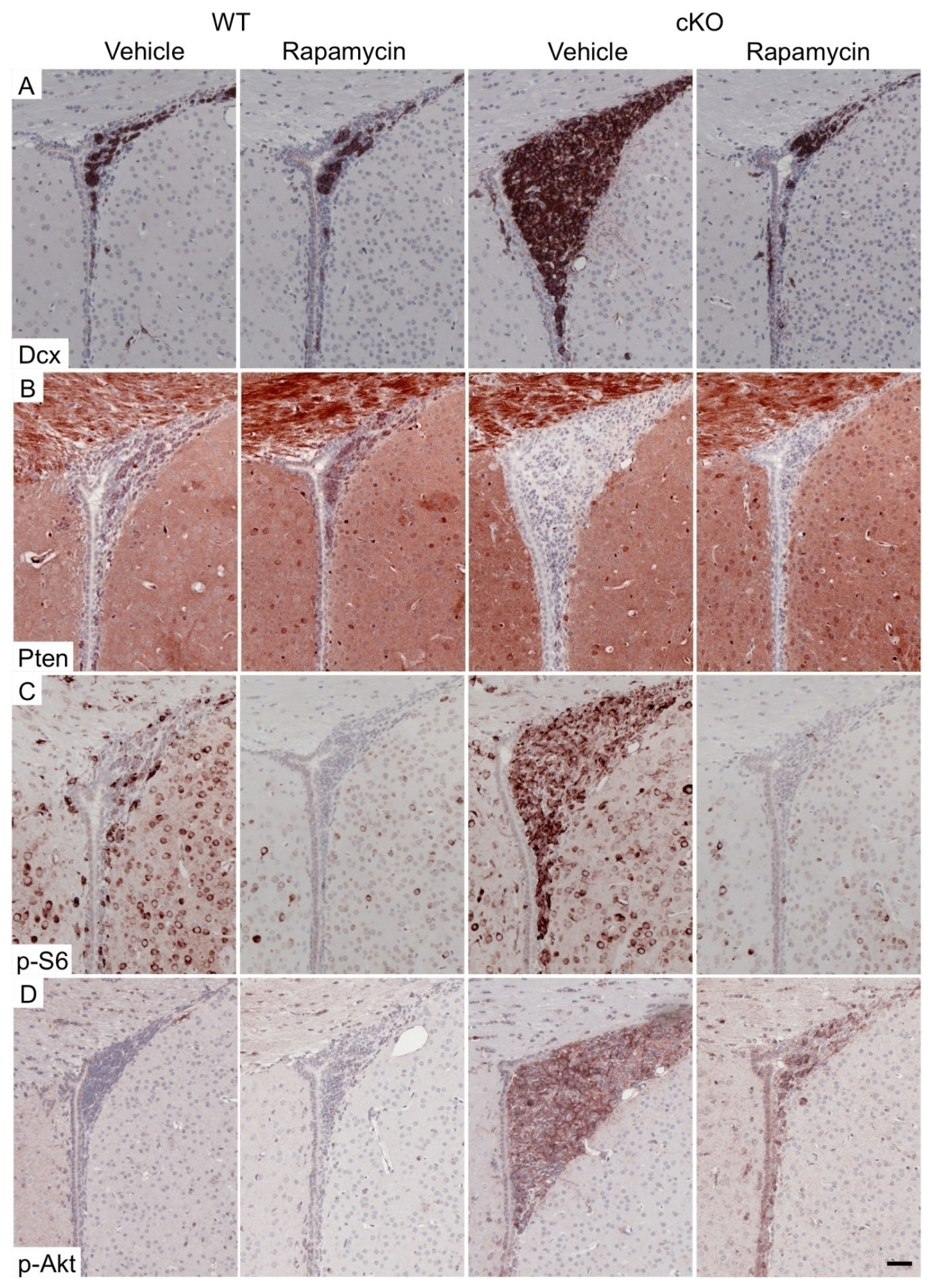


region where the RMS curves from ventral towards rostral migration halfway between the SVZ and the OB (see Figure 2-11 C for the location) at age P19-20. The majority of EYFP + cells in the wild-type RMS showed a bipolar morphology and migrated effectively. In contrast, there were two distinct populations of EYFP+ cells in the Pten $^{c K O}$ RMS (Figure 2-12A). One population maintained bipolar morphology and migrated normally as in the wild-type control, while another population lost polarity and showed only local non-directional movement. Interestingly, the cells that had stopped directional migration showed active spontaneous membrane ruffling, which distinguished them from completely static cells seen at very low frequency in both the wild-type and Pten $^{c K O}$ RMS (Supplementary movies A-1 and A-2). The average speed of all EYFP+ cells in the $P \operatorname{ten}^{c K O}$ RMS (mean: $0.0096 \mu \mathrm{m} / \mathrm{s}$ ) was significantly slower than that of the wild-type (mean: $0.0143 \mu \mathrm{m} / \mathrm{s})\left(\mathrm{p}<10^{-19}\right)$. However, the average speed of bipolar migrating neuroblasts in the Pten ${ }^{c K O}$ RMS (mean: $0.0173 \mu \mathrm{m} / \mathrm{s}$ ) were slightly but significantly faster than the wild-type (mean: $0.0156 \mu \mathrm{m} / \mathrm{s})(\mathrm{p}=0.006)$ (Figure 2-12B, C). The Pten ${ }^{c K O}$ bipolar migrating neuroblasts moved effectively in the appropriate caudal to rostral direction, as their endpoint speeds, a representation of directional movement, were slightly but significantly greater than the wild-type (mean $0.0145 \mu \mathrm{m} / \mathrm{s}$ and $0.0131 \mu \mathrm{m} / \mathrm{s}$, respectively $(\mathrm{p}=0.047)$. Accordingly, a substantial number of Pten-deficient neuroblasts were able to migrate to the OB (Figure 2-11). Both the EYFP+ bipolar and round nonpolar cells in the RMS expressed Dcx, indicating that they were migrating neuroblasts or neuroblasts that stopped migration recently (Figure 2-13). These data indicate that Ptendeficient neuroblasts do not have an intrinsic defect that compromises migration.

\section{Discussion}

\section{Pten function in neuronal migration and differentiation}

Multiple conditional knock-out models have shown that Pten deletion during development results in incomplete neuronal migration causing disruption of the laminar structure in cerebral cortex and cerebellum. The accumulation of ectopic differentiated $\mathrm{Pten}^{c K O}$ neurons in the SVZ and RMS observed in the present study is consistent with defects in tangential migration, similar to the failure of radial glia-guided migration in the cerebral cortex and cerebellum observed in previous models (Backman et al. 2001; Groszer et al. 2001; Kwon et al. 2001; Marino et al. 2002; Yue et al. 2005). SVZ expansion associated with premature termination of tangential migration was also observed in mouse models knocking out PSA-NCAM (Tomasiewicz et al. 1993; Ono et al. 1994), serum response factor (Alberti et al. 2005), ApoER2/VLDL or Dab1 (Andrade et al. 2007), which might suggest an epistatic relationship between them and Pten.

PTEN and the PI3K-Akt-mTorc1 pathway have been implicated in cell migration regulation in multiple contexts. Mechanistically, this has been mostly clearly demonstrated in Dictyostelium discoideum where subcellular localization of Pten and PI3K to the trailing and leading edge of the cell, respectively, established a PIP3 gradient 


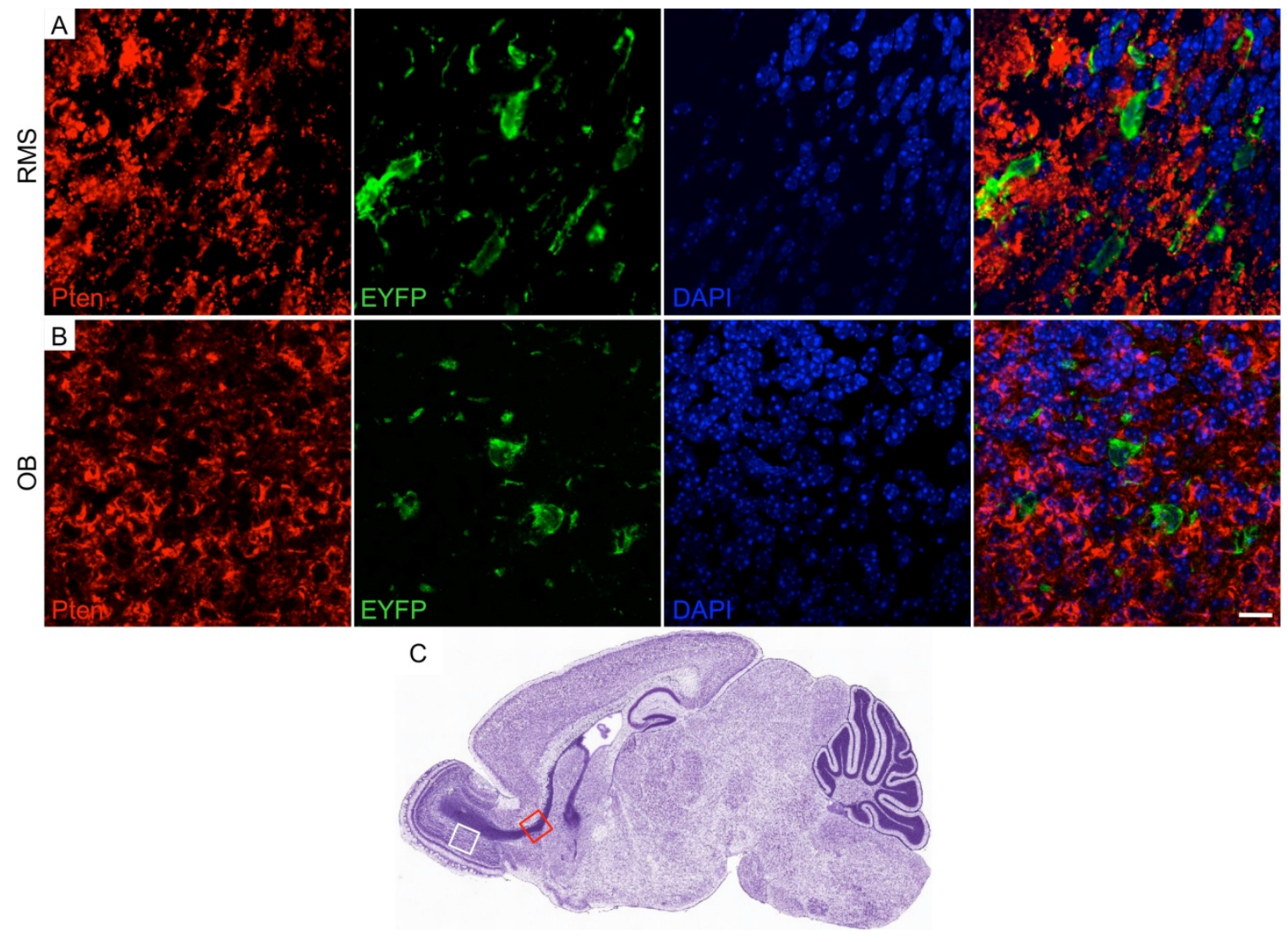

Figure 2-11. EYFP+ cells in the Pten ${ }^{\text {cKo }}$ RMS and OB were Pten-null.

Pten $^{\text {floxflox }}$ (cKO) mice carrying a ROSA-lox-STOP-lox-EYFP allele were transfected with Cre by in vivo electroporation as described for Figure 2-12. EYFP and Dcx double IF labeling of the cKO RMS elbow from littermate mice electroporated at the same time as those used for in vivo time lapse imaging showed that both the arrested non-polar cells (arrowheads) and the elongated migrating cells (arrow) in the cKO RMS were positive for Dcx (overlay in the bottom right panel). Scale bar: $100 \mu \mathrm{m}$. 

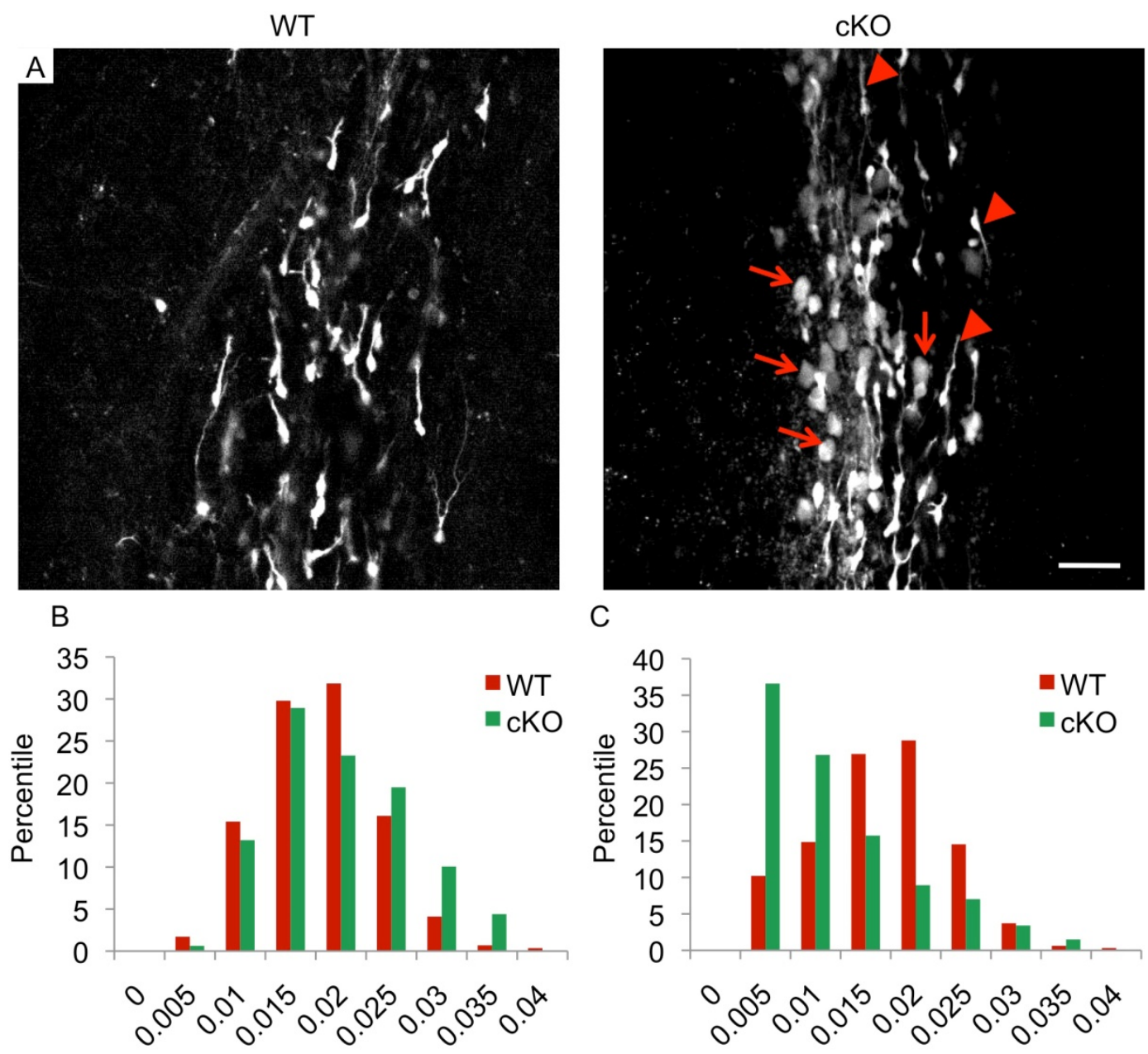

C

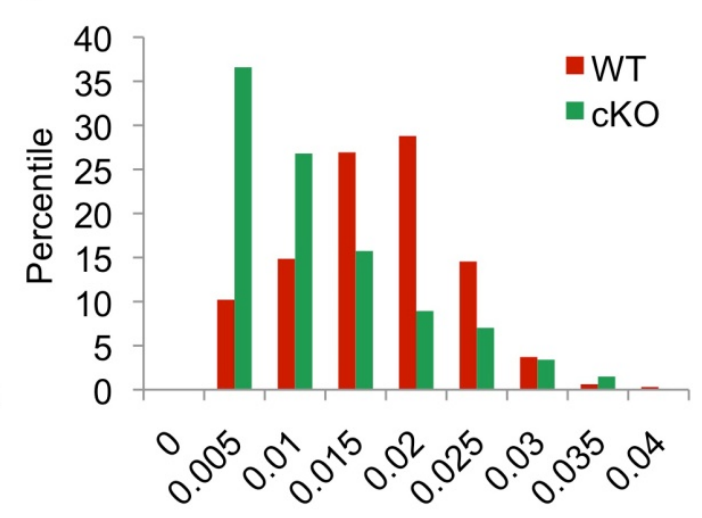

Avg Speeds of bipolar cells $(\mu \mathrm{m} / \mathrm{s})$

Avg Speeds of all cells $(\mu \mathrm{m} / \mathrm{s})$

Figure 2-12. Ex vivo time-lapse live imaging showed Pten $^{c K O}$ neuroblasts had normal directional migration with faster speed.

(A) Representative static EYFP images corresponding to the time-lapse migration movies from the RMS (supplemental). A Cre expression construct was transfected by in vivo electroporation at P2 into the SVZ of WT or Pten ${ }^{\text {floxflox }}$ (cKO) mice carrying a ROSAlox-STOP-lox-EYFP allele. Acute brain slices were prepared at P19-20, and cells in which Cre-mediated recombination occurred were visualized by EYFP reporter expression using two-photon microscopy. The vast majority of WT neuroblasts had bipolar morphology and were migrating. There were two distinct populations of Pten ${ }^{c K O}$ (cKO) neuroblasts; cells with elongated bipolar morphology and normal migration (indicated by arrowheads), and cells with large rounded morphology that showed only local non-directional movement (indicated by arrows). Scale bar: $50 \mu \mathrm{m}$. (B and C) Quantification and histogram of average speeds for bipolar migrating EYFP+ cells (B) or all EFYP+ cells (C) from WT (red bars) and cKO RMS (green bars). $n=3$ mice per genotype. 


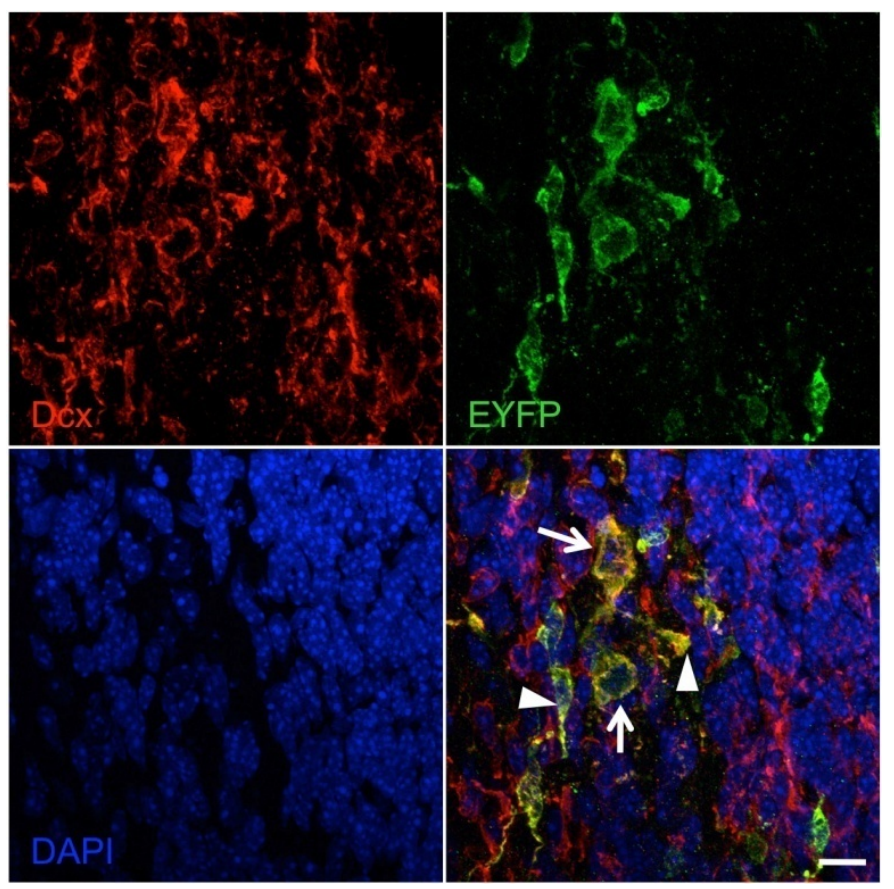

Figure 2-13. The arrested EYFP+ cells in the Pten $^{c K O}$ RMS were in the neuronal lineage.

Pten $^{\text {floxflox }}$ (cKO) mice carrying a ROSA-lox-STOP-lox-EYFP allele were transfected with Cre by in vivo electroporation as described for Figure 2-12. EYFP and Dcx double IF labeling of the cKO RMS elbow from littermate mice electroporated at the same time as those used for in vivo time lapse imaging showed that both the arrested non-polar cells (arrowheads) and the elongated migrating cells (arrow) in the cKO RMS were positive for Dcx (overlay in the bottom right panel). Scale bar: $100 \mu \mathrm{m}$. 
that was required for directional migration and chemotaxis (Funamoto et al. 2002; Iijima and Devreotes 2002). Deletion of the Akt homolog PkbA, rescued the cytokinesis and chemotaxis defects in Pten deficient Dictyostelium (Tang et al. 2011). In mammalian cells, the PI3K pathway can regulate cell motility and migration through Akt activation as well as through Akt-independent mechanisms such as RAC1/CDC42 signaling (Kolsch et al. 2008). In addition, overexpression of wild-type PTEN inhibited migration of human glioma cell lines in an in vitro wound healing assay, and this effect was dependent on the protein phosphatase activity of PTEN independent of PI3K signaling (Raftopoulou et al. 2004).

Unexpectedly, we found that Pten-null neuroblasts in the RMS had normal directional movement with faster speed using two-photon time-lapse microscopy of ex vivo slice cultures that maintain the physiological context for neuronal migration. Postnatal deletion of Pten divided the RMS neuroblasts into two distinct populations; one subset maintained their bipolar morphology and had normal directional migration and the other population lost polarity and stopped migration. This non-polar morphology, in association with increased frequency of spontaneous protrusions of the plasma membrane, was reminiscent of Pten-null Dictyostelium. However, the population of $P t e n^{c K O}$ neuroblasts that maintained bipolar morphology continued moving normally, demonstrating that Pten was not required for directional migration. This is consistent with the observation that some Pten-null cells reach the $\mathrm{OB}$ in $\operatorname{Pten}^{c K O}$ mice. A previous study in which Pten was deleted in Gfap-expressing neural stem cells also showed that Pten-deficient neurons were capable of reaching the olfactory bulb. This study also noted an expansion of Dcx + cells in the SVZ and RMS, although they did not analyze expression of mature neuronal markers in these cells (Gregorian et al. 2009). Postnatal deletion of Pten in cerebellar granule neurons also showed that a subset of Pten-null granule cells migrated to the proper position in the internal granule layer, while many were ectopically located in the molecular layer and at the pial surface (Backman et al. 2001; Kwon et al. 2001). Failed migration of Pten-deficient cerebellar neurons has been suggested to be secondary to defects in the glia guiding their migration (Yue et al. 2005). However, the in vivo electroporation approach used in the present study targeted small numbers of cells and identified stationary Dcx + neuroblasts without Cre-mediated deletion in other surrounding cell types. The results in the RMS are consistent with cell autonomous defects leading to ectopic positioning.

Normal directional migration of Pten-null neuroblasts is consistent with the finding that directional chemotaxis can still occur in Dictyostelium in the absence of a PIP3 gradient (Hoeller and Kay 2007). Additionally, several studies showed that Pten was not essential in directional migration and chemotaxis of mammalian leukocytes (Lacalle et al. 2004; Ferguson et al. 2007; Nishio et al. 2007; Subramanian et al. 2007; Heit et al. 2008). Pten deficient neutrophils showed an increased migration speed similar to the neuroblasts in the our study (Subramanian et al. 2007). Thus, there are likely contextdependent signals driving the effect of Pten and PI3K signaling on migration.

Pten-deficient neuroblasts demonstrate an "all or none" phenotype of normal directional migration or abnormal morphology associated with arrested migration. Taken 
together, the data suggest that the ectopic localization of neurons is more likely attributed to premature differentiation and secondary loss of migration ability rather than intrinsic defects in migration. Indeed, the PI3K-Akt-mTorc1 pathway has been shown to play an important role in several crucial neuronal differentiation processes including neuronal polarity, axon guidance, dendrite arborization and spine morphogenesis (Campbell and Holt 2001; Jaworski et al. 2005; Kumar et al. 2005; Tavazoie et al. 2005; Chadborn et al. 2006; Kwon et al. 2006; Wildonger et al. 2008; Chow et al. 2009).

\section{PI3K-Akt-mTorc1 pathway regulation in the SVZ-RMS-OB}

The PI3K-Akt-mTorc1 pathway is dynamically regulated through the different developmental stages of neuronal lineage along the SVZ-RMS-OB. From birth in the SVZ to differentiation and integration into the local circuit of the $O B$, neural stem cells progress in phases to transit amplifying progenitors, migrating neuroblasts and ultimately terminally differentiated mature neurons. Accordingly, cell signaling pathways must be modulated to meet the varying requirements of these differentiation states or to induce transition between consecutive phases. In this study, we found that the PI3K-Akt-mTorc1 pathway is active in the transit amplifying cells and inactive in the migrating neuroblasts until activated again in the OB. Consistent with these findings, previous studies also found high expression of Egfr, a potent PI3K-Akt-mTorc1 pathway activating receptor, in the transit amplifying progenitors but not in the migrating neuroblasts in the SVZ. The abundant free ribosomes found in transit amplifying progenitors is consistent with activation of mTorc1 signaling (Doetsch et al. 2002; Kim et al. 2009; Kokovay et al. 2010).

Upregulation of the PI3K-Akt-mTorc1 pathway may act to drive proliferation of transit amplifying progenitors, while inactivation of PI3K-Akt-mTorc1 signaling through Pten expression may play a role in the transition to migrating neuroblasts which exit the cell cycle. In support of this theory, enhanced Egfr signaling in vivo expands the proliferative pool, arrests neuronal production (Doetsch et al. 2002; Aguirre et al. 2010) and negatively regulates migration in the SVZ (Kim et al. 2009). In our system, Pten deletion did not have a substantial effect on proliferation. The magnitude of PI3K signaling is a balance between the level of upstream activation of the pathway and the negative regulatory activity of Pten. In other studies, Pten loss has been shown to increase proliferation of neural stem cells in vitro, and during other developmental stages in the SVZ (Groszer et al. 2001; Gregorian et al. 2009). The proliferation consequences downstream of Pten loss are likely influenced by the extent of upstream activation of PI3K driven by the growth factor environment at different developmental stages.

In wild-type RMS, the PI3K-Akt-mTorc1 pathway is not activated in Dcx + cells until they reach the $\mathrm{OB}$, which correlates well with the time when they begin further differentiation. This suggests that the PI3K-Akt-mTorc1 pathway might be involved in the transition from a migratory neuroblast to a differentiating neuron in response to local environmental cues. In the absence of Pten, premature activation of PI3K signaling in neuroblasts in the RMS likely triggers precocious differentiation resulting in ectopic 
positioning. Inhibition of mTorc1 activity effectively rescued ectopic differentiation of neuroblasts and prevented the expansion of the SVZ and RMS, further supporting premature differentiation as a primary cause instead of a defect in the intrinsic migratory machinery. mTorc 1 modulates protein synthesis and cell growth and plays an important role in neuronal differentiation processes (Campbell and Holt 2001; Jaworski et al. 2005; Kumar et al. 2005; Tavazoie et al. 2005; Kwon et al. 2006; Wildonger et al. 2008; Chow et al. 2009). The rapamycin-mediated rescue of ectopic neurons in Pten ${ }^{c K O}$ brains occurred in the absence of proliferation effects. This could be explained by the recent finding that rapamycin potently inhibits S6K activity, but inefficiently and transiently inhibits 4EBP1 phosphorylation (Choo et al. 2008; Dowling et al. 2010). 4EBP1, independent of S6K, controls mTorc1-mediated cell proliferation (Dowling et al. 2010). Taken together, our results along with previous studies, suggest that proper temporal and spatial regulation of the PI3K-Akt-mTorc1 pathway plays an important role in regulating the appropriate timing and positioning for neuronal differentiation, but is not required for directional tangential neuronal migration. 


\title{
CHAPTER 3. CO-DELETION OF PTEN AND TRP53 SYNERGIZES IN GENERATING MEDULLOBLASTOMA WITH A GROWTH PATTERN CENTERING AROUND BLOOD VESSELS
}

\begin{abstract}
Introduction
Cells in the cerebellum arise from two germinal zones: the ventricular zone and the EGL. The ventricular zone is the germinal neuroepithelium during embryogenesis and generates deep cerebellar nuclei and Purkinje cells as well as other GABAergic neurons in the cerebellum (Goldowitz and Hamre 1998). Ptfla, a bHLH transcription factor, is required for the GABAergic neuron specification in the ventricular zone and loss of function resulted in complete loss of cerebellar GABAergic neurons (Sellick et al. 2004; Hoshino et al. 2005).
\end{abstract}

At birth, the ventricular germinal neuroepithelium disappears and proliferative precursors in the rhombic lip emigrate along the pial surface and form the EGL (Carletti and Rossi 2008). The EGL proliferates most during early postnatal days and produces all the internal granule cells (Wang and Zoghbi 2001). Multiple cell signaling pathways, for instance, cyclinD2 (Huard et al. 1999), p27 (Miyazawa et al. 2000), Shh (Dahmane and Ruiz i Altaba 1999), N-myc (Knoepfler et al. 2002), PI3Ks (Kenney et al. 2004) and Math1 (Ben-Arie et al. 1997) are crucial for cerebellar granule cell development among which the Shh signaling pathway is one of the best characterized. Shh, expressed by Purkinje cells, binds to its receptor, Ptch1, on granule cell precursors in the EGL, promotes proliferation and prevents their differentiation (Wallace 1999; Wechsler-Reya and Scott 1999). Unrestricted Shh signaling caused by Ptch1 mutation leads to medulloblastoma in both human and mouse (Goodrich et al. 1997). Pten is also required for the normal development of the cerebellum. Deletion of Pten in the granule progenitors in the EGL lead to ectopic positioning and hypertrophy of cerebellar granule cells (Backman et al. 2001; Kwon et al. 2001; Marino et al. 2002).

Aside from the two well characterized germinal zones in the developing cerebellum, multipotent neural stem cells have been isolated from the cerebellum postnatally and are thought to be located in the white matter tracts (Lee et al. 2005) which might arise from embryonic ventricular zone and emigrate into the white matter tracts (Zhang and Goldman 1996). The identity and function of this pool of neural stem/progenitor cells are largely unknown.

Medulloblastoma is a collection of clinically and molecularly heterogeneous diseases and can be divided into several subtypes (Thompson et al. 2006; Kool et al. 2008; Northcott et al. 2010; Cho et al. 2011). The subtype of medulloblastoma harboring mutations in Shh pathway including Ptch1 and Sufu is thought to originate from the EGL precursors and is supported by Ptch1 knock-out mouse models (Schuller et al. 2008; Yang et al. 2008). The Wnt subtype of medulloblastoma is likely to develop from cells of the dorsal brainstem outside of cerebellum (Gibson et al. 2010). Cells of origin for other medulloblastoma subtypes are still unknown. 
Cancer initiating cells, or cancer stem cells, are the subpopulation in the primary tumor that are capable of self-renewing, differentiating and regenerating its original tumor when transplanted in vivo. Cancer initiating cells have now been isolated from human cancers of the blood, breast, brain, skin, bone, and prostate (Ward and Dirks 2007). They are hypothesized to sustain tumor growth and be responsible for the relapse and metastasis. Cancer initiating cells could also be isolated from human medulloblastomas (Singh et al. 2003; Singh et al. 2004) and were shown to reside in a perivascular niche (Calabrese et al. 2007). The perivascular cell population were found to account for the re-growth of Ptch1-mutant medulloblastoma tumor after irradiationinduced apoptosis (Hambardzumyan et al. 2008). However, it is unknown whether there is a perivascular population of cells in the wild-type brain which is susceptible to oncogenic transformation and whether they could serve as cells of origin for medulloblastoma.

In addition to Shh and Wnt pathways, PI3K is another pathway aberrantly regulated in human medulloblastoma. Human medulloblastomas are found to overexpress RTKs such as ERBB2 (Hernan et al. 2003) and PDGFRB (MacDonald et al. 2001; Gilbertson and Clifford 2003). Proliferation of human medulloblastoma cells are dependent on activation of PI3K/AKT signaling perhaps caused by reduction of PTEN expression (Hartmann et al. 2006). Recent whole genome sequencing of 88 human medulloblastomas found PTEN mutations occur together with either PTCH1 or TP53 mutations (Parsons et al. 2011) which suggests a cooperative effect between PI3K and Ptch1 pathways. Mouse studies have also shown the synergistic effects of Ptch1 loss with Igf2, PI3K or Akt in generating medulloblastomas (Hahn et al. 2000; Rao et al. 2004; Riobo et al. 2006). However, the exact cooperative effects need further investigation.

In the present study, we inactivated Pten in the neural stem/progenitor cells at birth using a Nestin (a marker for the neural stem/progenitor cells) promoter driven CreER transgenic mouse and revealed a novel perivascular proliferative niche in the cerebellum. Co-deletion of Pten and Trp53 synergized in generating medulloblastoma with a growth pattern centering around blood vessels and extensive neuronal differentiation suggesting a potential new cell of origin for medulloblastoma.

\section{Experimental Procedures}

\section{Mice}

For Cre activity mapping, Nestin-CreER ${ }^{T 2}$ transgenic mice were bred with $R 26 R$ EYFP (Srinivas et al. 2001) mice to generate Nestin-CreER ${ }^{T 2} ; R 26 R-E Y F P$ mice. Cre activities were detected by EYFP IF staining on frozen tissue sections. Nestin$\mathrm{CreER}^{T 2}$ Pten $^{\text {loxP/loxP }}\left(\right.$ Pten $\left.^{\text {cKO }}\right)$ mice were generated by crossing Nestin-CreER ${ }^{T 2}$ mice to Pten ${ }^{\text {loxP }}$ mice (Suzuki et al. 2001), a gift from Tak Mak (University of Toronto, Toronto). Nestin-CreER ${ }^{T 2} ;$ Pten $^{\text {loxP/loxP }} ; \operatorname{Trp} 3^{\text {loxP/loxP }}\left(\right.$ Pten $\left.^{\text {cKO }} ; \operatorname{Trp}^{2} 3^{\text {cKO }}\right)$ mice were generated by 
breeding Nestin-CreER $R^{T 2} ;$ Pten $^{\text {loxPloxP }}$ with $\operatorname{Trp} 53^{\text {loxP }}$ mice (Jonkers et al. 2001). For all analyses including Nestin-CreER ${ }^{T 2}$, the transgene was hemizygous to avoid possible variation from transgene dosage. All procedures were reviewed and approved by the Animal Care and Use Committee at St. Jude Children's Research Hospital.

\section{Genotyping polymerase chain reaction assays}

PCR was used to genotype mice. Primers used were: $\mathrm{Cre}$, forward primer: 5'AGCGATCGCTGCCAGGAT; reverse primer: 5'ACCAGCGTTTTCGTTCTGCC; expected product: 157 base pairs. Pten, forward primer: 5'TTATCTGGATCAACTTTGGGCC; reverse primer: 5'TCCCACCAATGAACAAACAGT; expected products: 146 (wild-type) and 244 (loxP) base pairs. $\operatorname{Trp} 53$, forward primer: 5'CACAAAAACAGGTTAAACCCAG; reverse primer: 5'AGCACATAGGAGGCAGAGAC; expected products: 288 (wild-type) and 370 (loxP) base pairs.

\section{Induction of Cre activity}

TM (Sigma) was dissolved at $5 \mathrm{mg} / \mathrm{ml}$ at $37^{\circ} \mathrm{C}$ in corn oil (Sigma) and sterilized by filtering and stored at $4^{\circ} \mathrm{C}$ in the dark for up to 10 days. Pups were injected daily with $3 \mathrm{mg} / 40 \mathrm{~g}$ TM i.p. from P0-1. An equivalent volume of sterile filtered corn oil alone was used for vehicle injections. Two injections in the same mouse were separated by 24 hours.

\section{Immunohistochemistry and immunofluorescence}

Mice were anesthetized and perfused transcardially with 1x PBS followed by $4 \%$ PFA in PBS. Following dissection, tissues were post-fixed overnight in 4\% PFA in PBS at $4^{\circ} \mathrm{C}$, and then equilibrated in $25 \%$ sucrose in PBS for an additional 24 hours at $4^{\circ} \mathrm{C}$. Tissues were embedded in TBS embedding media (Triangle Biomedical Sciences) on dry ice and cut into $12 \mu \mathrm{m}$ thick cryosections. Tissue slides were equilibrated at room temperature for 20 minutes then washed three times in PBS prior to staining. For paraffin sections, mice were processed the same way as above. Following perfusion, tissues were post-fixed for an additional 24 hours in 4\% PFA, embedded in paraffin, and cut into $5 \mu \mathrm{m}$ sections. Primary antibodies used for immunostaining were against GFP (1:1000, Invitrogen A6455; 1:2000, Abcam \#13970), Pten (1:100, Cell Signaling \#9559), p-Akt S473 (1:50, Cell Signaling \#9271), Gfap (1:200, Sigma G3893), Ki67 (1:5000, Novocastra NCL-Ki67p), CD34 (1:100, BD Pharmingen \#553731), CD45 (1:400, BD Pharmingen \#553076), CD133 (1:100, eBioscience \#12-1331) and Nestin (1:100, Chemicon MAB353). For IHC, microwave antigen retrieval was performed for all antibodies and biotinylated secondary antibodies were used in conjunction with horseradish peroxidase-conjugated streptavidin (Elite ABC, Vector Laboratories). Color development was conducted with substrates NovaRed, DAB or VIP (Vector 
Laboratories) and counterstaining with hematoxylin or methyl green (Vector Laboratories), respectively. For IF, Alexa Fluor 488, 647 (Invitrogen) and cyanine 3, 5 (Jackson ImmunoResearch) conjugated secondary antibodies were employed along with Vectashield mounting media containing DAPI (Vector Laboratories).

\section{Array comparative genomic hybridization analysis}

Genomic DNA was prepared by scraping tumor tissues from multiple deparaffinized sections, followed by Proteinase K-digestion and phenol-chloroform extraction. DNA quality was verified by gel electrophoresis. Control DNA was isolated from the spleens and livers of wild-type FVB mice. A total of 20 Pten; p53 double cKO tumors were analyzed using the Roswell Park Cancer Institute (RPCI) 6.5K mouse BAC array comparative genomic hybridization $(\mathrm{aCGH})$.

\section{Fluorescent in situ hybridization analysis}

Fluorescent in situ hybridization (FISH) analysis was performed on paraffin sections by the St. Jude Cancer Center Core Cytogenetic Laboratory. Purified DNA from a BAC clone (RP24-70J8 cl.1) containing the murine Ptchl locus was labeled with digoxigenin-11-dUTP (Roche Molecular Biochemicals) by nick translation. The murine Ptch1 clone is known to localize to mouse chromosome band 13B3 so a biotinylated mouse 13 control clone (RP24-386H1O) was used as an internal control for the FISH assays. The Ptch 1 clone and the chromosome 13 control clone were combined with sheared mouse DNA and hybridized in a solution containing 50\% formamide, $10 \%$ dextran sulfate, and 2X SSC to the deparaffinized and pepsin treated slides. Specific hybridization signals were detected by incubating the hybridized slides in fluorescein labeled anti-digoxigenin (Roche Molecular Biochemicals) and Texas red avidin (Vector Laboratories Inc). The cells were then stained with DAPI and analyzed. A total of 200 interphase cells were scored from each sample for the number of green (70J8) and red signals (386HI0) per cell.

\section{Quantitative real-time polymerase chain reaction assay}

The same genomic DNA extracted for aCGH assay was used for real-time PCR analysis. The primer/probe set spanning Ptchl exon 2 was used, forward primer Ptch1ex2-SDS-F (5'-GGCTACTGGCCGGAAAGC), reverse primer Ptch1ex2-SDS-R (5'-GAATGTAACAACCCAGTTTAAATAAGAGTCT) and Taqman probe Ptch1ex2SDS-F (5'-CCGCTGTGGCTGAGAGCGAAGTTTC). Gapdh (located on mouse chromosome 6) was used as internal standards to normalize the data. Four nanograms of DNA from tumor samples or Gapdh DNA were amplified in TaqMan Fast Universal PCR Master Mix (Applied Biosystems) using a 7900HT Fast Real-Time PCR system (Applied Biosystems) $\left(95^{\circ} \mathrm{C}\right.$ for 20 seconds, then 40 cycles of $95^{\circ} \mathrm{C}$ for 5 seconds and $60^{\circ} \mathrm{C}$ for 30 seconds). Standard curve was generated from three-fold serial dilutions of 
Gapdh DNA. Quantitative real-time PCR for each sample was performed in triplicate and means were reported. The result of real-time PCR was analyzed with SDS ver2.3 software (Applied Biosystems).

\section{Ptch1 sequence analysis}

Ptch1 sequencing analysis was done as describe by Lee et al. (2006b). Total RNA was extracted from snap-frozen medulloblastoma samples using Trizol (Invitrogen). cDNA was synthesized from tumor RNA by reverse transcription using random hexamer primers and SuperScript II Reverse Transcriptase (Invitrogen). After cDNA synthesis, two rounds of PCR amplification were performed to obtain sufficient Ptch1 products for sequencing. The first round of PCR was done with forward primer Ptch1-14 (5'ACGCGCAATGTGGCAATGGAAGGC) and reverse primer Ptch1-R1 (5'GAAGCGGCCGCTTCAGATTTTAATTACCC) to amplify a full-length Ptch1.

Subsequently, the PCR products were further amplified with following five different sets of primers whose products overlapped each other and spanned a full-length of Ptch1. Set A: forward primer Ptch1-F (5'-ATGGCCTCGGCTGGTAACG) and reverse primer Ptch1-1 (5'-AAGGCCGGTCCATGTACCCATGGC); set B: forward primer Ptch1-2 (5'GCTTAATCATTACACCTTTGGACTGC) and reverse primer Ptch1-6 (5'AAAGGAGCATAGTGCTTCTCTGC); set C: forward primer Ptch1-5 (5'TTGAGCCACAGGCCTACACAGAGC) and reverse primer Ptch1-8 (5'GTCTGAGGTGTCTCGTAGGCCG), set D; forward primer Ptch1-7 (5' TGGGAAACTGGGAGGATCATGC) and reverse primer Ptch1-10 (5' GCTCAGGCGAAGGAGTGGGCAGTCG); and set E: forward primer Ptch1-9 (5'GTGGAGTTCACCGTCCACGTGGC) and reverse primer Ptch1-R2 (5'GAAGCGGCCGCTCAGTTGGAGCTGCTCCCCCACGGC) . The PCR products were sequenced by a routine Big Dye Terminator (v.3) Chemistry approach on Applied Biosystem 3700 DNA analyzer.

\section{Results}

\section{Nestin-CreER ${ }^{T 2}$ targets cerebellum at birth}

Nestin-CreER ${ }^{T 2} ; R 26 R-L a c Z$ or Nestin-CreER ${ }^{T 2} ; R 26 R-E Y F P$ mice were injected with TM to map the Cre activity at birth when brain is still undergoing active development. Consistent with the fact that developing brain has stronger expression of Nestin (Lendahl et al. 1990) and more common neural stem/progenitors, Cre activities in Nestin-CreER ${ }^{T 2}$ lines were more robust in immature brain with strongest activities in the neural stem/progenitor cell niches including the SVZ and the EGL of cerebellum (Figure 2-2C). The bulk of cerebellum develops postnatally, so Nestin-CreER ${ }^{T 2}$ could target the cerebellum including the EGL, IGL and white matter tracts very effectively with TM induction at birth (Figure 3-1). Littermate control with vehicle injection showed very low level of TM-independent leaky Cre activity (Figure 3-1). 
Vehicle

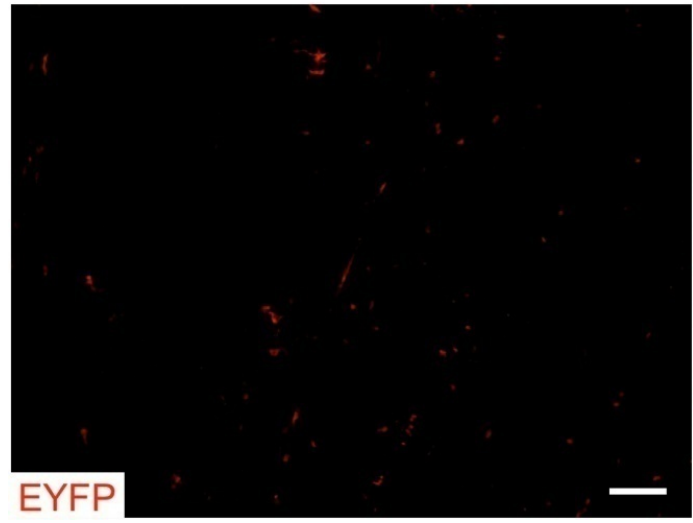

TM

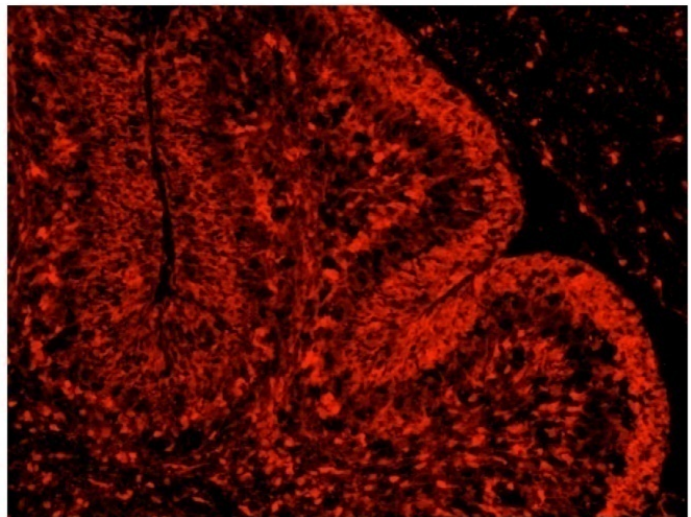

Figure 3-1. Nestin-CreER $R^{T 2}$ targets cerebellum at birth.

EYFP IF staining of the cerebellum from mice induced with vehicle or TM from P0-1. Tissue was harvested at P5. Strong EYFP signals were noticed in the EGL, IGL and white matter tract of cerebellum in the TM-induced mice whereas mice with vehicle induction had only very low level of EYFP. Scale bar: $100 \mu \mathrm{m}$. 


\section{Deletion of Pten at birth resulted in disorganization and hypertrophy of cerebellum which mimics human Lhermitte-Duclos disease}

We induced the deletion of Pten in the Nestin-CreER ${ }^{T 2}$ Pten ${ }^{\text {IoxPloxP }}\left(\right.$ Pten $\left.^{\text {cKO }}\right)$ neonate mice to see the effect of broad Pten loss on a developing brain. The Pten ${ }^{c K O}$ mice did not show obvious abnormalities until 2-3 months of age when the animals started to exhibit lethargy, progressive enlargement of the skull and progressive neurological abnormalities including ataxia and seizure. One hundred percent of the mice (16/16) induced at birth were affected whereas none of the un-induced Pten ${ }^{c K O}$ mice or TMtreated wild-type littermates showed these deficits. Animals had to be euthanized due to severe ataxia and brain hypertrophy with a median survival of 153 days (Figure 3-2C). In addition to the dramatic expansion of the SVZ and RMS described in Chapter 2, brains from $P t e n^{c K O}$ mice induced with TM at birth showed profound hydrocephalus and dramatic increases in overall sizes especially in cerebellum (Figure 3-2A). The foliation was visible but the organization of cell layers was disrupted in the cerebellum. The molecular layer (ML) was dramatically thickened with many ectopic granule cells deficient of Pten staining. The majority of Purkinje cells were positive for Pten staining but the normal monolayer structure was not nicely formed. The IGL was thinner than wild-type control and composed of both Pten positive and negative granule cells (Figure 3-2B). The size of the Pten-deficient granule cells is larger than the Pten positive granule cells in aging mice (not shown). All these abnormalities mimic human Lhermitte-Duclos disease and are in agreement with previous findings but in a more severe manifestation (Backman et al. 2001; Kwon et al. 2001).

\section{Pten inactivation at birth revealed novel ectopic perivascular proliferative niches in the cerebellum}

In addition to the cerebellar disorganization and hypertrophy, we identified multiple perivascular proliferative niches in the cerebellum (20/24 mice) when deleting Pten in neonatal neural stem/progenitor cells at birth, which have never been observed before in previous Pten ${ }^{c K O}$ mouse models (Backman et al. 2001; Kwon et al. 2001; Fraser et al. 2004). The perivascular hyperplastic lesions were observed as early as 3 months of age but were not found in 30-day-old mice (data not shown). These hyperplastic lesions grew surrounding CD34+ blood vessels and had high percentage of Ki67+ cells (Figure 3-3). The perivascular proliferative lesions varied in their sizes from as few as several cells to hundreds of cells. They were distributed throughout cerebellum but were more frequently found in anterior and lateral cerebellar lobules and in areas around the pia between lobules (data not shown). They are not infiltrating inflammatory cells (CD45-) but have a progenitor-like appearance. They tend to express Nestin and CD133 which mark neural stem/progenitor cells or brain tumor initiating cells (Lendahl et al. 1990; Singh et al. 2003; Singh et al. 2004; Coskun et al. 2008) as well as Gfap that labels neural stem cells in the SVZ (Doetsch et al. 1999)(Figure 3-3). These perivascular precursor-like cells are Pten null and p-Akt positive indicating that they derived from cells that underwent Cre-mediated recombination (Figure 3-3). However, these perivascular 

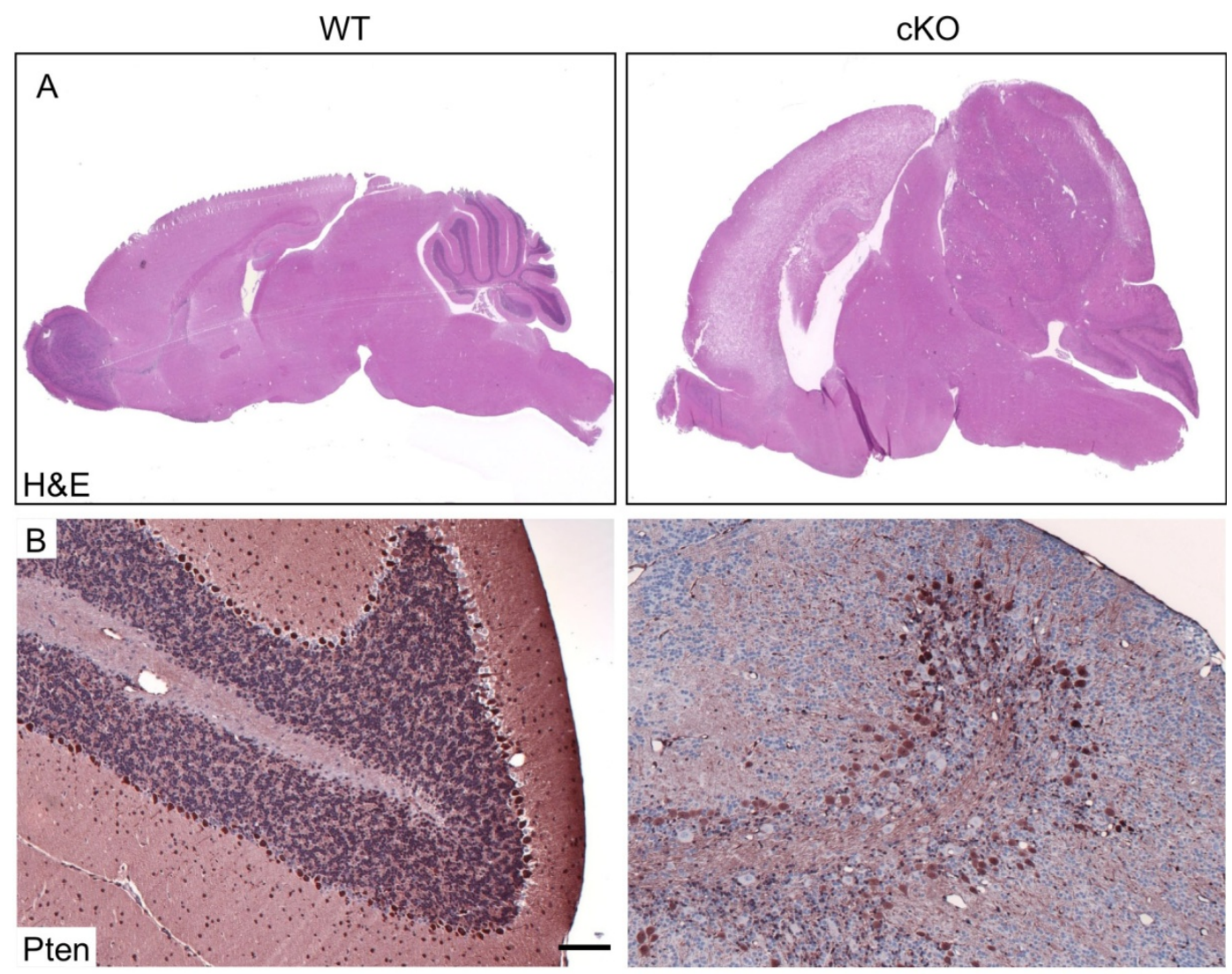

C Survival of $P \operatorname{Pten}^{c K O}$ :Survival proportions

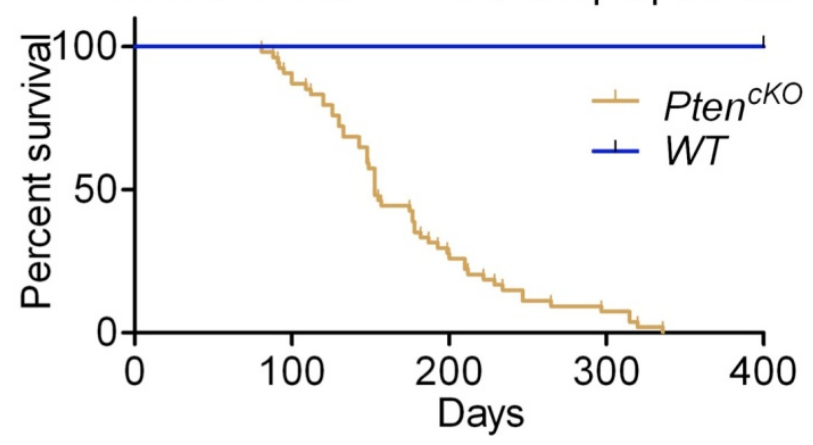

Figure 3-2. Deletion of Pten at birth resulted in disorganization and hypertrophy of cerebellum.

Representitive H\&E (A) and Pten (B) IHC staining of the cerebella from mice induced with TM at birth. (A) H\&E staining of the wild-type (WT) and Pten ${ }^{c K O}$ (cKO) brains showed dramatic hypertrophy of the Pten ${ }^{c K O}$ brain. (B) Pten IHC staining of the WT and cKO brains showed disrupted organization of the cerebellum and ectopic Pten-deficient granule cells in the molecular layer in the Pten ${ }^{c K O}$ brain. Scale bar: $200 \mu \mathrm{m}$. (C) KaplanMeier survival curves shows $\mathrm{Pten}^{c K O}$ mice had a median morbidity age of 153 days due to macrocephaly and hydrocephaly (brown curve). 


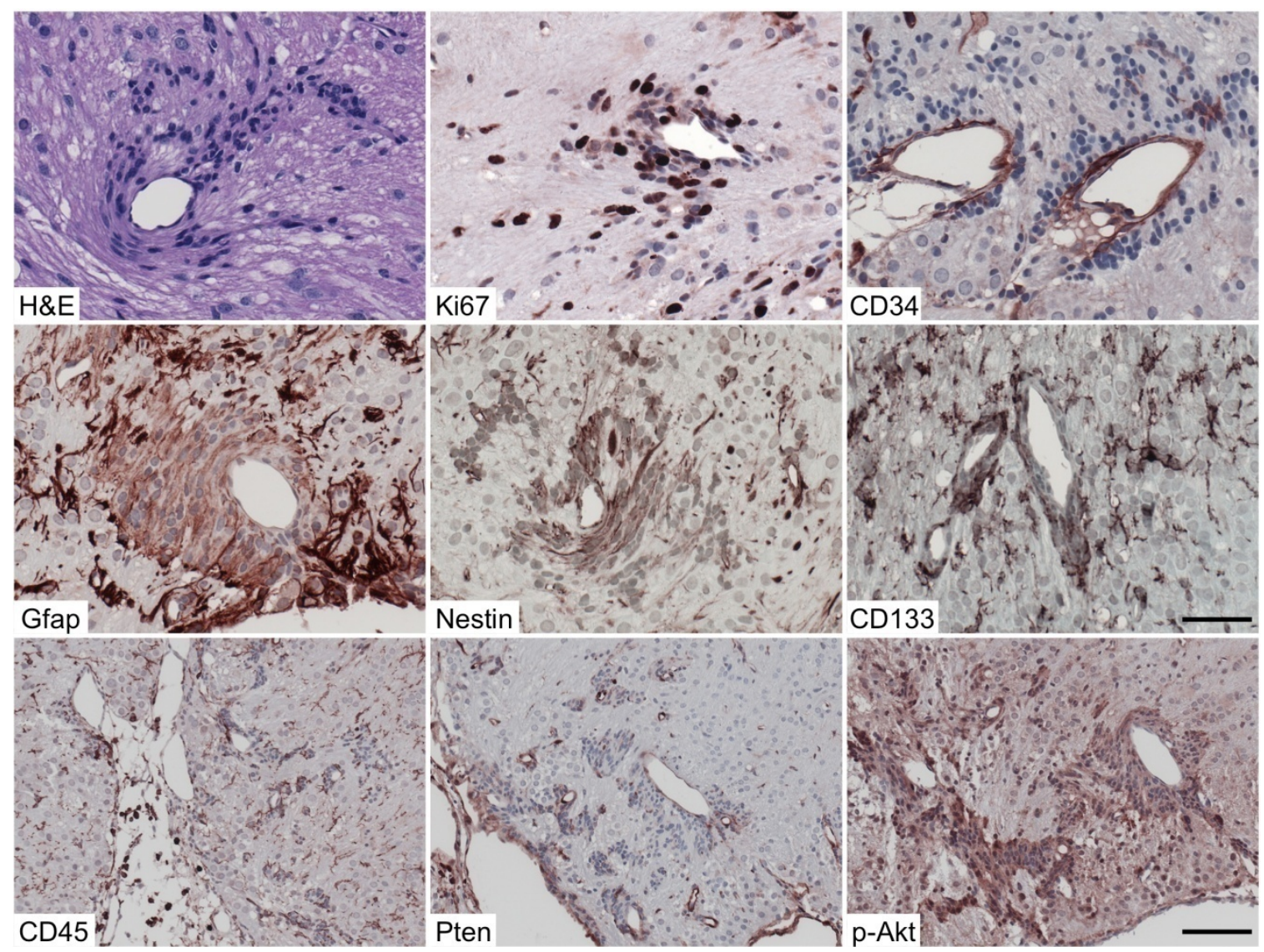

Figure 3-3. Pten inactivation at birth reveals novel perivascular proliferative niches in the cerebellum.

IHC staining as indicated of the cerebella from mice induced with TM at birth. The perivascular lesion growing surrounding CD34+ blood vessels has a high percentage of Ki67+ cells. The perivascular cells are negative for CD45, a marker for leukocytes, but have progenitor-like appearance. They tend to express Nestin, CD133 and Gfap indicating their neural stem/progenitor identity. These perivascular precursor-like cells are Pten null and positive for p-Akt indicating they are derived from cells that underwent re-mediated recombination. Scale bar: CD45, Pten and p-Akt $=100 \mu \mathrm{m}$, others $=50 \mu \mathrm{m}$. 
hyperplastic lesions were never found to develop into full-blown tumors in mice up to 10 months of age.

\section{Co-deletion of Pten and Trp53 at birth resulted in medulloblastoma with unusual vascularization}

Since TP53 is one of the most frequent mutations in several common primary human brain tumors (Frank et al. 2004; Parsons et al. 2008; TCGA 2008; Parsons et al. 2011), we asked whether or not loss of Pten during early postnatal development would cooperate with Trp53 deficiency in generating full-blown cerebellar malignancy. NestinCreER $R^{T 2}$;Pten ${ }^{\text {loxPloxP }}$ mice were bred to Trp $53^{\text {loxP }}$ mice to generate Nestin-

$\mathrm{CreER}^{\text {T2 }} ;$ Pten $^{\text {loxP/loxP }} ; \operatorname{Trp} 53^{\text {loxP/loxP }}(\mathrm{dKO})$ mice and induced at birth. All of the dKO mice (26/26) developed cerebellar tumors which resemble human medulloblastoma with a median tumor onset of 63 days of age and Nestin-CreER ${ }^{\text {T2 }} ;$ Pten $^{\text {loxP/+ }} ;$ Trp $53^{\text {loxPloxP }}$ mice $(22 / 25)$ succumbed to the same tumor with a one-month delay in onset compared to dKO mice (Figure 3-4D and data not shown). All the dKO medulloblastomas manifested as highly cellular neoplasms of classic medulloblastoma pathology composed of small blue cells with two striking features, high vascularization and perivascular clustering of tumor cells, which distinguish this tumor model from other existing mouse medulloblastoma models (Figure 3-4A and B). Pten IHC staining showed that the vast majority of the tumor bulk was devoid of Pten with only endothelial cells retaining Pten (Figure 3-4C). The majority of the Nestin-CreER $R^{T 2}$; Trp5 $3^{\text {loxPloxP }}$ mice have to be euthanized for peripheral tumors probably due to leaky Cre activity outside of CNS (Figure 3-4D). Interestingly, the Nestin-CreER $R^{T 2} ;$ Pten $^{\text {loxPloxP }} ; \operatorname{Tr} p 53^{\text {loxP/+ }}$ mice (5/8) developed glioma like malignancy in the cerebellum instead of medulloblastoma by 5-6 months old (see Appendix). Besides cerebellum, the $\mathrm{dKO}$ and Nestin-CreER ${ }^{T 2} ;$ Pten $^{\text {loxP/loxP }} ; \operatorname{Trp} 53^{\text {loxP/+ }}$ mice also developed glioma like tumors in the forebrain (18/26, 5/8 respectively) and brain stem $(5 / 26,8 / 8)$ (see Appendix).

\section{Pten; Trp53 double knock-out medulloblastoma showed a growth pattern centering around blood vessels}

The dKO medulloblastomas were highly proliferative and showed predominant neuronal differentiation by IHC staining which confirms their identity as primitive neuroectodermal tumors (Figure 3-5). Most interestingly, these highly vascularized medulloblastomas often exhibited a growth pattern centering around blood vessels. The tumor cells showed a mature neuronal immunophenotype except those around blood vessels which expressed primitive markers including Nestin (Figure 3-5). At the edges of the tumors that were invading the non-tumor brain parenchyma, the Nestin+ and Ki67+ proliferative cells were often located immediately around blood vessels, which are reminiscent of the perivascular proliferative niches in the Pten $^{c K O}$ cerebellum (Figure 3-3 and Figure 3-5). These data suggest that the dKO medulloblastomas might initiate from the perivascular proliferative niches in the $\mathrm{Pten}^{c K O}$ cerebellum. 

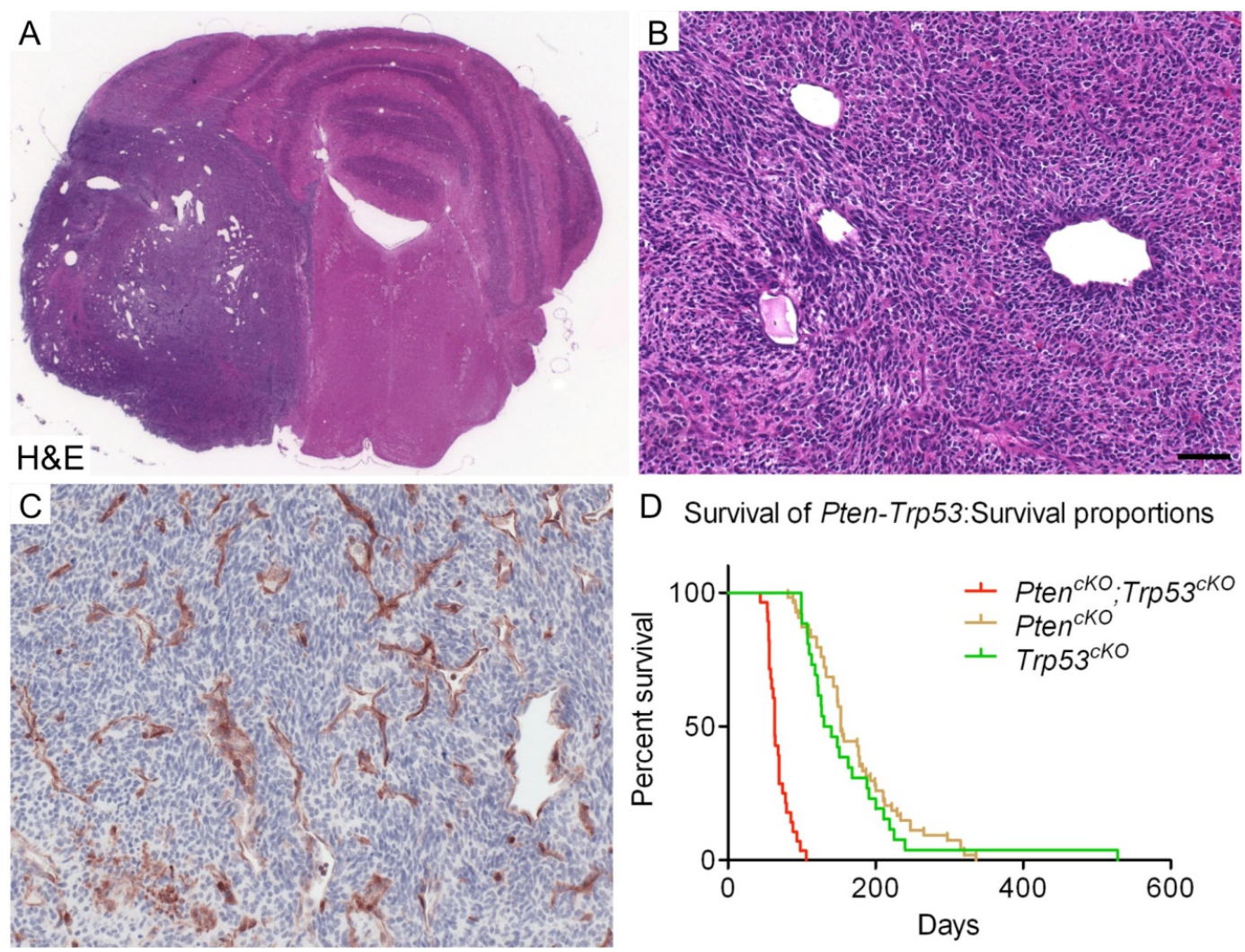

D Survival of Pten-Trp53:Survival proportions

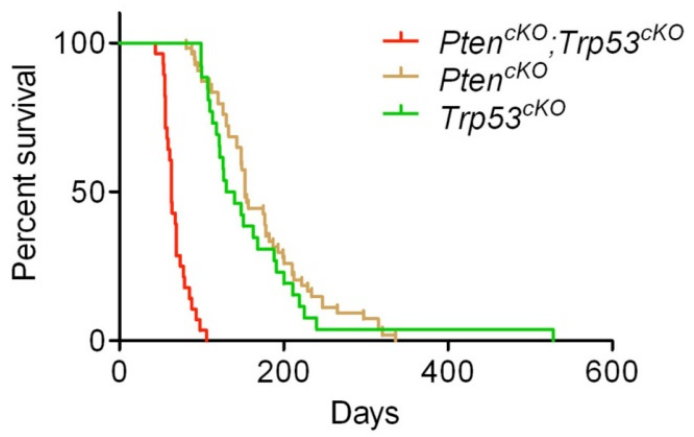

Figure 3-4. Co-deletion of Pten and Trp53 at birth resulted in medulloblastoma with unusual vascularization.

(A and B) Representative H\&E staining of medulloblastoma generated by co-deletion of Pten and Trp53 at birth shows pathology of classic medulloblastoma and high vascularization. (C) Pten IHC staining shows $\operatorname{Pten}^{c K O} ; \operatorname{Trp}^{2} 3^{c K O} \mathrm{dKO}$ medulloblastoma cells are devoid of Pten except for the blood vessel. (D) Kaplan-Meier survival curves show Pten ${ }^{c K O} ; \operatorname{Trp}_{53} 3^{c K O}$ dKO mice with a median tumor onset of 63 days of age (red curve), Pten $^{\text {cKO }}$ mice with a median morbidity age of 153 days of age (brown curve) and NestinCreER ${ }^{T 2} ; \operatorname{Trp} 53^{\text {loxP/loxP }}\left(\operatorname{Trp} 53^{\text {cKO }}\right.$ )with a median morbidity age of 135 days (green curve). Pten $^{c K O}$ mice were euthanized due to macrocephaly and hydrocephaly. The majority of $\operatorname{Trp} 53^{c K O}$ were euthanized because of peripheral tumors. All genotypes were induced with TM at birth. Scale bar: B and $C=50 \mu \mathrm{m}$. 

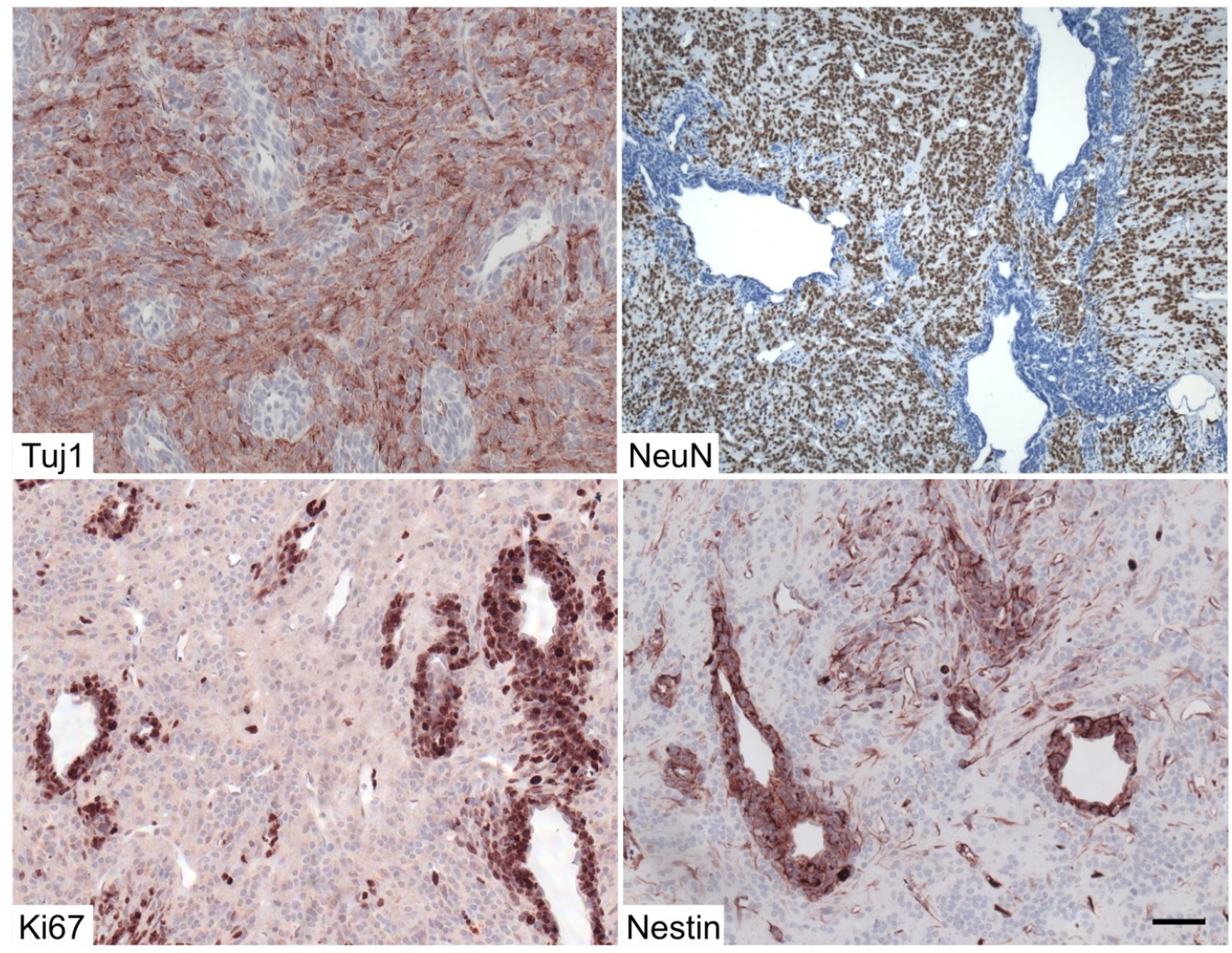

Figure 3-5. Pten;Trp53 double knockout medulloblastoma showed a growth pattern centering around blood vessels.

IHC staining as indicated of the medulloblastomas from $\mathrm{dKO}$ mice induced with $\mathrm{TM}$ at birth. Tuj1+ and NeuN+ IHC show the dKO medulloblastomas exhibited a neuronal immunophenotype except those cells around blood vessels. Nestin+ and Ki67+ IHC staining of the edges of the medulloblastomas show proliferative pregenitor cells were often located immediately around blood vessels that were invading the non-tumor brain parenchyma. Scale bar: $50 \mu \mathrm{m}$. 


\section{Pten;Trp53 double knock-out medulloblastoma spontaneously lost chromosome 7, 13 and 16}

Tumorigenesis is considered a multi-step process involving initiation, promotion and progression. To understand what other secondary genetic alterations in addition to the initiating Pten and Trp53 mutations might be involved in the medulloblastoma formation, we did aCGH comparing genomic DNA from the tumors and reference genomic DNA from liver and spleen. We identified 3 most recurrent and consistent genomic abnormalities in tumor samples. All of the 18 medulloblastomas showed loss of chromosome 13 on which Ptch1 is located (Figure 3-6). In addition, 5 of the tumors had focal homozygous deletion of the BAC probe encompassing Ptchl. Thirteen and eighteen out of the 18 medulloblastomas also showed loss of the entire or part of chromosome 7 and 16 respectively (Figure 3-6). No recurrent focal homozygous deletion on chromosome 7 or 16 could be found. Interestingly, two of the cerebellar nonmedulloblastoma tumors included in this aCGH analysis displayed a completely different landscape of genomic alterations than the medulloblastoma samples and failed to show loss of chromosomes 7 or 13 (Figure 3-6).

\section{Spontaneous deletion and mutation of Ptch1 occurred in Pten;Trp53 double knock- out medulloblastomas}

To further investigate whether Ptchl was the target gene of the genomic alteration on chromosome 13, we performed FISH analysis and detected loss of at least one allele of Ptchl in two medulloblastoma samples used for aCGH analysis (Figure 3-7A). Realtime PCR assay on the medulloblastoma genomic DNA further confirmed the copy number loss of Ptchl in both tumor samples (Figure 3-7B). We also performed RT-PCR and direct sequencing to analyze Ptch1 mRNA and found mutations of Ptch1 in another two medulloblastomas samples used for aCGH analysis (Figure 3-7C). These data support the hypothesis that Ptchl is the tumor suppressor gene on chromosome 13 that was selectively inactivated in $\mathrm{dKO}$ medulloblastoma by loss of heterozygosity.

\section{Discussion}

In this study, we used an inducible Nestin promoter driven Cre and deleted Pten in a wide-spectrum of the progenitors in cerebellum at birth when the brain is still undergoing active development. We recapitulated the phenotyes of neuronal hypertrophy and ectopic positioning found in previous studies and confirmed that Pten regulates neuronal size and positioning (Backman et al. 2001; Kwon et al. 2001; Fraser et al. 2004). The more severe phenotypes of cerebellar disorganization and hypertrophy compared to previous studies might be because of the more potent and ubiquitous Cre activity in neural stem/progenitor cells driven by the Nestin promoter than by the Gfap promoter used previously. An unexpected finding in this study is that we revealed a novel perivascular proliferative progenitor niche in the cerebellum by deletion of Pten at birth which has never been found in any other studies. This suggests the presence of an 

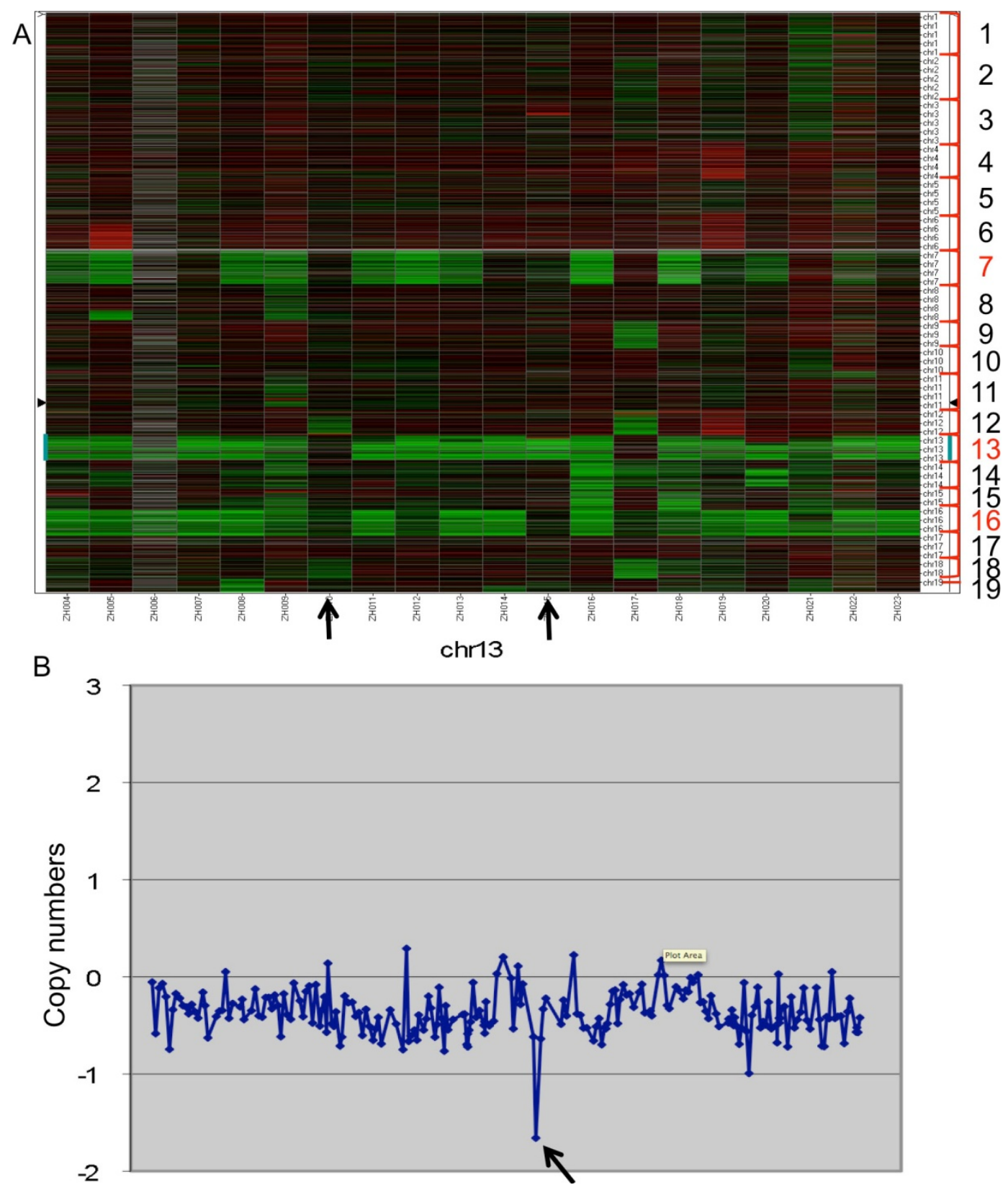

Figure 3-6. Pten; Trp53 double knockout medulloblastoma spontaneously lost chromosome 7,13 and 16.

(A) aCGH heat map of cerebellar tumors from Pten;Trp53 dKO mice induced with TM at birth. Each column represents one tumor sample. Numbers on the right indicate the chromosomes. The green color indicates copy number loss whereas red indicates copy number gain. All medulloblastoms (the two samples indicated by arrows were from nonmedulloblastoma samples) had loss of chromosome 7,13 and 16 as highlighted in red. (B) aCGH analysis of chromosome 13 from one representative medulloblastoma sample shows hemizygous deletion of whole chromosome 13 and homozygous deletion at one BAC probe containing Ptchl as indicated by an arrow. 


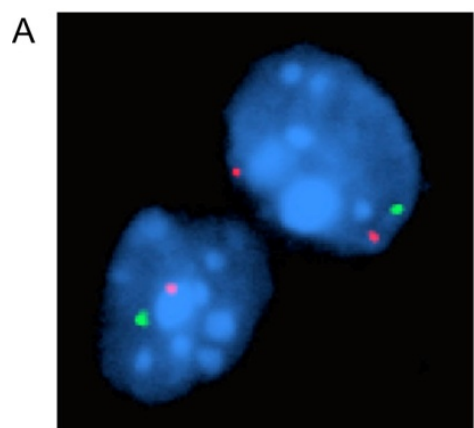

Red: Ch.13 ctrl

B

Green: Patched1

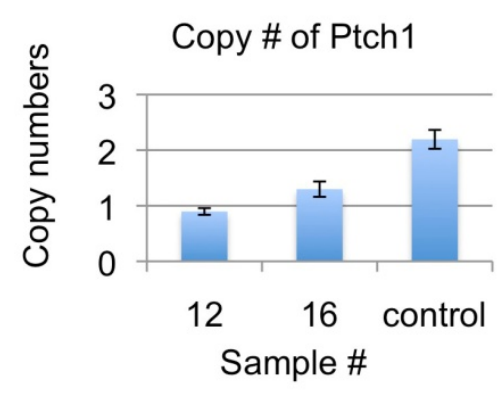

Ins GG
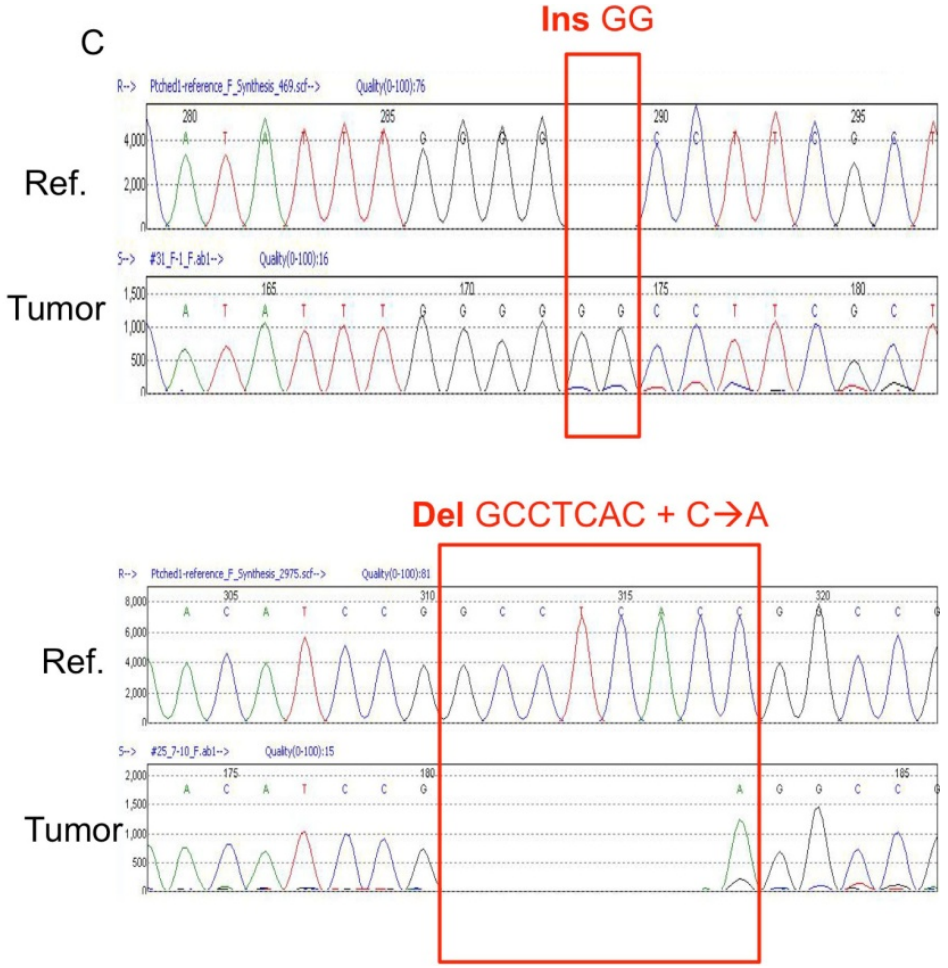

Figure 3-7. Spontaneous deletion and mutation of Ptch1 in Pten;Trp53 double knockout medulloblastoma.

(A) FISH analysis of a representative Pten;Trp53 dKO medulloblastoma sample. Red dot is a BAC probe located on one end of chromosome 13 outside of Ptch1 and green dot is a BAC probe containing Ptch1. (B) Quantitative real-time PCR analysis of Ptch1 on genomic DNA from dKO medulloblastomas. (C) Sequencing of cDNA synthesized from Ptch1 mRNA of 2 dKO medulloblastomas. Red boxes indicate the mutations by comparing reference wild-type sequence (Ref.) with medulloblastoma sequences (Tumor). 
unknown perivascular germinal niche in the developing cerebellum that is normally regulated by Pten but regresses upon completion of development, because we could not see the niche anymore when deleting Pten in adulthood (data not shown). Alternatively, Pten deletion may have perturbed normal development and resulted in a perivascular germinal niche that does not normally exist. Our study is consistent with the previous study that identified neural stem cells in the murine postnatal cerebellum (Lee et al. 2005) and suggests that the neural stem cells in the cerebellum are probably localized in a vascular niche. This is consistent with the finding that the SVZ neural stem cells also reside in a vascular niche (Mirzadeh et al. 2008; Shen et al. 2008; Tavazoie et al. 2008). We tried to grow neurospheres from both wild-type and Pten $^{\text {cKO }} \mathrm{P} 21$ cerebella but did not get consistent results (data not shown). Our study also underlines the importance of Pten regulation in the neural stem/progenitor cells in the cerebellum indicating Pten is required for silencing or withdrawing the neural stem cells upon completion of development under physiological conditions. Our results are consistent with the finding that Pten negatively regulate proliferation and self-renewal of embryonic neural stem cells (Groszer et al. 2001; Groszer et al. 2006) and is novel in that Pten loss caused spatially and temporally ectopic perivascular progenitor niches.

The cerebellar perivascular hyperplasia, however, did not show progressive expansion up to 10 months of age and was never found to be transformed into full-blown tumor masses. This is consistent with our finding in Chapter 2 that Pten deletion is not sufficient to initiate tumorigenesis in neural stem/progenitor cells in the fore-brain and is in contrast to Pten function in other stem cells. For instance, in hemopoeitic stem cells, inactivation of Pten causes short-term expansion, but long-term decline and eventual leukemomogenesis (Yilmaz et al. 2006; Zhang et al. 2006) indicating a tissue-specific stem cell regulation by Pten. Activation of oncogenic signals such as c-Myc, E1A and Ras could not only promote proliferation and growth, but also induce cell cycle arrest and cell death (Askew et al. 1991; Lowe and Ruley 1993; Serrano et al. 1997) constraining tumorigenesis. Pten deficiency, a strong oncogenic stress, has been shown to induce p53, $\mathrm{p} 16^{\text {Ink4a }}$ or $\mathrm{p} 19^{\text {Arf }}$ and cause cellular senescence in prostate and hematopoietic cells (Chen et al. 2005; Lee et al. 2011). This might account for the limited cerebellar perivascular hyperplasia observed in this study. So we deleted Trp53, another common mutated tumor suppressor in human brain tumors in addition to deletion of Pten, which indeed resulted in medulloblastoma. This corresponds to recent large-scale human medulloblastoma sequencing data showing that PTEN and TP53 mutations occured simultaneously (Parsons et al. 2011). Therefore inactivation of PTEN and TP53 might also synergize in generating human medulloblastoma.

The Pten and Trp53 dKO medulloblastoma in this study has very unique features that distinguish it from other mouse medulloblastoma models (Wetmore et al. 2000; Lee and McKinnon 2002; Zindy et al. 2003). Firstly, our medulloblastoma is highly vascularized manifesting as high-density of capillaries, extensive arborization and glomeruloid structures. This difference could be explained by the Pten inactivation in our medulloblastoma model and consequent PI3K pathway activation which have been shown to increase tumor angiogenesis through induction of VEGF or HIF1 in various tumor types (Giri and Ittmann 1999; Jiang et al. 2000; Zhong et al. 2000; Wen et al. 
2001). In supporting this idea, one recent study demonstrates that loss of one copy of Pten promoted tumorigenesis and increased angiogenesis in a mouse medulloblastoma driven by over-expression of SmoA1 (Castellino et al. 2010). In addition, the potential perivascular origin of intrinsically angiogenic cells for this medulloblastoma might also account for its extensive vascularization. Secondly, our medulloblastoma is special in that it grows exclusively around blood vessels and show strong neuronal differentiation of cells not adjacent to blood vessels. This illustrates a lineage hierarchy of cancer cells in which medulloblastoma cancer stem/progenitor cells maintain themselves in a perivascular niche and start to differentiate when migrating out from the niche. This is consistent with a previous study showing the perivascular Nestin-expressing tumor cells were responsible for the recurrence of Ptch1 and Pten dKO medulloblastoma after irradiation (Hambardzumyan et al. 2008). The extensive neuronal differentiation of our Pten null medulloblastoma in contrast to the medulloblastoma models with intact Pten which show less neuronal differentiation underscores the role of Pten inactivation and PI3K activation in promoting neuronal lineage differentiation of medulloblastoma cells. This is supported by two other medulloblastoma models with extensive nodularity of neuronal differentiation in which Pten is inactivated (Hambardzumyan et al. 2008; Castellino et al. 2010).

Human medulloblastoma is a heterogeneous disease that can be classified into several molecular subgroups based on genomic and expression profiles, one of which is enriched for mutations in sonic hedgehog (SHH) pathway (Thompson et al. 2006; Kool et al. 2008; Northcott et al. 2010; Cho et al. 2011). Mouse models of spontaneous medulloblastoma seem to over-represent the SHH subgroup of human disease in that the majority of mouse medulloblastomas harbor Ptch1 mutation (Wetmore et al. 2000; Zindy et al. 2007; Frappart et al. 2009) especially when involving Trp53 mutation. Our Pten and Trp53 dKO medulloblastoma also spontaneously developed Ptch1 mutations as well as other genomic abnormalities. One hundred percent penetrance of medulloblastoma and Ptch1 mutation indicates strong cooperation between PI3K, P53 and SHH pathways and potent selection pressure for Ptch1 inactivation in murine medulloblastoma genesis. Our tumor model is also very relevant to human disease given the recent sequencing data show PTEN mutation occurs exclusively together with either TP53 or PTCH1 mutations in all the medulloblastoma samples harboring PTEN mutation (Parsons et al. 2011). Although the subtype of SHH medulloblastomas are shown to be able to arise from the EGL (Schuller et al. 2008; Yang et al. 2008), our study suggests a potential new cell of origin for this subgroup. 


\title{
CHAPTER 4. GENERAL DISCUSSION AND FUTURE DIRECTIONS
}

\author{
Pten and Postnatal Brain Development
}

\section{Pten and postnatal neurogenesis}

Postnatal brain development involves neurogenesis and gliogenesis as well as other processes such as angiogenesis. Postnatal neurogenesis mainly happens in three brain regions: the SVZ-RMS-OB, cerebellum and dentate gyrus. The present study showed that loss of Pten cause ectopic neuronal differention in both the SVZ-RMS-OB and cerebellum suggesting a unified Pten function in neurogenesis in different compartments of brain. However, Pten loss also showed region-specific phenotypes: the expansion is more dramatic on ventral and dorsal parts of the SVZ but less severe in the middle part which might be explained by the heterogeneity of the neural stem cells in the SVZ (Merkle et al. 2007).

Neural stem cells in the SVZ reside in a niche with highly organized architecture in which they contact the ventricle with apical processes and blood vessels with basal processes (Mirzadeh et al. 2008; Shen et al. 2008; Tavazoie et al. 2008). In the expanded $\mathrm{Pten}^{c K O}$ SVZ, this specialized organization seems to be disrupted in that the proliferative progenitor cells are intermingled with differentiated neurons and sometimes located on the edge of the expansion away from the ventricle. Surprisingly, this altered architecture did not interfere with neurogenesis, given that no substantial changes in proliferation or apoptosis and no signs of an exhausted pool of neural stem cell were detected. In-depth analysis of the Pten ${ }^{c K O}$ SVZ by immunostaining or electron microscopy could provide specific insights into the relationship of neural stem cells to the ventricle and blood vessels in an altered niche.

The present study shows deletion of Pten in neuroblasts caused ectopic differentiation without disrupting directional migration. This suggests the migration defect of Pten null neuroblasts is secondary to a premature differentiation in which programmed differentiation was accelerated and neuroblasts lost their migration capability before they reached their normal destination. Future experiments need to be done looking for direct evidence of precocious differentiation, for instance, by comparing wild-type and Pten $^{c K O}$ neuroblasts at several timepoints after acute Pten deletion to see whether the differentiation program in Pten $^{c K O}$ cells is expedited by examining temporal differentiation markers. Our rapamycin treatment experiment showed that the premature differentiation of $\mathrm{Pten}^{c K O}$ is dependent on mTorc1. Future study needs to address whether terminal differentiation of wild-type neuroblasts is also dependent on mTorc 1 by knocking out mTorc 1 components. We could not address this by rapamycin treatment because rapamycin differentially inhibits downstream signaling of mTorcl (Choo et al. 2008; Dowling et al. 2010) and has inconsistent penetration and inhibition in different compartments of brain which often results in incomplete inhibition. However, one 
potential pitfall is that complete abolishion of mTorc1 activity might be incompatible with cell survival.

One interesting phenomenon is that ectopic differentiated Pten-null neurons were distributed throughout the SVZ-RMS-OB indicating that $\operatorname{Pten}^{c K O}$ neuroblasts terminated their migration at different places. The possible reasons for this could be firstly that neuroblasts are born and start their migration at different regions of the SVZ. Secondly, deletion of Pten may have a stochastic effect on the termination of neuroblast migration. So it is possible mTorc1 signaling is not activated yet in the Pten ${ }^{c K O}$ neuroblasts maintaining directional migration at the timepoint of observation because the activation of mTorc1 pathway is determined by Pten status as well as other unknown factors. For instance, engagement of ligands (external cues) with tyrosine kinase receptors on the membrane is likely needed in the Pten ${ }^{c K O}$ neuroblast for full activation of PI3K-AktmTorc1 pathway but might not be readily available to every neuroblast at a given timepoint. This might actually be a reflection of how neuroblasts terminate their migration naturally because in the wild-type brain migrating neuroblasts could stop anywhere throughout the whole OB layers. Future experiments could examine the status of mTorc 1 signaling in the Pen $^{c K O}$ neuroblasts that still maintain directional migration by means of, for instance, double labeling of EYFP and p-S6. Thirdly, migrating neuroblasts are a heterogeneous population (Merkle et al. 2007) that might respond to loss of Pten differentially. This is not likely the reason, however, because Pten null neurons were found to be in all of the OB layers that the neuroblasts normally integrate into. Since the neuroblasts give rise to multiple types of interneurons in the OB (Ihrie and AlvarezBuylla 2008), specific markers including TH, calbindin and calretinin for these interneurons could be used to examine if any of the interneuron subtype is more susceptible to Pten loss.

\section{Pten and postnatal gliogenesis}

Unlike neurogenesis which mainly happens during the embryonic stage, gliogenesis including astrocytogenesis and oligodendrocytogenesis peaks postnatally (Sauvageot and Stiles 2002). Loss of Pten resulted in hypertrophy and hyper-proliferation of astrocytes when using a Cre deleting Pten in both neurons and astrocytes during embryonic development (Fraser et al. 2004). This effect also contributed to the expansion of the SVZ and hypertrophy of the whole brain observed in this study. In the expanded $\mathrm{SVZ}$ of $\mathrm{Pten}^{c K O}$ but not wild-type mice, there were many hypertrophic astrocytes intermingled with retained Dcx + neuronal precursors and differentiated NeuN + mature neurons (see Appendix). There were also a huge number of hypertrophic astrocytes throughout the brain when Pten was deleted with the Nestin-CreER ${ }^{T 2}$ transgene at birth (data not shown). However, astrocyte hypertrophy or hyper-proliferation could not be detected when Pten was deleted in the developmentally mature brain (Chow et al. 2011). The discrepancy in these studies is likely because Pten was deleted at different developmental stages or in different types of cells indicating cell-type-specific or stagespecific Pten functions in astrocytes. Future study needs to find out whether the hypertrophic astrocytes in the Pten $^{c K O} \mathrm{SVZ}$ are differentiated from Pten null neural 
stem/precursor cells or whether they are reactive astrocytes due to a non-cell-autonomous effect of Pten loss. It is not clear if there is a specific cell context in which loss of Pten will cause a cell-autonomous hypertrophy and hyper-proliferation of astrocytes. Preliminary data show that there were far fewer hypertrophic astrocytes and very few EYFP+ astrocytes in the SVZ when Pten was deleted by Cre electroporation compared to by the inducible Nestin-CreER $R^{T 2}$ transgene. This suggests the astrocytosis in the Pten ${ }^{c K O}$ SVZ is likely a non-cell-autonomous effect (data not shown).

\section{Remaining questions on Pten and development}

Other questions raised in this study are what is the relationship between cell migration and differentiation? Are these two processes inter-linked or independent? Our study suggests that migration and differentiation are coordinated with each other. However, to what extent are they inter-connected? Does interruption of one process necessarily affect the other process? How is the differentiation affected in the neuroblasts in which only the migration machinery, for instance, mutations in PAR6 $\alpha$, LIS1, NUDEL, CDK5 and DCX, is interrupted.

Why is there a discrepancy regarding whether Pten is required for directional migration in both Dictyostelium and mammalian cells? Is the discrepancy due to a cellcontext dependent effect of Pten on directional migration or simply technical issues?

What are the cues that tell the migrating neuroblasts to stop and differentiate in the OB? One of the candidates is reelin, a critical extracellular protein regulating neuronal positioning (D'Arcangelo et al. 1995). Reelin had been shown to be required for the detachment of migrating neuroblasts from the RMS into the OB (Hack et al. 2002) and be able to activate PI3K-Akt-mTor pathway regulating dendritic growth (Jossin and Goffinet 2007). Disruption of ApoER2/VLDL, the receptors for reelin and Dab1, the downstream adapter protein (Andrade et al. 2007) showed a similar SVZ expansion associated with premature termination of tangential migration.

\section{Pten and Brain Tumorigenesis}

\section{Cooperativity between Pten and other tumor suppressors}

Neural stem/progenitor cells are one of the largest proliferative populations in the brain throughout life and are hypothesized as cells of origin for brain tumors (Sanai et al. 2005). In this study, deletion of Pten in the neural stem/progenitor cells in the SVZ of adult mice resulted in a dramatic expansion of the SVZ and RMS mainly due to early termination and ectopic differentiation with no progressive hyperplasia or malignant tumor formation. Similarly, the deletion of Pten in neural stem/progenitor cells in the developing postnatal brain only caused a very restricted hyper-proliferation around blood vessels in the cerebellum but not full-blown malignant tumor. These data indicate that 
Pten loss of function alone is not sufficient for malignant transformation of the neural stem/progenitor cells in the absence of other gene mutations. This is consistent with patient data that although PTEN mutation is commonly found in $15-40 \%$ of GBMs, it rarely occurs in the low-grade gliomas (Ohgaki and Kleihues 2009). This could be due to the cell cycle arrest, senescence or apoptosis induced by Pten loss sensed by cells as an oncogenic stress similar to Ras and c-Myc activation mutations. Future studies need to address whether Pten deletion can indeed induce p53, p16 ${ }^{\text {Ink4a }}$ or $\mathrm{p} 19^{\text {Arf }}$ in the neural stem/progenitor cells whose tumor suppression had been shown to restrain tumor formation in other cell types (Chen et al. 2005; Lee et al. 2011). Neverthless, we are able to generate high-grade astrocytomas arising from the SVZ when co-deleting Pten and Trp53 in adult brain (see Appendix). In the developing brain, we could generate highgrade gliomas as well as medulloblastomas in Nestin-CreER ${ }^{\text {T2 }} ;$ Pten $^{\text {loxP/loxP }} ; \operatorname{Trp} 53^{\text {loxP/loxP }}$ mice, or highly infiltrative gliomas in Nestin-CreER ${ }^{T 2} ; P_{t e n}{ }^{\text {loxP/loxP }} ; \operatorname{Trp} 53^{\text {loxP/wt }}$ mice (see Appendix). However, co-deletion of Pten and $C d k n 2 a$, another commonly mutated gene locus in human gliomas encoding $\mathrm{p} 16^{\text {Ink4a }}$ and $\mathrm{p} 19^{\text {Arf }}$, is not effective in generating any brain tumor in either mature or developing brains (data not shown). This indicates different cooperativities between Pten and other tumor suppressor pathways and tissuespecific cooperativity between Pten and $\mathrm{p} 16^{\text {Ink4a }}$ or $\mathrm{p} 19^{\text {Arf }}$ pathways.

\section{Unified function of Pten in neurogenesis and medulloblastoma genesis?}

One striking similarity between Pten ${ }^{c K O}$ neuroblast in the SVZ and Pten;Trp53 $\mathrm{dKO}$ medulloblastoma cell is that both of them had extensive neuronal differentiation. If we envision medulloblastoma genesis as a perturbed uncontrollable neurogenesis, this suggests Pten might have a similar role in normal neurogenesis and medulloblastoma genesis, that is to negatively regulate self-renewal and proliferation of neural or cancer stem cells and to keep the committed neuronal progenitors, either normal or cancerous, in an undifferentiated status. If this is true, does Pten have a similar role in the genesis of other brain tumor?

\section{Why is the perivascular niche only seen in the cerebellum?}

Even though the Nestin-CreER ${ }^{T 2}$ targeted neural stem/progenitor cells throughout the developing brain, we only observed perivascular proliferative lesions in the cerebellum when deleting Pten at birth. There are multiple possibilities to explain this finding. Firstly, cerebellum lags behind other parts of brain during development which might provide a permissive environment for maintaining the perivascular niche. Secondly, there might be more primitive stem/progenitors only present during early development which had receded in regions other than cerebellum by birth. Supporting this idea, we saw less or no perivascular proliferative lesions when deleting Pten at later postnatal days. Thirdly, it is possible Nestin-CreER ${ }^{T 2}$ target certain cell types only in the cerebellum. Future study needs to carefully compare the difference in the cell types targeted by Cre in different parts of the brain. 


\section{Cells of origin of brain tumors}

Different brain tumors might arise from different cells of origin. The Shh subtype of medulloblastoma might arise from the granule cell precursors of the cerebellar EGL whereas the Wnt subtype of medulloblastoma might develop from cells of dorsal brain stem (Gilbertson and Ellison 2008; Gibson et al. 2010). Although gliomas are hypothesized to originate from neural stem cells or glial progenitors, gliomas can arise from within or outside of proliferative niches in patients and mouse models (CBTRUS 2010; Chow et al. 2011). In this study, tumors developed throughout the brain including the OB, cortex, SVZ, thalamus, hypothalamus, brain stem and cerebellum etc. This indicates that besides neural stem/progenitors in the germinal niches such as the SVZ, SGZ and EGL, cells outside of the proliferative niches could also serve as cells of origin. The other possibilities include NG2 cells (the oligodendrocyte progenitors present in both developing and mature brain)(Trotter et al. 2009) and cells associated with blood vessels Because these two cell types are found to be the most common proliferative cells outside of germinal niches (Dawson et al. 2003) if we think the proliferative cells are more susceptible to malignant transformation. Future study could introduce common genetic lesions found in patients into the specific cell types to determine their potential as cells of origin for brain tumors. In this study, we also found that the same genetic mutations could initiate different brain tumors, medulloblastoma or gliomas, developing from different brain regions, indicating that cells of origin also determine the histological types of brain tumors.

\section{Future directions for Pten;Trp53 double knock-out medulloblastoma}

Since the Pten;Trp53 double knock-out medulloblastoma exhibit a growth pattern centering around blood vessels, it will be interesting to determine whether they initiated from certain cells around blood vessels by checking early timepoints when the tumor just starts to grow. If so, what is the exact cell associated with the blood vessels that give rise to the medulloblastoma? Why do the tumor cells adjacent to the blood vessels show a primitive and less neuronal differentiation? What is the status of Shh pathway in the tumor cells immediately adjacent to the blood vessels compared to those moving away from the blood vessels? How does this medulloblastoma compare to human medulloblastoma harboring both PTEN and TP53 mutations, and to other existing mouse medulloblastoma models?

\section{Closing summary}

Overall, our study on Pten function in the context of both development and tumorigenesis show Pten regulates appropriate positioning of terminal neuronal differentiation by keeping neuroblasts in an undifferentiated state. Undifferentiated neuroblasts can still maintain the directional migration without Pten. Pten is required for preventing ectopic perivascular proliferative niche and medulloblastoma formation. 


\section{LIST OF REFERENCES}

Abel, T.W., Baker, S.J., Fraser, M.M., Tihan, T., Nelson, J.S., Yachnis, A.T., Bouffard, J.P., Mena, H., Burger, P.C., and Eberhart, C.G. 2005. Lhermitte-Duclos disease: a report of 31 cases with immunohistochemical analysis of the PTEN/AKT/mTOR pathway. J Neuropathol Exp Neurol 64(4): 341-349.

ACS. 2010. Cancer Facts \& Figures 2010. American Cancer Society, Atlanta.

Aguirre, A., Rubio, M.E., and Gallo, V. 2010. Notch and EGFR pathway interaction regulates neural stem cell number and self-renewal. Nature 467(7313): 323-327.

Alberti, S., Krause, S.M., Kretz, O., Philippar, U., Lemberger, T., Casanova, E., Wiebel, F.F., Schwarz, H., Frotscher, M., Schutz, G. et al. 2005. Neuronal migration in the murine rostral migratory stream requires serum response factor. Proc Natl Acad Sci U S A 102(17): 6148-6153.

Alcantara Llaguno, S., Chen, J., Kwon, C.H., Jackson, E.L., Li, Y., Burns, D.K., Alvarez-Buylla, A., and Parada, L.F. 2009. Malignant astrocytomas originate from neural stem/progenitor cells in a somatic tumor suppressor mouse model. Cancer Cell 15(1): 45-56.

Alessi, D.R., James, S.R., Downes, C.P., Holmes, A.B., Gaffney, P.R., Reese, C.B., and Cohen, P. 1997. Characterization of a 3-phosphoinositide-dependent protein kinase which phosphorylates and activates protein kinase Balpha. Curr Biol 7(4): 261-269.

Alvarez-Buylla, A. and Garcia-Verdugo, J.M. 2002. Neurogenesis in adult subventricular zone. J Neurosci 22(3): 629-634.

Alvarez-Buylla, A., Kohwi, M., Nguyen, T.M., and Merkle, F.T. 2008. The heterogeneity of adult neural stem cells and the emerging complexity of their niche. Cold Spring Harb Symp Quant Biol 73: 357-365.

Andrade, N., Komnenovic, V., Blake, S.M., Jossin, Y., Howell, B., Goffinet, A., Schneider, W.J., and Nimpf, J. 2007. ApoER2/VLDL receptor and Dab1 in the rostral migratory stream function in postnatal neuronal migration independently of Reelin. Proc Natl Acad Sci U S A 104(20): 8508-8513.

Askew, D.S., Ashmun, R.A., Simmons, B.C., and Cleveland, J.L. 1991. Constitutive cmyc expression in an IL-3-dependent myeloid cell line suppresses cell cycle arrest and accelerates apoptosis. Oncogene 6(10): 1915-1922.

Backman, S.A., Stambolic, V., Suzuki, A., Haight, J., Elia, A., Pretorius, J., Tsao, M.S., Shannon, P., Bolon, B., Ivy, G.O. et al. 2001. Deletion of Pten in mouse brain causes seizures, ataxia and defects in soma size resembling Lhermitte-Duclos disease. Nat Genet 29(4): 396-403. 
Baeza, N., Masuoka, J., Kleihues, P., and Ohgaki, H. 2003. AXIN1 mutations but not deletions in cerebellar medulloblastomas. Oncogene 22(4): 632-636.

Bajenaru, M.L., Hernandez, M.R., Perry, A., Zhu, Y., Parada, L.F., Garbow, J.R., and Gutmann, D.H. 2003. Optic nerve glioma in mice requires astrocyte Nfl gene inactivation and Nf1 brain heterozygosity. Cancer Res 63(24): 8573-8577.

Ben-Arie, N., Bellen, H.J., Armstrong, D.L., McCall, A.E., Gordadze, P.R., Guo, Q., Matzuk, M.M., and Zoghbi, H.Y. 1997. Math1 is essential for genesis of cerebellar granule neurons. Nature 390(6656): 169-172.

Boutin, C., Diestel, S., Desoeuvre, A., Tiveron, M.C., and Cremer, H. 2008. Efficient in vivo electroporation of the postnatal rodent forebrain. PLoS One 3(4): e1883.

Calabrese, C., Poppleton, H., Kocak, M., Hogg, T.L., Fuller, C., Hamner, B., Oh, E.Y., Gaber, M.W., Finklestein, D., Allen, M. et al. 2007. A perivascular niche for brain tumor stem cells. Cancer Cell 11(1): 69-82.

Campbell, D.S. and Holt, C.E. 2001. Chemotropic responses of retinal growth cones mediated by rapid local protein synthesis and degradation. Neuron 32(6): 10131026.

Carletti, B. and Rossi, F. 2008. Neurogenesis in the cerebellum. Neuroscientist 14(1): 91-100.

Castellino, R.C., Barwick, B.G., Schniederjan, M., Buss, M.C., Becher, O., Hambardzumyan, D., Macdonald, T.J., Brat, D.J., and Durden, D.L. 2010. Heterozygosity for Pten promotes tumorigenesis in a mouse model of medulloblastoma. PLoS One 5(5): e10849.

CBTRUS. 2010. CBTRUS statistical report: primary brain and central nervous system tumors diagnosed in the United States in 2004-2006. Central Brain Tumor Registry of the United States, Hinsdale.

Chadborn, N.H., Ahmed, A.I., Holt, M.R., Prinjha, R., Dunn, G.A., Jones, G.E., and Eickholt, B.J. 2006. PTEN couples Sema3A signalling to growth cone collapse. J Cell Sci 119(Pt 5): 951-957.

Chalhoub, N. and Baker, S.J. 2009. PTEN and the PI3-kinase pathway in cancer. Annu Rev Pathol 4: 127-150.

Chen, Z., Trotman, L.C., Shaffer, D., Lin, H.K., Dotan, Z.A., Niki, M., Koutcher, J.A., Scher, H.I., Ludwig, T., Gerald, W. et al. 2005. Crucial role of p53-dependent cellular senescence in suppression of Pten-deficient tumorigenesis. Nature 436(7051): 725-730. 
Chesler, A.T., Le Pichon, C.E., Brann, J.H., Araneda, R.C., Zou, D.J., and Firestein, S. 2008. Selective gene expression by postnatal electroporation during olfactory interneuron nurogenesis. PLoS One 3(1): e1517.

Cho, Y.J., Tsherniak, A., Tamayo, P., Santagata, S., Ligon, A., Greulich, H., Berhoukim, R., Amani, V., Goumnerova, L., Eberhart, C.G. et al. 2011. Integrative genomic analysis of medulloblastoma identifies a molecular subgroup that drives poor clinical outcome. J Clin Oncol 29(11): 1424-1430.

Choo, A.Y., Yoon, S.O., Kim, S.G., Roux, P.P., and Blenis, J. 2008. Rapamycin differentially inhibits $\mathrm{S} 6 \mathrm{Ks}$ and 4E-BP1 to mediate cell-type-specific repression of mRNA translation. Proc Natl Acad Sci U S A 105(45): 17414-17419.

Chow, D.K., Groszer, M., Pribadi, M., Machniki, M., Carmichael, S.T., Liu, X., and Trachtenberg, J.T. 2009. Laminar and compartmental regulation of dendritic growth in mature cortex. Nat Neurosci 12(2): 116-118.

Chow, L.M., Endersby, R., Zhu, X., Rankin, S., Qu, C., Zhang, J., Broniscer, A., Ellison, D.W., and Baker, S.J. 2011. Cooperativity within and among Pten, p53, and $\mathrm{Rb}$ pathways induces high-grade astrocytoma in adult brain. Cancer Cell 19(3): 305-316.

Cicero, S.A., Johnson, D., Reyntjens, S., Frase, S., Connell, S., Chow, L.M., Baker, S.J., Sorrentino, B.P., and Dyer, M.A. 2009. Cells previously identified as retinal stem cells are pigmented ciliary epithelial cells. Proc Natl Acad Sci U S A 106(16): 6685-6690.

Coskun, V., Wu, H., Blanchi, B., Tsao, S., Kim, K., Zhao, J., Biancotti, J.C., Hutnick, L., Krueger, R.C., Jr., Fan, G. et al. 2008. CD133+ neural stem cells in the ependyma of mammalian postnatal forebrain. Proc Natl Acad Sci U S A 105(3): 1026-1031.

Cotter, L., Ozcelik, M., Jacob, C., Pereira, J.A., Locher, V., Baumann, R., Relvas, J.B., Suter, U., and Tricaud, N. 2010. Dlg1-PTEN interaction regulates myelin thickness to prevent damaging peripheral nerve overmyelination. Science 328(5984): 1415-1418.

Curtis, M.A., Kam, M., Nannmark, U., Anderson, M.F., Axell, M.Z., Wikkelso, C., Holtas, S., van Roon-Mom, W.M., Bjork-Eriksson, T., Nordborg, C. et al. 2007. Human neuroblasts migrate to the olfactory bulb via a lateral ventricular extension. Science 315(5816): 1243-1249.

D'Arcangelo, G., Miao, G.G., Chen, S.C., Soares, H.D., Morgan, J.I., and Curran, T. 1995. A protein related to extracellular matrix proteins deleted in the mouse mutant reeler. Nature 374(6524): 719-723. 
Dahmane, N. and Ruiz i Altaba, A. 1999. Sonic hedgehog regulates the growth and patterning of the cerebellum. Development 126(14): 3089-3100.

Das, S., Dixon, J.E., and Cho, W. 2003. Membrane-binding and activation mechanism of PTEN. Proc Natl Acad Sci U S A 100(13): 7491-7496.

Dawson, M.R., Polito, A., Levine, J.M., and Reynolds, R. 2003. NG2-expressing glial progenitor cells: an abundant and widespread population of cycling cells in the adult rat CNS. Mol Cell Neurosci 24(2): 476-488.

Di Cristofano, A., Pesce, B., Cordon-Cardo, C., and Pandolfi, P.P. 1998. Pten is essential for embryonic development and tumour suppression. Nat Genet 19(4): 348-355.

Doetsch, F., Caille, I., Lim, D.A., Garcia-Verdugo, J.M., and Alvarez-Buylla, A. 1999. Subventricular zone astrocytes are neural stem cells in the adult mammalian brain. Cell 97(6): 703-716.

Doetsch, F., Petreanu, L., Caille, I., Garcia-Verdugo, J.M., and Alvarez-Buylla, A. 2002. EGF converts transit-amplifying neurogenic precursors in the adult brain into multipotent stem cells. Neuron 36(6): 1021-1034.

Dowling, R.J., Topisirovic, I., Alain, T., Bidinosti, M., Fonseca, B.D., Petroulakis, E., Wang, X., Larsson, O., Selvaraj, A., Liu, Y. et al. 2010. mTORC1-mediated cell proliferation, but not cell growth, controlled by the 4E-BPs. Science 328(5982): 1172-1176.

Eng, C. 2003. PTEN: one gene, many syndromes. Hum Mutat 22(3): 183-198.

Engelman, J.A., Luo, J., and Cantley, L.C. 2006. The evolution of phosphatidylinositol 3-kinases as regulators of growth and metabolism. Nat Rev Genet 7(8): 606-619.

Feil, R., Brocard, J., Mascrez, B., LeMeur, M., Metzger, D., and Chambon, P. 1996. Ligand-activated site-specific recombination in mice. Proc Natl Acad Sci U S A 93(20): 10887-10890.

Feil, R., Wagner, J., Metzger, D., and Chambon, P. 1997. Regulation of Cre recombinase activity by mutated estrogen receptor ligand-binding domains. Biochem Biophys Res Commun 237(3): 752-757.

Ferguson, G.J., Milne, L., Kulkarni, S., Sasaki, T., Walker, S., Andrews, S., Crabbe, T., Finan, P., Jones, G., Jackson, S. et al. 2007. PI(3)Kgamma has an important context-dependent role in neutrophil chemokinesis. Nat Cell Biol 9(1): 86-91.

Frank, A.J., Hernan, R., Hollander, A., Lindsey, J.C., Lusher, M.E., Fuller, C.E., Clifford, S.C., and Gilbertson, R.J. 2004. The TP53-ARF tumor suppressor pathway is frequently disrupted in large/cell anaplastic medulloblastoma. Brain Res Mol Brain Res 121(1-2): 137-140. 
Frappart, P.O., Lee, Y., Russell, H.R., Chalhoub, N., Wang, Y.D., Orii, K.E., Zhao, J., Kondo, N., Baker, S.J., and McKinnon, P.J. 2009. Recurrent genomic alterations characterize medulloblastoma arising from DNA double-strand break repair deficiency. Proc Natl Acad Sci U S A 106(6): 1880-1885.

Frappart, P.O. and McKinnon, P.J. 2008. Mouse models of DNA double-strand break repair and neurological disease. DNA Repair (Amst) 7(7): 1051-1060.

Fraser, M.M., Bayazitov, I.T., Zakharenko, S.S., and Baker, S.J. 2008. Phosphatase and tensin homolog, deleted on chromosome 10 deficiency in brain causes defects in synaptic structure, transmission and plasticity, and myelination abnormalities. Neuroscience 151(2): 476-488.

Fraser, M.M., Zhu, X., Kwon, C.H., Uhlmann, E.J., Gutmann, D.H., and Baker, S.J. 2004. Pten loss causes hypertrophy and increased proliferation of astrocytes in vivo. Cancer Res 64(21): 7773-7779.

Funamoto, S., Meili, R., Lee, S., Parry, L., and Firtel, R.A. 2002. Spatial and temporal regulation of 3-phosphoinositides by PI 3-kinase and PTEN mediates chemotaxis. Cell 109(5): 611-623.

Furnari, F.B., Fenton, T., Bachoo, R.M., Mukasa, A., Stommel, J.M., Stegh, A., Hahn, W.C., Ligon, K.L., Louis, D.N., Brennan, C. et al. 2007. Malignant astrocytic glioma: genetics, biology, and paths to treatment. Genes Dev 21(21): 2683-2710.

Furnari, F.B., Huang, H.J., and Cavenee, W.K. 1998. The phosphoinositol phosphatase activity of PTEN mediates a serum-sensitive G1 growth arrest in glioma cells. Cancer Res 58(22): 5002-5008.

Gage, F.H. 2002. Neurogenesis in the adult brain. $J$ Neurosci 22(3): 612-613.

Georgescu, M.M., Kirsch, K.H., Akagi, T., Shishido, T., and Hanafusa, H. 1999. The tumor-suppressor activity of PTEN is regulated by its carboxyl-terminal region. Proc Natl Acad Sci U S A 96(18): 10182-10187.

Georgescu, M.M., Kirsch, K.H., Kaloudis, P., Yang, H., Pavletich, N.P., and Hanafusa, H. 2000. Stabilization and productive positioning roles of the C2 domain of PTEN tumor suppressor. Cancer Res 60(24): 7033-7038.

Ghashghaei, H.T., Lai, C., and Anton, E.S. 2007. Neuronal migration in the adult brain: are we there yet? Nat Rev Neurosci 8(2): 141-151.

Gibson, P., Tong, Y., Robinson, G., Thompson, M.C., Currle, D.S., Eden, C., Kranenburg, T.A., Hogg, T., Poppleton, H., Martin, J. et al. 2010. Subtypes of medulloblastoma have distinct developmental origins. Nature 468(7327): 10951099. 
Gilbertson, R.J. and Clifford, S.C. 2003. PDGFRB is overexpressed in metastatic medulloblastoma. Nat Genet 35(3): 197-198.

Gilbertson, R.J. and Ellison, D.W. 2008. The origins of medulloblastoma subtypes. Annu Rev Pathol 3: 341-365.

Giri, D. and Ittmann, M. 1999. Inactivation of the PTEN tumor suppressor gene is associated with increased angiogenesis in clinically localized prostate carcinoma. Hum Pathol 30(4): 419-424.

Goebbels, S., Oltrogge, J.H., Kemper, R., Heilmann, I., Bormuth, I., Wolfer, S., Wichert, S.P., Mobius, W., Liu, X., Lappe-Siefke, C. et al. 2010. Elevated phosphatidylinositol 3,4,5-trisphosphate in glia triggers cell-autonomous membrane wrapping and myelination. J Neurosci 30(26): 8953-8964.

Goffin, A., Hoefsloot, L.H., Bosgoed, E., Swillen, A., and Fryns, J.P. 2001. PTEN mutation in a family with Cowden syndrome and autism. Am J Med Genet 105(6): 521-524.

Goldowitz, D. and Hamre, K. 1998. The cells and molecules that make a cerebellum. Trends Neurosci 21(9): 375-382.

Goodrich, L.V., Milenkovic, L., Higgins, K.M., and Scott, M.P. 1997. Altered neural cell fates and medulloblastoma in mouse patched mutants. Science 277(5329): 11091113.

Gottschalk, A.R., Basila, D., Wong, M., Dean, N.M., Brandts, C.H., Stokoe, D., and Haas-Kogan, D.A. 2001. p27Kip1 is required for PTEN-induced G1 growth arrest. Cancer Res 61(5): 2105-2111.

Gregorian, C., Nakashima, J., Le Belle, J., Ohab, J., Kim, R., Liu, A., Smith, K.B., Groszer, M., Garcia, A.D., Sofroniew, M.V. et al. 2009. Pten deletion in adult neural stem/progenitor cells enhances constitutive neurogenesis. $J$ Neurosci 29(6): 1874-1886.

Groszer, M., Erickson, R., Scripture-Adams, D.D., Dougherty, J.D., Le Belle, J., Zack, J.A., Geschwind, D.H., Liu, X., Kornblum, H.I., and Wu, H. 2006. PTEN negatively regulates neural stem cell self-renewal by modulating G0-G1 cell cycle entry. Proc Natl Acad Sci U S A 103(1): 111-116.

Groszer, M., Erickson, R., Scripture-Adams, D.D., Lesche, R., Trumpp, A., Zack, J.A., Kornblum, H.I., Liu, X., and $\mathrm{Wu}$, H. 2001. Negative regulation of neural stem/progenitor cell proliferation by the Pten tumor suppressor gene in vivo. Science 294(5549): 2186-2189. 
Guerrero-Cazares, H., Gonzalez-Perez, O., Soriano-Navarro, M., Zamora-Berridi, G., Garcia-Verdugo, J.M., and Quinones-Hinojosa, A. 2011. Cytoarchitecture of the lateral ganglionic eminence and rostral extension of the lateral ventricle in the human fetal brain. J Comp Neurol 519(6): 1165-1180.

Hack, I., Bancila, M., Loulier, K., Carroll, P., and Cremer, H. 2002. Reelin is a detachment signal in tangential chain-migration during postnatal neurogenesis. Nat Neurosci 5(10): 939-945.

Hahn, H., Wojnowski, L., Specht, K., Kappler, R., Calzada-Wack, J., Potter, D., Zimmer, A., Muller, U., Samson, E., and Quintanilla-Martinez, L. 2000. Patched target Igf 2 is indispensable for the formation of medulloblastoma and rhabdomyosarcoma. J Biol Chem 275(37): 28341-28344.

Hambardzumyan, D., Becher, O.J., Rosenblum, M.K., Pandolfi, P.P., ManovaTodorova, K., and Holland, E.C. 2008. PI3K pathway regulates survival of cancer stem cells residing in the perivascular niche following radiation in medulloblastoma in vivo. Genes Dev 22(4): 436-448.

Harrington, E.P., Zhao, C., Fancy, S.P., Kaing, S., Franklin, R.J., and Rowitch, D.H. 2010. Oligodendrocyte PTEN is required for myelin and axonal integrity, not remyelination. Ann Neurol 68(5): 703-716.

Harrington, L.S., Findlay, G.M., Gray, A., Tolkacheva, T., Wigfield, S., Rebholz, H., Barnett, J., Leslie, N.R., Cheng, S., Shepherd, P.R. et al. 2004. The TSC1-2 tumor suppressor controls insulin-PI3K signaling via regulation of IRS proteins. $J$ Cell Biol 166(2): 213-223.

Hartmann, W., Digon-Sontgerath, B., Koch, A., Waha, A., Endl, E., Dani, I., Denkhaus, D., Goodyer, C.G., Sorensen, N., Wiestler, O.D. et al. 2006. Phosphatidylinositol 3'-kinase/AKT signaling is activated in medulloblastoma cell proliferation and is associated with reduced expression of PTEN. Clin Cancer Res 12(10): 30193027.

Heit, B., Robbins, S.M., Downey, C.M., Guan, Z., Colarusso, P., Miller, B.J., Jirik, F.R., and Kubes, P. 2008. PTEN functions to 'prioritize' chemotactic cues and prevent 'distraction' in migrating neutrophils. Nat Immunol 9(7): 743-752.

Hernan, R., Fasheh, R., Calabrese, C., Frank, A.J., Maclean, K.H., Allard, D., Barraclough, R., and Gilbertson, R.J. 2003. ERBB2 up-regulates S100A4 and several other prometastatic genes in medulloblastoma. Cancer Res 63(1): 140148.

Hoeller, O. and Kay, R.R. 2007. Chemotaxis in the absence of PIP3 gradients. Curr Biol 17(9): 813-817. 
Hoshino, M., Nakamura, S., Mori, K., Kawauchi, T., Terao, M., Nishimura, Y.V., Fukuda, A., Fuse, T., Matsuo, N., Sone, M. et al. 2005. Ptfla, a bHLH transcriptional gene, defines GABAergic neuronal fates in cerebellum. Neuron 47(2): 201-213.

Huang, H., Mahler-Araujo, B.M., Sankila, A., Chimelli, L., Yonekawa, Y., Kleihues, P., and Ohgaki, H. 2000. APC mutations in sporadic medulloblastomas. Am J Pathol 156(2): 433-437.

Huang, S., Bjornsti, M.A., and Houghton, P.J. 2003. Rapamycins: mechanism of action and cellular resistance. Cancer Biol Ther 2(3): 222-232.

Huard, J.M., Forster, C.C., Carter, M.L., Sicinski, P., and Ross, M.E. 1999. Cerebellar histogenesis is disturbed in mice lacking cyclin D2. Development 126(9): 19271935.

Ihrie, R.A. and Alvarez-Buylla, A. 2008. Cells in the astroglial lineage are neural stem cells. Cell Tissue Res 331(1): 179-191.

Iijima, M. and Devreotes, P. 2002. Tumor suppressor PTEN mediates sensing of chemoattractant gradients. Cell 109(5): 599-610.

Inoki, K., Corradetti, M.N., and Guan, K.L. 2005. Dysregulation of the TSC-mTOR pathway in human disease. Nat Genet 37(1): 19-24.

Jacques, T.S., Swales, A., Brzozowski, M.J., Henriquez, N.V., Linehan, J.M., Mirzadeh, Z., O'Malley, C., Naumann, H., Alvarez-Buylla, A., and Brandner, S. 2010. Combinations of genetic mutations in the adult neural stem cell compartment determine brain tumour phenotypes. EMBO J 29(1): 222-235.

Jaworski, J., Spangler, S., Seeburg, D.P., Hoogenraad, C.C., and Sheng, M. 2005. Control of dendritic arborization by the phosphoinositide-3'-kinase-Akt-mammalian target of rapamycin pathway. J Neurosci 25(49): 11300-11312.

Jiang, B.H., Zheng, J.Z., Aoki, M., and Vogt, P.K. 2000. Phosphatidylinositol 3-kinase signaling mediates angiogenesis and expression of vascular endothelial growth factor in endothelial cells. Proc Natl Acad Sci U S A 97(4): 1749-1753.

Jonkers, J., Meuwissen, R., van der Gulden, H., Peterse, H., van der Valk, M., and Berns, A. 2001. Synergistic tumor suppressor activity of BRCA2 and p53 in a conditional mouse model for breast cancer. Nat Genet 29(4): 418-425.

Jossin, Y. and Goffinet, A.M. 2007. Reelin signals through phosphatidylinositol 3-kinase and Akt to control cortical development and through mTor to regulate dendritic growth. Mol Cell Biol 27(20): 7113-7124. 
Kenney, A.M., Widlund, H.R., and Rowitch, D.H. 2004. Hedgehog and PI-3 kinase signaling converge on Nmyc1 to promote cell cycle progression in cerebellar neuronal precursors. Development 131(1): 217-228.

Kim, Y., Comte, I., Szabo, G., Hockberger, P., and Szele, F.G. 2009. Adult mouse subventricular zone stem and progenitor cells are sessile and epidermal growth factor receptor negatively regulates neuroblast migration. PLoS One 4(12): e8122.

Knoepfler, P.S., Cheng, P.F., and Eisenman, R.N. 2002. N-myc is essential during neurogenesis for the rapid expansion of progenitor cell populations and the inhibition of neuronal differentiation. Genes Dev 16(20): 2699-2712.

Kohwi, M., Osumi, N., Rubenstein, J.L., and Alvarez-Buylla, A. 2005. Pax6 is required for making specific subpopulations of granule and periglomerular neurons in the olfactory bulb. J Neurosci 25(30): 6997-7003.

Kohwi, M., Petryniak, M.A., Long, J.E., Ekker, M., Obata, K., Yanagawa, Y., Rubenstein, J.L., and Alvarez-Buylla, A. 2007. A subpopulation of olfactory bulb GABAergic interneurons is derived from Emx1- and Dlx5/6-expressing progenitors. J Neurosci 27(26): 6878-6891.

Kokovay, E., Goderie, S., Wang, Y., Lotz, S., Lin, G., Sun, Y., Roysam, B., Shen, Q., and Temple, S. 2010. Adult SVZ lineage cells home to and leave the vascular niche via differential responses to SDF1/CXCR4 signaling. Cell Stem Cell 7(2): 163-173.

Kolsch, V., Charest, P.G., and Firtel, R.A. 2008. The regulation of cell motility and chemotaxis by phospholipid signaling. J Cell Sci 121(Pt 5): 551-559.

Kool, M., Koster, J., Bunt, J., Hasselt, N.E., Lakeman, A., van Sluis, P., Troost, D., Meeteren, N.S., Caron, H.N., Cloos, J. et al. 2008. Integrated genomics identifies five medulloblastoma subtypes with distinct genetic profiles, pathway signatures and clinicopathological features. PLoS One 3(8): e3088.

Koul, D., Parthasarathy, R., Shen, R., Davies, M.A., Jasser, S.A., Chintala, S.K., Rao, J.S., Sun, Y., Benvenisite, E.N., Liu, T.J. et al. 2001. Suppression of matrix metalloproteinase-2 gene expression and invasion in human glioma cells by MMAC/PTEN. Oncogene 20(46): 6669-6678.

Kubiatowski, T., Jang, T., Lachyankar, M.B., Salmonsen, R., Nabi, R.R., Quesenberry, P.J., Litofsky, N.S., Ross, A.H., and Recht, L.D. 2001. Association of increased phosphatidylinositol 3-kinase signaling with increased invasiveness and gelatinase activity in malignant gliomas. J Neurosurg 95(3): 480-488.

Kumar, V., Zhang, M.X., Swank, M.W., Kunz, J., and Wu, G.Y. 2005. Regulation of dendritic morphogenesis by Ras-PI3K-Akt-mTOR and Ras-MAPK signaling pathways. J Neurosci 25(49): 11288-11299. 
Kwon, C.H., Luikart, B.W., Powell, C.M., Zhou, J., Matheny, S.A., Zhang, W., Li, Y., Baker, S.J., and Parada, L.F. 2006. Pten regulates neuronal arborization and social interaction in mice. Neuron 50(3): 377-388.

Kwon, C.H., Zhao, D., Chen, J., Alcantara, S., Li, Y., Burns, D.K., Mason, R.P., Lee, E.Y., Wu, H., and Parada, L.F. 2008. Pten haploinsufficiency accelerates formation of high-grade astrocytomas. Cancer Res 68(9): 3286-3294.

Kwon, C.H., Zhu, X., Zhang, J., and Baker, S.J. 2003. mTor is required for hypertrophy of Pten-deficient neuronal soma in vivo. Proc Natl Acad Sci U S A 100(22): 12923-12928.

Kwon, C.H., Zhu, X., Zhang, J., Knoop, L.L., Tharp, R., Smeyne, R.J., Eberhart, C.G., Burger, P.C., and Baker, S.J. 2001. Pten regulates neuronal soma size: a mouse model of Lhermitte-Duclos disease. Nat Genet 29(4): 404-411.

Lacalle, R.A., Gomez-Mouton, C., Barber, D.F., Jimenez-Baranda, S., Mira, E., Martinez, A.C., Carrera, A.C., and Manes, S. 2004. PTEN regulates motility but not directionality during leukocyte chemotaxis. J Cell Sci 117(Pt 25): 6207-6215.

Lee, A., Kessler, J.D., Read, T.A., Kaiser, C., Corbeil, D., Huttner, W.B., Johnson, J.E., and Wechsler-Reya, R.J. 2005. Isolation of neural stem cells from the postnatal cerebellum. Nat Neurosci 8(6): 723-729.

Lee, J., Kotliarova, S., Kotliarov, Y., Li, A., Su, Q., Donin, N.M., Pastorino, S., Purow, B.W., Christopher, N., Zhang, W. et al. 2006a. Tumor stem cells derived from glioblastomas cultured in bFGF and EGF more closely mirror the phenotype and genotype of primary tumors than do serum-cultured cell lines. Cancer Cell 9(5): 391-403.

Lee, J.O., Yang, H., Georgescu, M.M., Di Cristofano, A., Maehama, T., Shi, Y., Dixon, J.E., Pandolfi, P., and Pavletich, N.P. 1999. Crystal structure of the PTEN tumor suppressor: implications for its phosphoinositide phosphatase activity and membrane association. Cell 99(3): 323-334.

Lee, J.Y., Nakada, D., Yilmaz, O.H., Tothova, Z., Joseph, N.M., Lim, M.S., Gilliland, D.G., and Morrison, S.J. 2011. mTOR activation induces tumor suppressors that inhibit leukemogenesis and deplete hematopoietic stem cells after Pten deletion. Cell Stem Cell 7(5): 593-605.

Lee, Y. and McKinnon, P.J. 2002. DNA ligase IV suppresses medulloblastoma formation. Cancer Res 62(22): 6395-6399.

Lee, Y., Miller, H.L., Russell, H.R., Boyd, K., Curran, T., and McKinnon, P.J. 2006 b. Patched 2 modulates tumorigenesis in patched 1 heterozygous mice. Cancer Res 66(14): 6964-6971. 
Lendahl, U., Zimmerman, L.B., and McKay, R.D. 1990. CNS stem cells express a new class of intermediate filament protein. Cell 60(4): 585-595.

Li, D.M. and Sun, H. 1997. TEP1, encoded by a candidate tumor suppressor locus, is a novel protein tyrosine phosphatase regulated by transforming growth factor beta. Cancer Res 57(11): 2124-2129.

Li, J., Yen, C., Liaw, D., Podsypanina, K., Bose, S., Wang, S.I., Puc, J., Miliaresis, C., Rodgers, L., McCombie, R. et al. 1997. PTEN, a putative protein tyrosine phosphatase gene mutated in human brain, breast, and prostate cancer. Science 275(5308): 1943-1947.

Lo, K.C., Rossi, M.R., Eberhart, C.G., and Cowell, J.K. 2007. Genome wide copy number abnormalities in pediatric medulloblastomas as assessed by array comparative genome hybridization. Brain Pathol 17(3): 282-296.

Lowe, S.W. and Ruley, H.E. 1993. Stabilization of the p53 tumor suppressor is induced by adenovirus 5 E1A and accompanies apoptosis. Genes Dev 7(4): 535-545.

MacDonald, T.J., Brown, K.M., LaFleur, B., Peterson, K., Lawlor, C., Chen, Y., Packer, R.J., Cogen, P., and Stephan, D.A. 2001. Expression profiling of medulloblastoma: PDGFRA and the RAS/MAPK pathway as therapeutic targets for metastatic disease. Nat Genet 29(2): 143-152.

Maehama, T. and Dixon, J.E. 1998. The tumor suppressor, PTEN/MMAC1, dephosphorylates the lipid second messenger, phosphatidylinositol 3,4,5trisphosphate. J Biol Chem 273(22): 13375-13378.

Marino, S., Krimpenfort, P., Leung, C., van der Korput, H.A., Trapman, J., Camenisch, I., Berns, A., and Brandner, S. 2002. PTEN is essential for cell migration but not for fate determination and tumourigenesis in the cerebellum. Development 129(14): 3513-3522.

McCabe, M.G., Ichimura, K., Liu, L., Plant, K., Backlund, L.M., Pearson, D.M., and Collins, V.P. 2006. High-resolution array-based comparative genomic hybridization of medulloblastomas and supratentorial primitive neuroectodermal tumors. J Neuropathol Exp Neurol 65(6): 549-561.

Meikle, L., Talos, D.M., Onda, H., Pollizzi, K., Rotenberg, A., Sahin, M., Jensen, F.E., and Kwiatkowski, D.J. 2007. A mouse model of tuberous sclerosis: neuronal loss of Tsc1 causes dysplastic and ectopic neurons, reduced myelination, seizure activity, and limited survival. J Neurosci 27(21): 5546-5558.

Merkle, F.T., Mirzadeh, Z., and Alvarez-Buylla, A. 2007. Mosaic organization of neural stem cells in the adult brain. Science 317(5836): 381-384. 
Metzger, D., Clifford, J., Chiba, H., and Chambon, P. 1995. Conditional site-specific recombination in mammalian cells using a ligand-dependent chimeric Cre recombinase. Proc Natl Acad Sci U S A 92(15): 6991-6995.

Ming, G.L. and Song, H. 2005. Adult neurogenesis in the mammalian central nervous system. Annu Rev Neurosci 28: 223-250.

Mirzadeh, Z., Merkle, F.T., Soriano-Navarro, M., Garcia-Verdugo, J.M., and AlvarezBuylla, A. 2008. Neural stem cells confer unique pinwheel architecture to the ventricular surface in neurogenic regions of the adult brain. Cell Stem Cell 3(3): 265-278.

Miyazawa, K., Himi, T., Garcia, V., Yamagishi, H., Sato, S., and Ishizaki, Y. 2000. A role for $\mathrm{p} 27 / \mathrm{Kip} 1$ in the control of cerebellar granule cell precursor proliferation. J Neurosci 20(15): 5756-5763.

Myers, M.P., Stolarov, J.P., Eng, C., Li, J., Wang, S.I., Wigler, M.H., Parsons, R., and Tonks, N.K. 1997. P-TEN, the tumor suppressor from human chromosome 10q23, is a dual-specificity phosphatase. Proc Natl Acad Sci U S A 94(17): 90529057.

Nishio, M., Watanabe, K., Sasaki, J., Taya, C., Takasuga, S., Iizuka, R., Balla, T., Yamazaki, M., Watanabe, H., Itoh, R. et al. 2007. Control of cell polarity and motility by the PtdIns(3,4,5)P3 phosphatase SHIP1. Nat Cell Biol 9(1): 36-44.

Northcott, P.A., Korshunov, A., Witt, H., Hielscher, T., Eberhart, C.G., Mack, S., Bouffet, E., Clifford, S.C., Hawkins, C.E., French, P. et al. 2010. Medulloblastoma comprises four distinct molecular variants. J Clin Oncol 29(11): 1408-1414.

O'Reilly, K.E., Rojo, F., She, Q.B., Solit, D., Mills, G.B., Smith, D., Lane, H., Hofmann, F., Hicklin, D.J., Ludwig, D.L. et al. 2006. mTOR inhibition induces upstream receptor tyrosine kinase signaling and activates Akt. Cancer Res 66(3): 15001508.

Ohgaki, H. and Kleihues, P. 2007. Genetic pathways to primary and secondary glioblastoma. Am J Pathol 170(5): 1445-1453.

Ohgaki, H. and Kleihues, P. 2009. Genetic alterations and signaling pathways in the evolution of gliomas. Cancer Sci 100(12): 2235-2241.

Ono, K., Tomasiewicz, H., Magnuson, T., and Rutishauser, U. 1994. N-CAM mutation inhibits tangential neuronal migration and is phenocopied by enzymatic removal of polysialic acid. Neuron 13(3): 595-609.

Paintlia, A.S., Paintlia, M.K., Singh, A.K., Orak, J.K., and Singh, I. 2010. Activation of PPAR-gamma and PTEN cascade participates in lovastatin-mediated accelerated differentiation of oligodendrocyte progenitor cells. Glia 58(14): 1669-1685. 
Parsons, D.W., Jones, S., Zhang, X., Lin, J.C., Leary, R.J., Angenendt, P., Mankoo, P., Carter, H., Siu, I.M., Gallia, G.L. et al. 2008. An integrated genomic analysis of human glioblastoma multiforme. Science 321(5897): 1807-1812.

Parsons, D.W., Li, M., Zhang, X., Jones, S., Leary, R.J., Lin, J.C., Boca, S.M., Carter, H., Samayoa, J., Bettegowda, C. et al. 2011. The genetic landscape of the childhood cancer medulloblastoma. Science 331(6016): 435-439.

Petreanu, L. and Alvarez-Buylla, A. 2002. Maturation and death of adult-born olfactory bulb granule neurons: role of olfaction. J Neurosci 22(14): 6106-6113.

Podsypanina, K., Ellenson, L.H., Nemes, A., Gu, J., Tamura, M., Yamada, K.M., Cordon-Cardo, C., Catoretti, G., Fisher, P.E., and Parsons, R. 1999. Mutation of Pten/Mmac1 in mice causes neoplasia in multiple organ systems. Proc Natl Acad Sci U S A 96(4): 1563-1568.

Polleux, F. and Ghosh, A. 2002. The slice overlay assay: a versatile tool to study the influence of extracellular signals on neuronal development. Sci STKE 2002(136): p19.

Pore, N., Jiang, Z., Shu, H.K., Bernhard, E., Kao, G.D., and Maity, A. 2006. Akt1 activation can augment hypoxia-inducible factor-1alpha expression by increasing protein translation through a mammalian target of rapamycin-independent pathway. Mol Cancer Res 4(7): 471-479.

Quinones-Hinojosa, A., Sanai, N., Soriano-Navarro, M., Gonzalez-Perez, O., Mirzadeh, Z., Gil-Perotin, S., Romero-Rodriguez, R., Berger, M.S., Garcia-Verdugo, J.M., and Alvarez-Buylla, A. 2006. Cellular composition and cytoarchitecture of the adult human subventricular zone: a niche of neural stem cells. J Comp Neurol 494(3): 415-434.

Raffel, C., Jenkins, R.B., Frederick, L., Hebrink, D., Alderete, B., Fults, D.W., and James, C.D. 1997. Sporadic medulloblastomas contain PTCH mutations. Cancer Res 57(5): 842-845.

Raftopoulou, M., Etienne-Manneville, S., Self, A., Nicholls, S., and Hall, A. 2004. Regulation of cell migration by the $\mathrm{C} 2$ domain of the tumor suppressor PTEN. Science 303(5661): 1179-1181.

Rao, G., Pedone, C.A., Del Valle, L., Reiss, K., Holland, E.C., and Fults, D.W. 2004. Sonic hedgehog and insulin-like growth factor signaling synergize to induce medulloblastoma formation from nestin-expressing neural progenitors in mice. Oncogene 23(36): 6156-6162.

Reddy, P., Liu, L., Adhikari, D., Jagarlamudi, K., Rajareddy, S., Shen, Y., Du, C., Tang, W., Hamalainen, T., Peng, S.L. et al. 2008. Oocyte-specific deletion of Pten causes premature activation of the primordial follicle pool. Science 319(5863): 611-613. 
Reifenberger, J., Wolter, M., Weber, R.G., Megahed, M., Ruzicka, T., Lichter, P., and Reifenberger, G. 1998. Missense mutations in SMOH in sporadic basal cell carcinomas of the skin and primitive neuroectodermal tumors of the central nervous system. Cancer Res 58(9): 1798-1803.

Riegert-Johnson, D.L., Gleeson, F.C., Roberts, M., Tholen, K., Youngborg, L., Bullock, M., and Boardman, L.A. 2010. Cancer and Lhermitte-Duclos disease are common in Cowden syndrome patients. Hered Cancer Clin Pract 8(1): 6.

Riobo, N.A., Lu, K., Ai, X., Haines, G.M., and Emerson, C.P., Jr. 2006. Phosphoinositide 3-kinase and Akt are essential for Sonic Hedgehog signaling. Proc Natl Acad Sci U S A 103(12): 4505-4510.

Rosner, M., Hanneder, M., Siegel, N., Valli, A., Fuchs, C., and Hengstschlager, M. 2008. The mTOR pathway and its role in human genetic diseases. Mutat Res 659(3): 284-292.

Rossi, M.R., Conroy, J., McQuaid, D., Nowak, N.J., Rutka, J.T., and Cowell, J.K. 2006. Array CGH analysis of pediatric medulloblastomas. Genes Chromosomes Cancer 45(3): 290-303.

Rowitch, D.H. and Kriegstein, A.R. 2010. Developmental genetics of vertebrate glial-cell specification. Nature 468(7321): 214-222.

Sanai, N., Alvarez-Buylla, A., and Berger, M.S. 2005. Neural stem cells and the origin of gliomas. $N$ Engl J Med 353(8): 811-822.

Sanai, N., Tramontin, A.D., Quinones-Hinojosa, A., Barbaro, N.M., Gupta, N., Kunwar, S., Lawton, M.T., McDermott, M.W., Parsa, A.T., Manuel-Garcia Verdugo, J. et al. 2004. Unique astrocyte ribbon in adult human brain contains neural stem cells but lacks chain migration. Nature 427(6976): 740-744.

Sancak, Y., Thoreen, C.C., Peterson, T.R., Lindquist, R.A., Kang, S.A., Spooner, E., Carr, S.A., and Sabatini, D.M. 2007. PRAS40 is an insulin-regulated inhibitor of the mTORC1 protein kinase. Mol Cell 25(6): 903-915.

Sansal, I. and Sellers, W.R. 2004. The biology and clinical relevance of the PTEN tumor suppressor pathway. J Clin Oncol 22(14): 2954-2963.

Sarbassov, D.D., Ali, S.M., Sengupta, S., Sheen, J.H., Hsu, P.P., Bagley, A.F., Markhard, A.L., and Sabatini, D.M. 2006. Prolonged rapamycin treatment inhibits mTORC2 assembly and Akt/PKB. Mol Cell 22(2): 159-168.

Sarbassov, D.D., Guertin, D.A., Ali, S.M., and Sabatini, D.M. 2005. Phosphorylation and regulation of Akt/PKB by the rictor-mTOR complex. Science 307(5712): 10981101. 
Sauvageot, C.M. and Stiles, C.D. 2002. Molecular mechanisms controlling cortical gliogenesis. Curr Opin Neurobiol 12(3): 244-249.

Schuller, U., Heine, V.M., Mao, J., Kho, A.T., Dillon, A.K., Han, Y.G., Huillard, E., Sun, T., Ligon, A.H., Qian, Y. et al. 2008. Acquisition of granule neuron precursor identity is a critical determinant of progenitor cell competence to form Shh-induced medulloblastoma. Cancer Cell 14(2): 123-134.

Sellick, G.S., Barker, K.T., Stolte-Dijkstra, I., Fleischmann, C., Coleman, R.J., Garrett, C., Gloyn, A.L., Edghill, E.L., Hattersley, A.T., Wellauer, P.K. et al. 2004. Mutations in PTF1A cause pancreatic and cerebellar agenesis. Nat Genet 36(12): 1301-1305.

Sengupta, S., Peterson, T.R., and Sabatini, D.M. 2010. Regulation of the mTOR complex 1 pathway by nutrients, growth factors, and stress. Mol Cell 40(2): 310-322.

Serrano, M., Lin, A.W., McCurrach, M.E., Beach, D., and Lowe, S.W. 1997. Oncogenic ras provokes premature cell senescence associated with accumulation of p53 and p16INK4a. Cell 88(5): 593-602.

Shah, O.J. and Hunter, T. 2006. Turnover of the active fraction of IRS1 involves raptormTOR- and S6K1-dependent serine phosphorylation in cell culture models of tuberous sclerosis. Mol Cell Biol 26(17): 6425-6434.

Shaw, R.J. and Cantley, L.C. 2006. Ras, PI(3)K and mTOR signalling controls tumour cell growth. Nature 441(7092): 424-430.

Shen, Q., Wang, Y., Kokovay, E., Lin, G., Chuang, S.M., Goderie, S.K., Roysam, B., and Temple, S. 2008. Adult SVZ stem cells lie in a vascular niche: a quantitative analysis of niche cell-cell interactions. Cell Stem Cell 3(3): 289-300.

Singh, S.K., Clarke, I.D., Terasaki, M., Bonn, V.E., Hawkins, C., Squire, J., and Dirks, P.B. 2003. Identification of a cancer stem cell in human brain tumors. Cancer Res 63(18): 5821-5828.

Singh, S.K., Hawkins, C., Clarke, I.D., Squire, J.A., Bayani, J., Hide, T., Henkelman, R.M., Cusimano, M.D., and Dirks, P.B. 2004. Identification of human brain tumour initiating cells. Nature 432(7015): 396-401.

Soriano, P. 1999. Generalized lacZ expression with the ROSA26 Cre reporter strain. Nat Genet 21(1): 70-71.

Srinivas, S., Watanabe, T., Lin, C.S., William, C.M., Tanabe, Y., Jessell, T.M., and Costantini, F. 2001. Cre reporter strains produced by targeted insertion of EYFP and ECFP into the ROSA26 locus. BMC Dev Biol 1: 4. 
Steck, P.A., Pershouse, M.A., Jasser, S.A., Yung, W.K., Lin, H., Ligon, A.H., Langford, L.A., Baumgard, M.L., Hattier, T., Davis, T. et al. 1997. Identification of a candidate tumour suppressor gene, MMAC1, at chromosome 10q23.3 that is mutated in multiple advanced cancers. Nat Genet 15(4): 356-362.

Subramanian, K.K., Jia, Y., Zhu, D., Simms, B.T., Jo, H., Hattori, H., You, J., Mizgerd, J.P., and Luo, H.R. 2007. Tumor suppressor PTEN is a physiologic suppressor of chemoattractant-mediated neutrophil functions. Blood 109(9): 4028-4037.

Suter, U. and Scherer, S.S. 2003. Disease mechanisms in inherited neuropathies. Nat Rev Neurosci 4(9): 714-726.

Suzuki, A., de la Pompa, J.L., Stambolic, V., Elia, A.J., Sasaki, T., del Barco Barrantes, I., Ho, A., Wakeham, A., Itie, A., Khoo, W. et al. 1998. High cancer susceptibility and embryonic lethality associated with mutation of the PTEN tumor suppressor gene in mice. Curr Biol 8(21): 1169-1178.

Suzuki, A., Nakano, T., Mak, T.W., and Sasaki, T. 2008. Portrait of PTEN: messages from mutant mice. Cancer Sci 99(2): 209-213.

Suzuki, A., Yamaguchi, M.T., Ohteki, T., Sasaki, T., Kaisho, T., Kimura, Y., Yoshida, R., Wakeham, A., Higuchi, T., Fukumoto, M. et al. 2001. T cell-specific loss of Pten leads to defects in central and peripheral tolerance. Immunity 14(5): 523-534.

Tang, M., Iijima, M., Kamimura, Y., Chen, L., Long, Y., and Devreotes, P. 2011. Disruption of PKB signaling restores polarity to cells lacking tumor suppressor PTEN. Mol Biol Cell 22(4): 437-447.

Tavazoie, M., Van der Veken, L., Silva-Vargas, V., Louissaint, M., Colonna, L., Zaidi, B., Garcia-Verdugo, J.M., and Doetsch, F. 2008. A specialized vascular niche for adult neural stem cells. Cell Stem Cell 3(3): 279-288.

Tavazoie, S.F., Alvarez, V.A., Ridenour, D.A., Kwiatkowski, D.J., and Sabatini, B.L. 2005. Regulation of neuronal morphology and function by the tumor suppressors Tsc1 and Tsc2. Nat Neurosci 8(12): 1727-1734.

Taylor, M.D., Liu, L., Raffel, C., Hui, C.C., Mainprize, T.G., Zhang, X., Agatep, R., Chiappa, S., Gao, L., Lowrance, A. et al. 2002. Mutations in SUFU predispose to medulloblastoma. Nat Genet 31(3): 306-310.

TCGA (The Cancer Genome Atlas Research Network). 2008. Comprehensive genomic characterization defines human glioblastoma genes and core pathways. Nature 455(7216): 1061-1068.

Thompson, M.C., Fuller, C., Hogg, T.L., Dalton, J., Finkelstein, D., Lau, C.C., Chintagumpala, M., Adesina, A., Ashley, D.M., Kellie, S.J. et al. 2006. Genomics identifies medulloblastoma subgroups that are enriched for specific genetic alterations. J Clin Oncol 24(12): 1924-1931. 
Tomasiewicz, H., Ono, K., Yee, D., Thompson, C., Goridis, C., Rutishauser, U., and Magnuson, T. 1993. Genetic deletion of a neural cell adhesion molecule variant (N-CAM-180) produces distinct defects in the central nervous system. Neuron 11(6): 1163-1174.

Trotter, J., Karram, K., and Nishiyama, A. 2009. NG2 cells: properties, progeny and origin. Brain Res Rev 63(1-2): 72-82.

Vander Haar, E., Lee, S.I., Bandhakavi, S., Griffin, T.J., and Kim, D.H. 2007. Insulin signalling to mTOR mediated by the Akt/PKB substrate PRAS40. Nat Cell Biol 9(3): 316-323.

Vazquez, F., Grossman, S.R., Takahashi, Y., Rokas, M.V., Nakamura, N., and Sellers, W.R. 2001. Phosphorylation of the PTEN tail acts as an inhibitory switch by preventing its recruitment into a protein complex. J Biol Chem 276(52): 4862748630 .

Voskoglou-Nomikos, T., Pater, J.L., and Seymour, L. 2003. Clinical predictive value of the in vitro cell line, human xenograft, and mouse allograft preclinical cancer models. Clin Cancer Res 9(11): 4227-4239.

Wallace, V.A. 1999. Purkinje-cell-derived Sonic hedgehog regulates granule neuron precursor cell proliferation in the developing mouse cerebellum. Curr Biol 9(8): 445-448.

Wang, V.Y. and Zoghbi, H.Y. 2001. Genetic regulation of cerebellar development. Nat Rev Neurosci 2(7): 484-491.

Ward, R.J. and Dirks, P.B. 2007. Cancer stem cells: at the headwaters of tumor development. Аnпu Rev Pathol 2: 175-189.

Way, S.W., McKenna, J., 3rd, Mietzsch, U., Reith, R.M., Wu, H.C., and Gambello, M.J. 2009. Loss of Tsc2 in radial glia models the brain pathology of tuberous sclerosis complex in the mouse. Hum Mol Genet 18(7): 1252-1265.

Wechsler-Reya, R.J. and Scott, M.P. 1999. Control of neuronal precursor proliferation in the cerebellum by Sonic Hedgehog. Neuron 22(1): 103-114.

Wen, S., Stolarov, J., Myers, M.P., Su, J.D., Wigler, M.H., Tonks, N.K., and Durden, D.L. 2001. PTEN controls tumor-induced angiogenesis. Proc Natl Acad Sci U S A 98(8): 4622-4627.

Wetmore, C., Eberhart, D.E., and Curran, T. 2000. The normal patched allele is expressed in medulloblastomas from mice with heterozygous germ-line mutation of patched. Cancer Res 60(8): 2239-2246. 
Wildonger, J., Jan, L.Y., and Jan, Y.N. 2008. The Tsc1-Tsc2 complex influences neuronal polarity by modulating TORC1 activity and SAD levels. Genes Dev 22(18): 2447-2453.

Yang, Z.J., Ellis, T., Markant, S.L., Read, T.A., Kessler, J.D., Bourboulas, M., Schuller, U., Machold, R., Fishell, G., Rowitch, D.H. et al. 2008. Medulloblastoma can be initiated by deletion of Patched in lineage-restricted progenitors or stem cells. Cancer Cell 14(2): 135-145.

Yilmaz, O.H., Valdez, R., Theisen, B.K., Guo, W., Ferguson, D.O., Wu, H., and Morrison, S.J. 2006. Pten dependence distinguishes haematopoietic stem cells from leukaemia-initiating cells. Nature 441(7092): 475-482.

Yue, Q., Groszer, M., Gil, J.S., Berk, A.J., Messing, A., Wu, H., and Liu, X. 2005. PTEN deletion in Bergmann glia leads to premature differentiation and affects laminar organization. Development 132(14): 3281-3291.

Zhang, J., Grindley, J.C., Yin, T., Jayasinghe, S., He, X.C., Ross, J.T., Haug, J.S., Rupp, D., Porter-Westpfahl, K.S., Wiedemann, L.M. et al. 2006. PTEN maintains haematopoietic stem cells and acts in lineage choice and leukaemia prevention. Nature 441(7092): 518-522.

Zhang, L. and Goldman, J.E. 1996. Generation of cerebellar interneurons from dividing progenitors in white matter. Neuron 16(1): 47-54.

Zheng, H., Ying, H., Yan, H., Kimmelman, A.C., Hiller, D.J., Chen, A.J., Perry, S.R., Tonon, G., Chu, G.C., Ding, Z. et al. 2008. p53 and Pten control neural and glioma stem/progenitor cell renewal and differentiation. Nature 455(7216): 1129-1133.

Zhong, H., Chiles, K., Feldser, D., Laughner, E., Hanrahan, C., Georgescu, M.M., Simons, J.W., and Semenza, G.L. 2000. Modulation of hypoxia-inducible factor lalpha expression by the epidermal growth factor/phosphatidylinositol 3kinase/PTEN/AKT/FRAP pathway in human prostate cancer cells: implications for tumor angiogenesis and therapeutics. Cancer Res 60(6): 1541-1545.

Zhou, X.P., Marsh, D.J., Morrison, C.D., Chaudhury, A.R., Maxwell, M., Reifenberger, G., and Eng, C. 2003. Germline inactivation of PTEN and dysregulation of the phosphoinositol-3-kinase/Akt pathway cause human Lhermitte-Duclos disease in adults. Am J Hum Genet 73(5): 1191-1198.

Zhu, Y., Harada, T., Liu, L., Lush, M.E., Guignard, F., Harada, C., Burns, D.K., Bajenaru, M.L., Gutmann, D.H., and Parada, L.F. 2005. Inactivation of NF1 in CNS causes increased glial progenitor proliferation and optic glioma formation. Development 132(24): 5577-5588. 
Zhu, Y., Romero, M.I., Ghosh, P., Ye, Z., Charnay, P., Rushing, E.J., Marth, J.D., and Parada, L.F. 2001. Ablation of NF1 function in neurons induces abnormal development of cerebral cortex and reactive gliosis in the brain. Genes Dev 15(7): 859-876.

Zimmerman, L., Parr, B., Lendahl, U., Cunningham, M., McKay, R., Gavin, B., Mann, J., Vassileva, G., and McMahon, A. 1994. Independent regulatory elements in the nestin gene direct transgene expression to neural stem cells or muscle precursors. Neuron 12(1): 11-24.

Zindy, F., Nilsson, L.M., Nguyen, L., Meunier, C., Smeyne, R.J., Rehg, J.E., Eberhart, C., Sherr, C.J., and Roussel, M.F. 2003. Hemangiosarcomas, medulloblastomas, and other tumors in Ink4c/p53-null mice. Cancer Res 63(17): 5420-5427.

Zindy, F., Uziel, T., Ayrault, O., Calabrese, C., Valentine, M., Rehg, J.E., Gilbertson, R.J., Sherr, C.J., and Roussel, M.F. 2007. Genetic alterations in mouse medulloblastomas and generation of tumors de novo from primary cerebellar granule neuron precursors. Cancer Res 67(6): 2676-2684.

Zurawel, R.H., Chiappa, S.A., Allen, C., and Raffel, C. 1998. Sporadic medulloblastomas contain oncogenic beta-catenin mutations. Cancer Res 58(5): 896-899. 


\section{APPENDIX. SUPPLEMENTARY DATA}

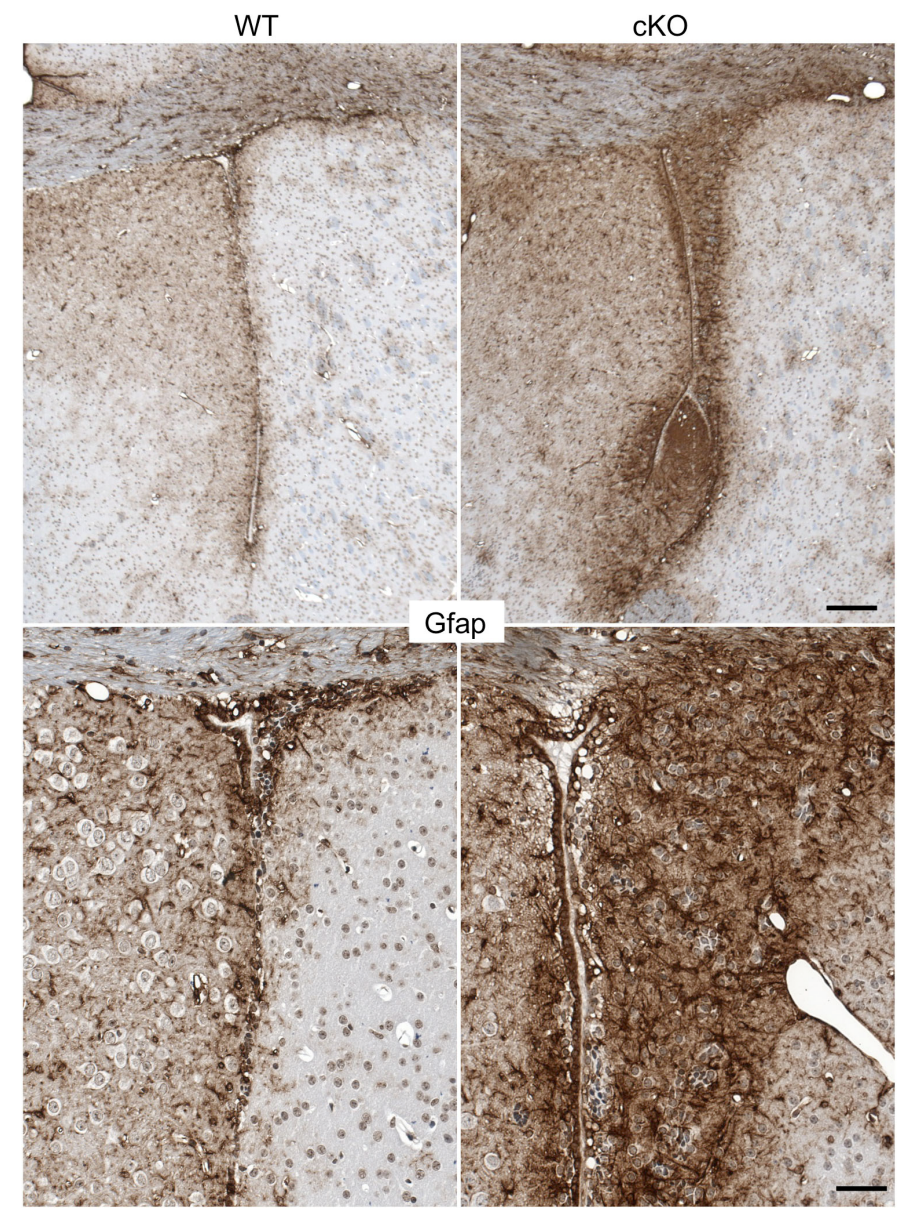

Figure A-1. Increased number and size of astrocytes in the expanded Pten ${ }^{\text {cKo }} \mathrm{SVZ}$. Representative Gfap IHC staining of the SVZ from matched coronal brain sections of Nestin-CerER ${ }^{T 2}$;Pten ${ }^{\text {loxP/loxP }}$ (cKO) mice injected with TM on P30-32 and analyzed at 6 months of age shows increase number and size of astrocytes in the expanded cKO SVZ. Bottom panels are the dorsal part of the SVZ. Scale bar: top panels $=200 \mu \mathrm{m}$, bottom panels $=50 \mu \mathrm{m}$. 


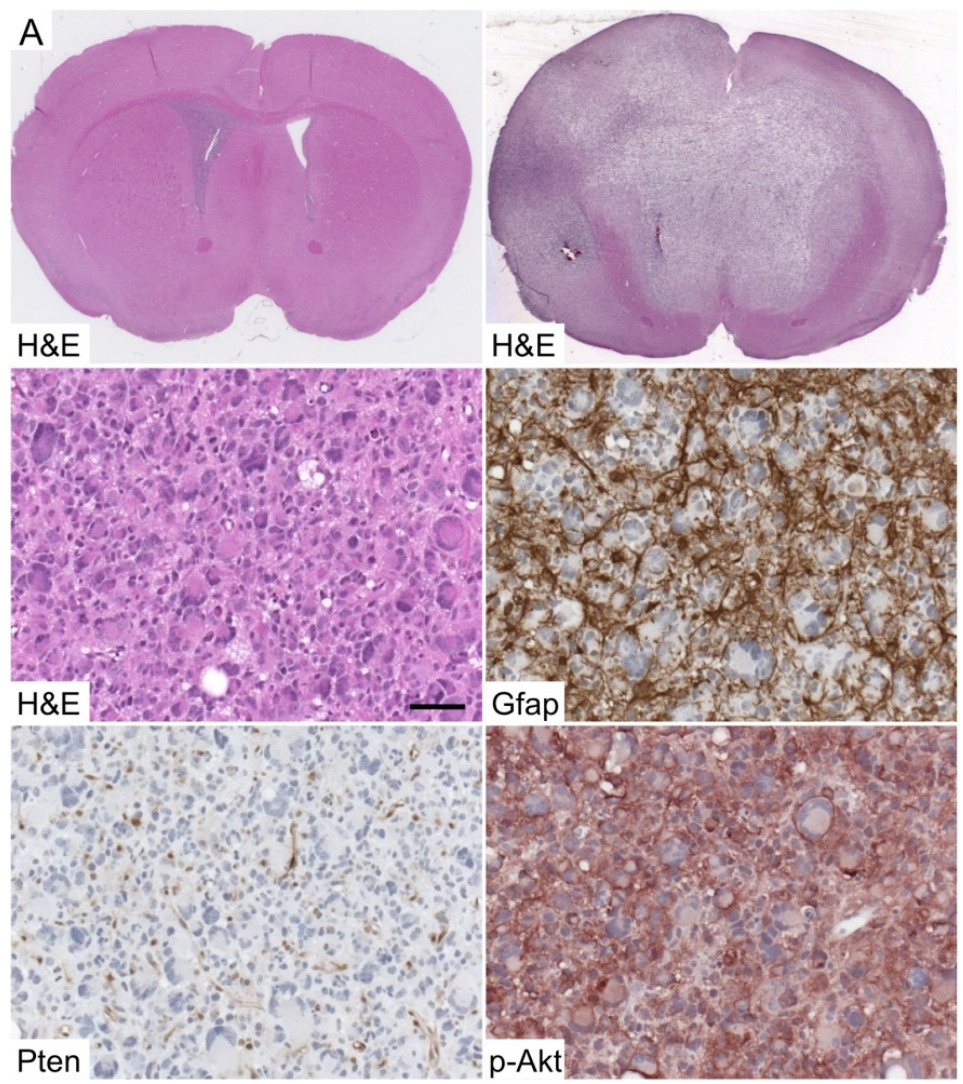

B Survival of Pten; Trp53 (adult induction):Survival proportions

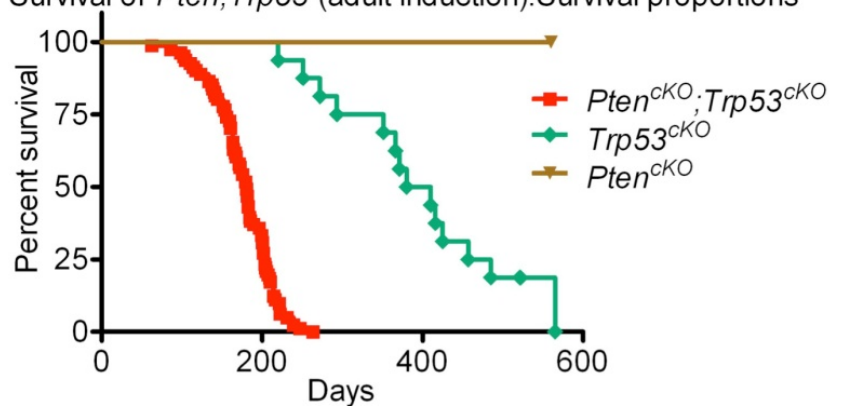

Figure A-2. Deletion of Pten synergizes with Trp53 mutations to drive high-grade gliomas in adult brain arising from the SVZ.

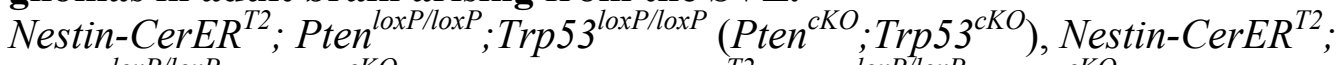
Trp53 $3^{\text {loxP/loxP }}\left(\operatorname{Trp}^{\text {Tr }} 3^{\text {cKO}}\right)$ and Nestin-CerER ${ }^{T 2}$ Pten $^{\text {loxPlloxP }}\left(\right.$ Pten $\left.^{\text {cKO }}\right)$ mice were induced with TM on P28-30. (A) Top panels: H\&E staining of representative high-grade gliomas arising from the SVZ. Middle and bottom panels: representative H\&E, Gfap, Pten and pAkt IHC staining of the high-grade gliomas from the SVZ show the tumor is Gfap+, Pten- and p-Akt+. Scale bar: $50 \mu \mathrm{m}$. (B) Kaplan-Meier survival curves show $P t e n^{c K O} ; \operatorname{Trp}^{3^{c K O}}$ mice with a median tumor onset of 180 days of age (red curve,) and $\operatorname{Trp} 53^{c K O}$ with a median morbidity age of 395 days (green curve). The Pten ${ }^{c K O} ; \operatorname{Trp}^{\text {c }} 3^{\text {cKO }}$ mice developed high-grade gliomas with $50 \%$ of penetrance as well as peripheral tumors The majority of $\operatorname{Trp} 53^{c K O}$ were euthanized because of peripheral tumors. 

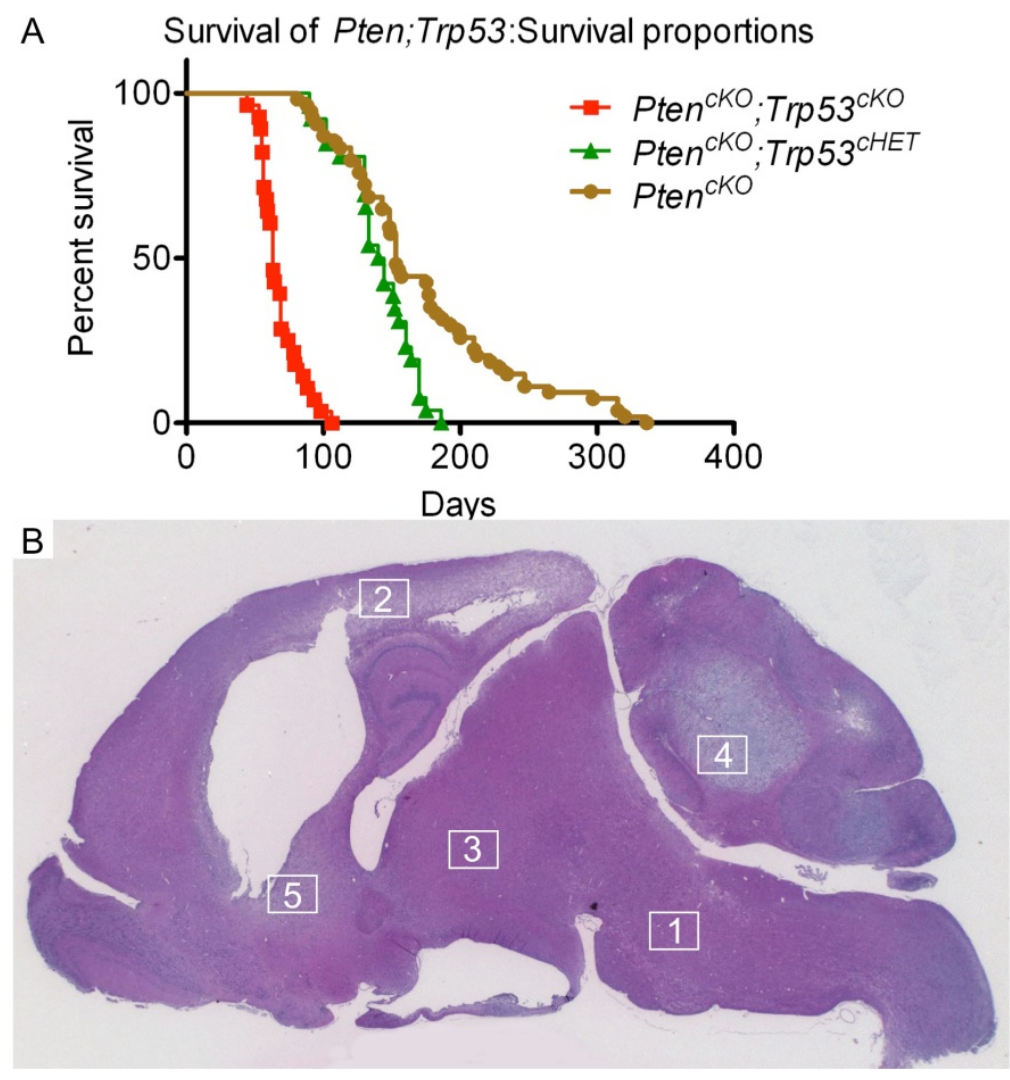

Figure A-3. Nestin-CreER $R^{T 2} ;$ Pten $^{c K o} ; T p 53^{c H E T}$ mice developed highly infiltrative gliomas throughout brain.

Nestin-CerER ${ }^{T 2} ;$ Pten $^{\text {loxP/loxP }} ; \operatorname{Trp}^{\text {loxP/loxP }}\left(\right.$ Pten $\left.^{\text {cKO }} ; \operatorname{Trp}^{\text {TrKO }}{ }^{\text {cKO }}\right)$, Nestin-

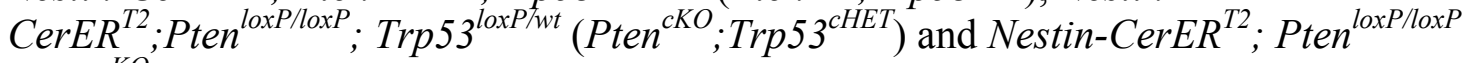
(Pten $^{c K O}$ ) were induced with TM on P0-1. (A) Kaplan-Meier survival curves show $P_{t e n}{ }^{c K O} ; \operatorname{Trp} 53^{\text {cHET }}$ mice with a median survival of 142 days of age (green curve) compared to 63 days for Pten ${ }^{c K O} ; \operatorname{Trp}_{53} 3^{c K O}$ mice (red curve which developed medulloblatomas) and 153 days for $P t e n{ }^{c K O}$ mice (brown curve which were euthanized due to macrocephaly and hydrocephaly) (see also Chapter 3). (B) Pten ${ }^{c K O} ; \operatorname{Trp} 53^{c H E T}$ mice developed highly infiltrative gliomas throughout brain. White boxes indicate the regions of interest shown in Figure A-4 with high magnification. 1: brain stem; 2: corpus callosum and cortex; 3: thalamus; 4: cerebellum; 5: SVZ. 


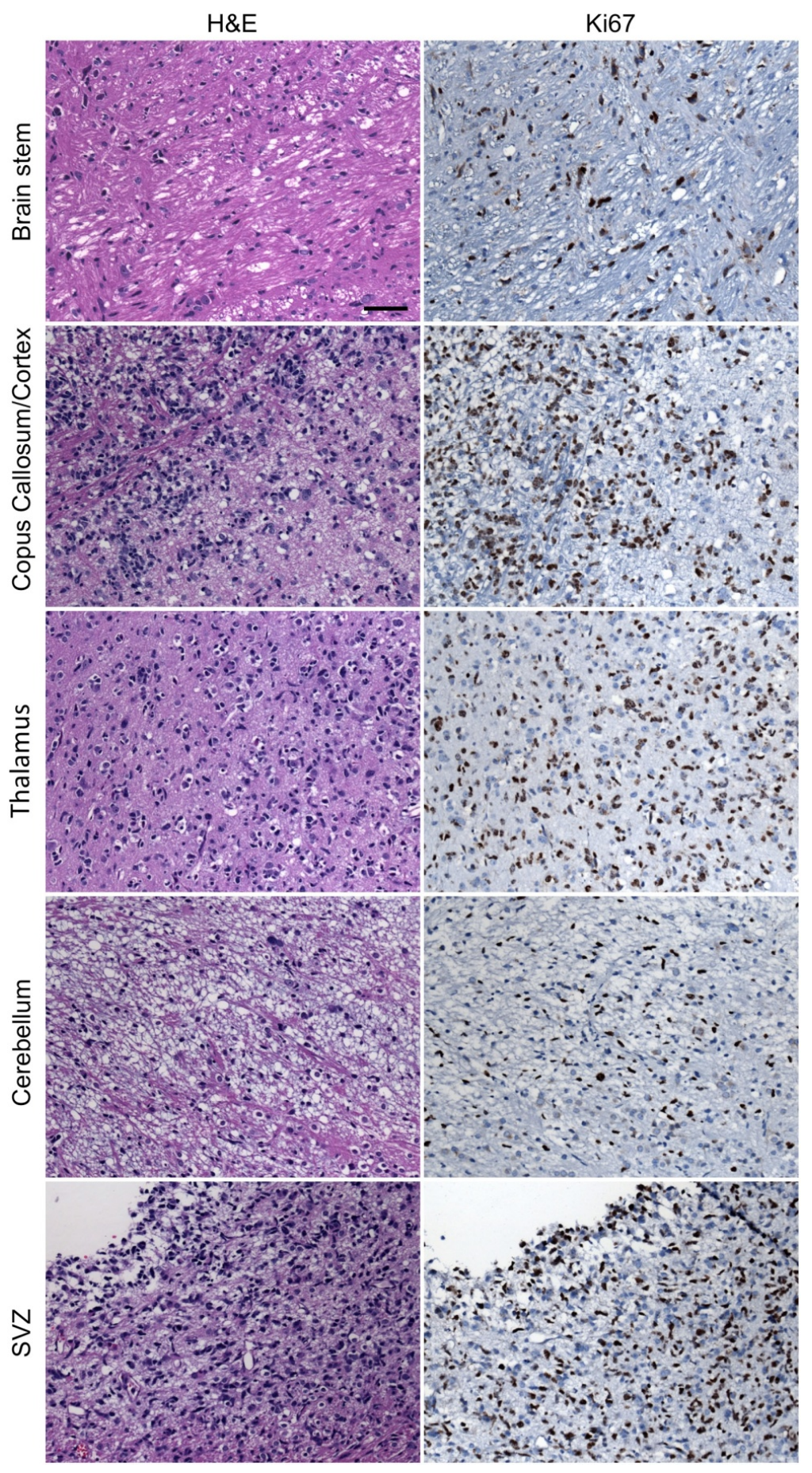

Figure A-4. Highly infiltrative gliomas developed in Nestin-CreER ${ }^{T 2}$ Pten $^{\text {cKo }}$; Tp53 $3^{\text {cHET }}$ mice.

Representative H\&E and Ki67 IHC staining of infiltrative gliomas developed in the

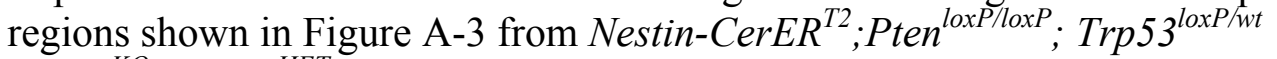

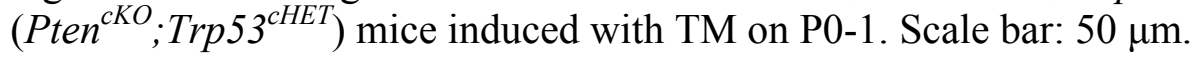




\section{Supplementary movie A-1. WT mice.}

Supplementary movie A-2. Pten ${ }^{\text {cKo }}$ mice.

A Cre expression construct was transfected by in vivo electroporation at $\mathrm{P} 2$ into the SVZ of WT or Pten ${ }^{\text {floxflox }}$ (cKO) mice carrying a ROSA-lox-STOP-lox-EYFP allele. Acute brain slices were prepared at P19-20, and cells in which Cre-mediated recombination occurred were visualized by EYFP reporter expression using two-photon microscopy. Images were captured every 5 minutes for 2.5 hours, and are presented in a movie of 4 seconds. Movies show the z-stack projections of 20 consecutive optical planes of section. 


\section{VITA}

Guo Zhu was born in Hunan Province, China in 1978. He graduated from the Xiangya School of Medicine at the Central South University with a Bachelor of Medicine in Medicine in 2002 and a Master of Science in Pathology and Pathophysiology in 2005. After moved to the United States in 2005, he enrolled in the Integrated Program in Biomedical Sciences at the University of Tennessee Health Science Center for graduate study. In June 2006, he joined Dr. Suzanne Baker's laboratory at the St. Jude Children's Research Hospital and conducted research in Pten function in the brain development and tumorigenesis. He is expected to receive his Doctor of Philosophy degree from the University of Tennessee in May 2011. 